\title{
IMAGING 3D TISSUE FIBER ORGANIZATION USING OPTICAL POLARIZATION TRACTOGRAPHY
}

\author{
A Dissertation presented to
}

the Faculty of the Graduate School at the University of Missouri

\author{
In Partial Fulfillment
}

of the Requirement for the Degree

Doctor of Philosophy

by

YUANBO WANG

Dr. Gang Yao, Dissertation Supervisor

JULY 2017 
The undersigned, appointed by the dean of the Graduate School, have examined the dissertation entitled

\section{IMAGING 3D TISSUE FIBER ORGANIZATION USING OPTICAL POLARIZATION TRACTOGRAPHY}

presented by Yuanbo Wang,

a candidate for the degree of Doctor of Philosophy,

and hereby certify that, in their opinion, it is worthy of acceptance.

Professor Gang Yao

Department of Bioengineering

Professor Dongsheng Duan

Department of Molecular Microbiology \& Immunology

Professor Shinghua Ding

Department of Bioengineering

Professor Jinglu Tan

Department of Bioengineering 


\section{ACKNOWLEDGEMENTS}

First of all, I would like to express my most sincere gratitude to my advisor Dr. Gang Yao, for guiding me and training me to become a good researcher. Without his help, guidance, and encouragement in the past five years, I would not be able to complete my dissertation research successfully. I am indebted to Dr. Dongsheng Duan for all his great support and constructive advice which are essential to completing my research projects. I also sincerely appreciate my other doctoral committee members, Drs. Shinghua Ding and Jinglu Tan, for their time and guidance throughout my study.

I would like to thank my current and former labmates, Dr. Chuanmao Fan, Dr. Randima Dinalankara, Mr. Ravanfar Mohammadreza, Ms. Azinfar Leila, Ms. Xuan Yao, and Dr. Chathuri Daluwatte. Their warm help and friendship are invaluable. In addition, I am thankful for Keqing Zhang, Wasala Nalinda, Hakim Chady, and Yongping Yue, for their great help, support, and valuable discussions in many of my research projects.

Finally, I would like to show my particular appreciation to my parents and my wife, for their unconditional support, care, and encouragement. 


\section{Table of Contents}

ACKNOWLEDGEMENTS ................................................................................................ ii

List of Figures................................................................................................................. $\mathrm{v}$

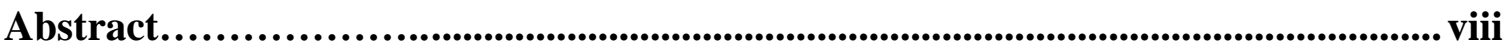

Chapter 1. Tissue birefringence imaging ................................................................................ 1

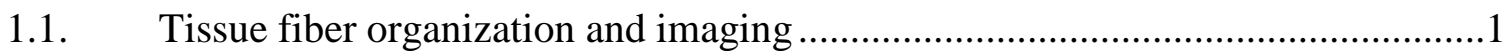

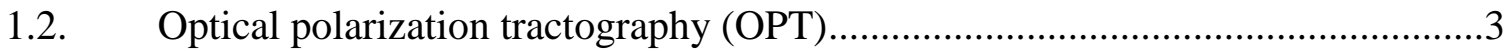

1.3. Imaging fiber structural changes in animal tissues of Duchene muscular

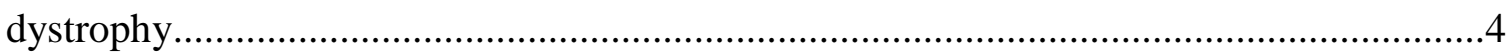

1.4. Outline of this dissertation research .........................................................6

Chapter 2. Histology validation of mapping depth-resolved fiber orientation using OPT ......................................................................................................... 8

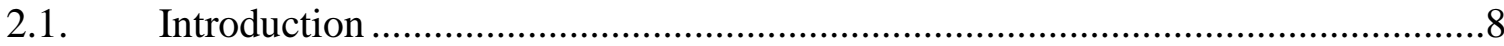

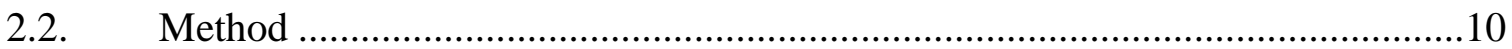

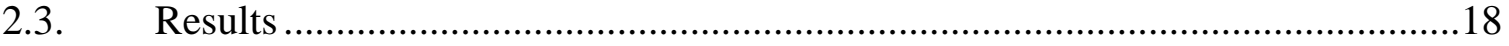

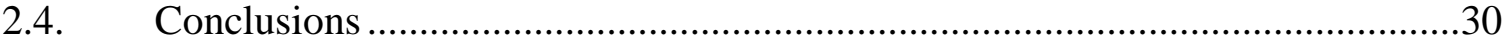

Chapter 3. Tractographic imaging of the mouse heart using OPT ........................... 32

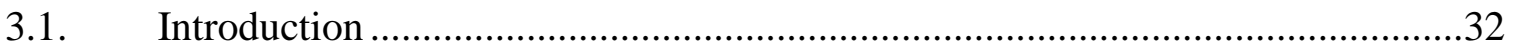

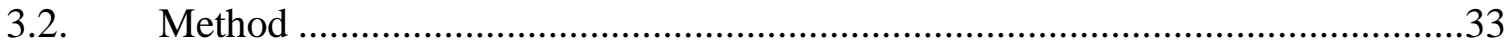

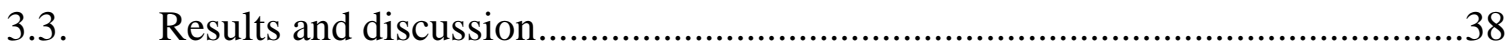

3.4. Conclusion................................................................................................

Chapter 4. OPT imaging of heart structural remodeling in a mouse model of Duchenne cardiomyopathy ................................................................................. 44

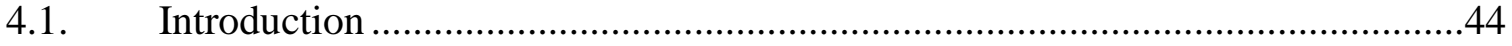

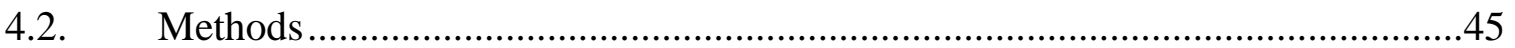

4.3. Results \& Discussion ................................................................................

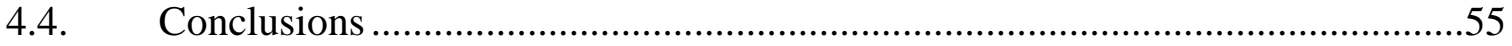


Chapter 5. Imaging microscopic cardiac damage in mdx mice using OPT.............. 56

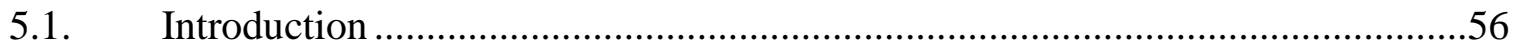

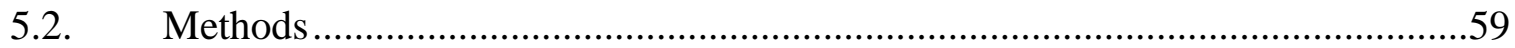

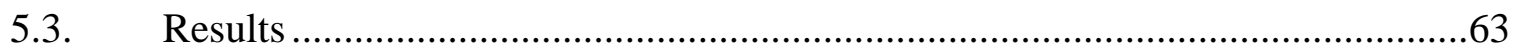

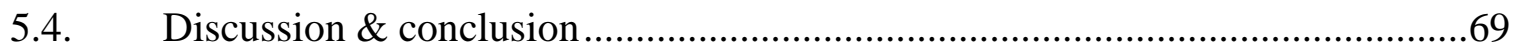

Chapter 6. OPT revealed significant fiber disarray in ex vivo skeletal muscles of mdx mice .......................................................................................................... 71

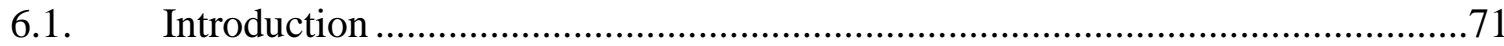

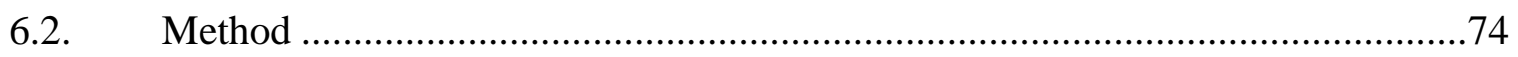

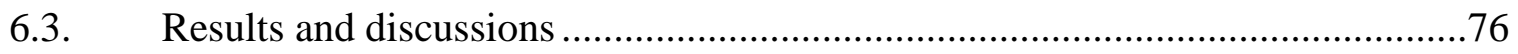

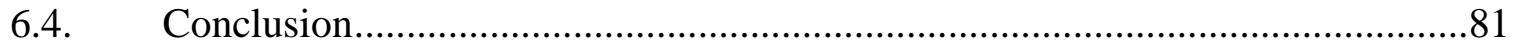

Chapter 7. Dual-angle OPT imaging of absolute 3D fiber orientation in tissue .... 83

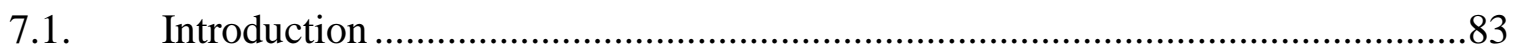

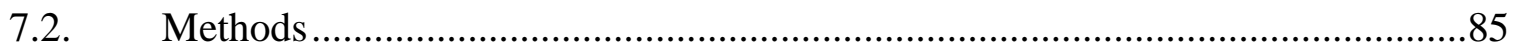

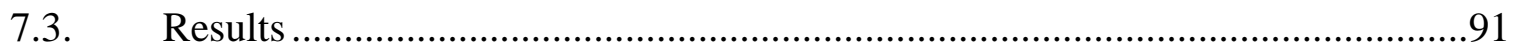

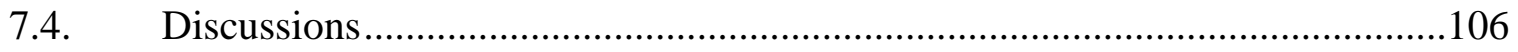

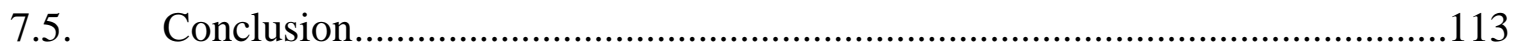

Chapter 8. Summary and future direction ...................................................................... 114

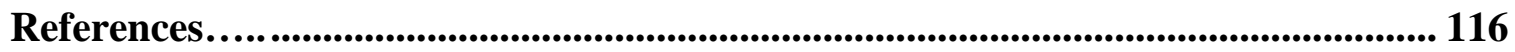

VITA 


\section{List of Figures}

Figures

Page

Figure 2-1. An illustration of image distortion induced by surface refraction.............. 15

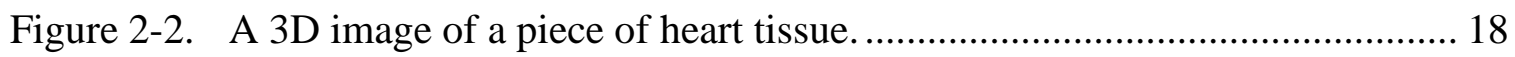

Figure 2-3. An example of the surface refraction correction..................................... 20

Figure 2-4. A direct comparison of the depth-resolved myocardiac fiber orientation measured from histology and OPT ................................................. 22

Figure 2-5. The correlation results between OPT and histology................................. 24

Figure 2-6. An example comparison of tractography computed using OPT and histology at depths of $0.35 \mathrm{~mm}$ and $0.65 \mathrm{~mm}$ from the epicardium in a heart tissue

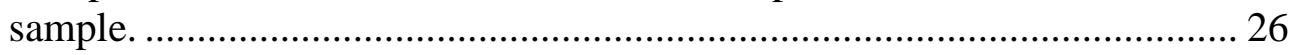

Figure 2-7. Analysis of possible sources of error..................................................... 28

Figure 2-8. A comparison of the (a) histology tractography and (b) OPT in a tissue sample with significant amount of orientation changes and tissue deformation caused by histology processing. ........................................ 29

Figure 3-1. An illustration of the imaging geometry................................................. 35

Figure 3-2. An illustration of the procedure for reconstructing the tractography in a

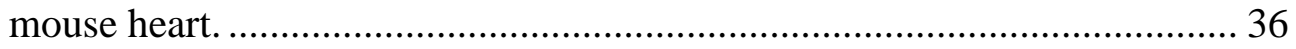

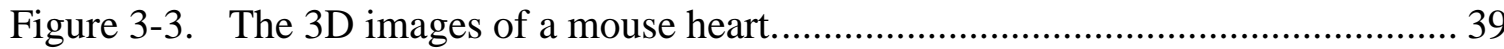

Figure 3-4. An analysis of the depth dependent fiber orientation changes from selected ROIs...................................................................................... 40

Figure 4-1. 3D OPT images of whole mouse hearts........................................... 48

Figure 4-2. (a) An illustration of the "window" in LV and RV (blue boxes) for quantitative analysis. 50

Figure 4-3. (a) Examples of color-coded "slopes" of fiber orientation change with depth in the LV and RV of the same hearts shown in Fig. 4-2. 52

Figure 4-4. A group comparison of the average rate of fiber orientation change with depth in the LV and RV of the six BL6 mice and six mdx mice................ 54

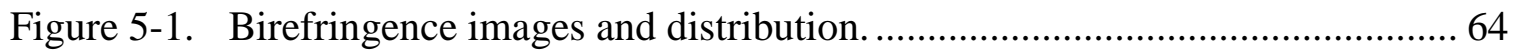


Figure 5-2. 2D images of (a) entropy, (b) circular standard deviation $\Delta \theta$, and (c) segmented tissue damage at $300 \mu \mathrm{m}$ depth from the same hearts shown in Fig. 5-1.

Figure 5-3. Distributions of circular standard deviation $\Delta \theta$ (a) and entropy (b) computed in the whole heart from the surface to $0.5 \mathrm{~mm}$ in depth. 65

Figure 5-4. A comparison between OPT and histology (HE) sectioned along the long axis of an mdx heart.

Figure 5-5. A comparison between OPT and histology (HE) sectioned along the short axis of an mdx heart.

Figure 6-1. Representative images of (a) OCT intensity, conventional cumulative (b) retardance and (c) optic axis of the mdx 4cv TA muscle.

Figure 6-2. The corresponding images of local (a) retardance and (b) optic axis at the same imaging locations as in Fig. 6-1

Figure 6-3. (a, b) The 3D OPT images of two TA muscles from two different mdx4cv mice......

Figure 7-1. An illustration of the OPT measurement geometry.................................. 87

Figure 7-2. Single-scan OPT and registration of two image volumes acquired in dualangle OPT.

Figure 7-3. 3D dual-angle OPT and registration of multiple image volumes obtained at different 3D positions. .............................................................................. 94

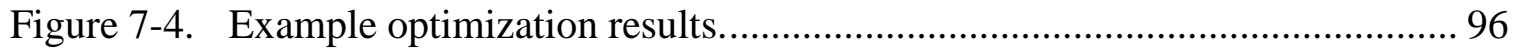

Figure 7-5. The correlation between measured and expected projection angles in fifty randomly selected ROIs $\left(48 \times 48 \times 48 \mu \mathrm{m}^{3}\right)$ from the EDL muscle. .............. 97

Figure 7-6. Dual-angle OPT of a piece of the mouse tibialis anterior (TA) muscle. ..... 99

Figure 7-7. A comparison of the fiber organization obtained in dual-angle OPT with that obtained by analyzing OCT intensity profiles in the en face plane at depths from $200 \mu \mathrm{m}$ to $800 \mu \mathrm{m}$. 101

Figure 7-8. 3D visualization of a piece of the bovine cartilage in (a) intensity, (b) 3D fiber tractography from dual-angle measurements, and (c) the 2D tractography by projecting the $3 \mathrm{D}$ fibers shown in (b) to the $\mathrm{AB}-, \mathrm{AC}-$, and BC-plane as labeled in (a). 102

Figure 7-9. (a) The en face intensity and tractography images obtained from direct OPT imaging of the side of the cartilage sample (the "side-scan" as labeled in Fig. 7-8). 104 
Figure 7-10. (a) The fiber orientation over the depth obtained from the side-scan image by averaging all $\mathrm{B}$-scan inside a $0.5 \mathrm{~mm}$ wide ROI. 


\section{Abstract}

Optical polarization tractography (OPT) is a new imaging technology developed based on an advanced Jones matrix implementation of polarization-sensitive optical coherence tomography (PSOCT). OPT can acquire high-resolution, three-dimensional (3D), depthresolved images of fiber organization in tissue. To validate OPT's accuracy in measuring fiber orientation, a comprehensive histology comparison study was conducted using heart tissues which are known to have a depth-dependent fiber orientation change. A systematic image processing procedure was developed to register histology images with OPT images so that the pixel-wise difference between the two measurements can be compared in details. The validation results indicated that OPT can reveal the tissue fiber tractography with histology-like resolution.

OPT was then applied to image freshly excised heart samples of the mdx mouse model of Duchene muscular dystrophies (DMD). A rotational imaging platform was developed to obtain OPT images of the excised whole mouse heart. The imaging light was repetitively scanned along the long axis of the heart while the heart was rotated continuously on the imaging platform. The acquired 3D image data were then transformed to construct the 3D whole heart image. The "cross-helical" laminar architecture of the myocardial fibers can be clearly visualized. More importantly, the OPT results revealed significant global and microscopic structural remodeling in the heart of the mdx mouse. 
OPT can also be applied to image fiber organization in other fibrous tissue samples. For example, OPT revealed focal fiber disorganization in the tibialis anterior (TA) muscle of the mdx mouse, which was confirmed in histology as muscle damage. The 3D OPT images of the TA muscle can be quantified by analyzing the randomness of the fiber orientation distribution. A "fiber disarray" index can be computed to automatically identify and visualize all damaged tissues in the 3D TA muscle.

Since the OPT only images the projected fiber orientation within a plane perpendicular to the light propagation, a dual-angle imaging procedure was developed to obtain the absolute 3D fiber orientation. The two 3D OPT images of the same tissue acquired at two different view angles were registered to reconstruct the image of the absolute 3D fiber orientation. This new method was validated by imaging a mouse extensor digitorum longus (EDL) muscle placed at various known positions. The capability of this dual-angle OPT method was demonstrated by visualizing the absolute 3D muscle fiber structure in mouse TA muscles and the unique arcade collagen fiber architecture in a piece of articular cartilage.

In summary, the results presented in this dissertation study indicated that the newly developed OPT technology can obtain high-resolution 3D image of tissue fiber organization. OPT may provide a practical tool for studying disease related fiber structural changes in many fibrous tissues. 


\section{Chapter 1. Tissue birefringence imaging}

\subsection{Tissue fiber organization and imaging}

This dissertation research was focused on developing a new "optical polarization tractography' technology for imaging the fiber organization in fibrous tissues such as muscle, skin, cartilage, tendons, nerve, teeth, etc. The fibrous structures in these tissues play an important role in realizing their corresponding physiological functions and can be altered due to disease or damage. For example, the muscle fibers are very well organized in skeletal muscles. Any abnormal changes in the organization caused by injury or disease would affect the normal force generation (Lovering et al. 2013). The myocardial fibers in the heart form a special "cross-helically" laminar architecture (Streeter Jr et al. 1969). This unique fiber architecture is important to the mechanical function of the heart and it is linked to many diseases including myocardial infarction and heart failure (Benson et al. 2008; Sosnovik et al. 2014). Articular cartilage contains a network of collagen fibril organized into three depth-dependent zones: superficial zone, transitional zone and radial zone (Mittelstaedt et al. 2011). This arcade architecture helps to maintain the structural integrity in response to stress induced by joint loading and motion. Any disruptions to this structure may indicate pathological diseases such as osteoarthritis (Clark 1990; Mittelstaedt et al. 2011).

Due to the importance of fiber organization in many fibrous tissues, an imaging 
techniques that can characterize the fiber structure is potential helpful in basic research and clinic applications. Scanning electron microscope (SEM) with super-high resolutions $(<10 \mathrm{~nm})$, is most advanced in visualization of small fiber units such as collagen fibril with typical diameters between 40nm and 60nm in articular cartilage (Clark 1990). Light microscopy based histology imaging is widely used in investigating general fibrous tissue. Confocal light microscopy further improved the imaging resolution to as high as $\sim 0.1 \mu \mathrm{m}$ (Kim et al. 2009). However, conventional microscopic imaging techniques often require sample processing and sectioning, and hence are time consuming and destructive.

Tissue birefringence is an optical property that exists in fibrous tissues. The optic axis obtained as the part of the birefringence measurements can be served as a way to indicate the fiber orientation in fibrous tissue. Polarized light microscopy (PLM) extends conventional light microscopy by incorporating polarization components to measure the tissue birefringence and fiber orientation. It is commonly used for mapping the orientations of the collagen fibers in cartilage (Xia et al. 2001). However, samples used in PLM are still required to be sectioned.

Alternatively, diffusion-tensor magnetic resonance imaging (DTI) has been established as a state-of-art method for imaging the fiber organization in fibrous tissue noninvasively. DTI was widely used in imaging cardiac fibers in heart (Sosnovik et al. 2009a), nerve fibers in brain (Zhang et al. 2003) and spinal cord (Facon et al. 2005), and muscle fibers in skeletal (Froeling et al. 2014). However, the resolution in DTI imaging is normally limited to submillimeter which is insufficient for thin and small tissues. 


\subsection{Optical polarization tractography (OPT)}

Optical polarization tractography (OPT) is developed to provide a practical tool that can image tissue fiber organization with high-resolution and in a non-destructive way.

OPT is based on polarization-sensitive optical coherence tomography (PSOCT). PSOCT is a functional extension of optical coherence tomography (OCT) (Huang et al. 1991) which is an established non-invasive imaging technique. OCT images the depthresolved tissue structure using the light interferometry. PSOCT uses polarized incident light and polarization-sensitive detection (Hitzenberger et al. 2001) to image tissue birefringence in addition to OCT intensity images.

Conventional PSOCT technology however can only measure the "cumulative" sample properties. For example, the obtained "retardance" is an integrated result from a given position to the sample surface; whereas the computed axis is affected by fiber orientation over the entire light-path and cannot represent the true local fiber orientation. The slope of the conventional cumulative retardance was considered as a simple representation of local retardance in several studies (Pasquesi et al. 2006; Yang et al. 2013). However this approach fails when the optic axis also varied with depth. Additional studies have proposed methods to calculate local polarization properties based on Jones matrix PSOCT (Kemp et al. 2005; Todorović et al. 2008; Makita et al. 2010). However, local optic axis images have not been successfully obtained. A method on correcting cumulative 
optic axis (Fan \& Yao 2010a) was attempted to resolve the axis "jumping" due to retardation accumulation. However, the method is valid only in samples with depthindependent optic axis (Fan \& Yao 2012c) and negligible diattenuation (Fan \& Yao 2013).

The recently developed OPT algorithm successfully derived the comprehensive local polarization properties (Fan \& Yao 2012c, b, a, 2013) based on Jones matrix PSOCT results using advanced Jones calculus. It removed the local diattenuation (imaginary part of the complex retardance) and reconstructed the true local optic axis using an iterative procedure. Because OPT can reveal local fiber orientation, it enabled high-resolution optical tractography for imaging fiber architecture in tissue.

Optical polarization based method only measures the "apparent" birefringence in a projection plane perpendicular to the light propagation. Clearly, the measured orientations do not represent the true $3 \mathrm{D}$ fiber orientation unless the light incidence is perpendicular to the fiber. The $3 \mathrm{D}$ orientation can be obtained by imaging the sample from different angles as reported previously (Ugryumova et al. 2006; Ugryumova et al. 2009). Such variableangle imaging approach can be also used in OPT to derive the depth-resolved 3D fiber orientation as demonstrated in this dissertation (Chapter 7).

\subsection{Imaging fiber structural changes in animal tissues of Duchene muscular dystrophy}

Tissue samples from the mdx model of Duchene muscular dystrophy (DMD) were used in our research to test OPT's imaging capability. DMD affects one in every 3,500 male infants 
(Hoffman et al. 1987). Many DMD patients eventually die from respiratory and cardiac impairment (Klyen et al. 2008). The mdx mice are widely used animal models to study DMD (Radley et al. 2007; Hakim et al. 2011). Several treatment options such as dystrophin gene therapy are under active development (Mercuri \& Muntoni 2013).

Muscle imaging is helpful for studying DMD pathology and understand treatment effects. Light microscope images from sectioned animal histology slices were conventionally used to study DMD (Radley et al. 2008). Noninvasive imaging technique such as diffusion-tensor based magnetic resonance imaging (DTI) has been explored to study DMD (Saupe et al. 2009; McMillan et al. 2011; Ponrartana et al. 2014). Fiber tractography from DTI has been found useful for accessing muscle damage (McMillan et al. 2011). However, the sub millimeter resolution in DTI limits its application to large tissue damages.

OCT has also been used in investigating the skeletal muscle damage (Klyen et al. 2008; Klyen et al. 2011; Lovering et al. 2013; Yang et al. 2014). Muscle necrosis can be identified in OCT intensity images (Klyen et al. 2008; Klyen et al. 2011). Deep muscle imaging were also reported using needle probe (Yang et al. 2014). Optical attenuation coefficient, which was extracted from the depth dependent OCT signal (Scolaro et al. 2012), was found as useful marker for identification of necrotic lesions (Klyen et al. 2014). The weakness of the attenuation coefficient is that it does not contain depth-resolved information unless the local coefficient is computed from segmented depth profiles. However, separating the imaging depth into small potions would degrade the accuracy of 
the extracted attenuation coefficients.

The optical birefringence obtained in PSOCT was found useful in identifying muscle damage. Different "banded gap" on cumulative birefringence have been reported in the exercised mdx muscles (Pasquesi et al. 2006). The slop of cumulative birefringence extracted using linear regression has been reported for identification of muscle necrosis (Yang et al. 2013). Such cumulative measures, however, are also difficult to identify the depth-resolved lesions.

Because OPT can extract depth-resolved local polarization properties with cellularscale resolution (Fan \& Yao 2013; Wang et al. 2014), it has the potential to detect muscle damage with better accuracy. Both skeletal and cardiac tissues from $m d x$ mice were used in this research to investigate and demonstrate OPT's imaging capability.

\subsection{Outline of this dissertation research}

Chapter 2 described a validation study where the depth dependent fiber orientation measured in heart tissue using OPT was compared with that obtained in histology. The OPT results correlated well with histology results in pixel-wise comparisons. Chapter 3 described an imaging method that enabled OPT to obtain fiber tractography in a whole freshly excised mouse heart. The results showed that OPT clearly revealed the doublehelical myocardial fiber architecture in the heart.

Using the method reported in Chapter 3, Chapters 4 and 5 showed that OPT can reveal both global and microscopic myocardial fiber structural changes in a mouse model 
of Duchene muscular dystrophy. Chapter 6 showed that OPT can also image muscle damage in the tibialis anterior (TA) muscle of the mdx mouse. The mdx samples showed regions with clear weaker birefringence and fiber disarray. This segmentation was compared with segmentation from birefringence, and both methods showed similar results. Chapter 7 described an implementation of dual-angle OPT to map the absolute 3D fiber orientations in tissue. The method was first validated using an EDL samples with relatively simple fiber orientation. The method was then validated in TA muscle and the projections from 3D results were compared with the results from OCT intensity. Finally, the dual-angle imaging method successfully constructed the well-known "arcade" collagen fiber structure in articular cartilage. 


\section{Chapter 2. Histology validation of mapping depth-resolved fiber orientation using OPT *}

\subsection{Introduction}

Myofiber organization in cardiac muscle greatly influences the mechanical and electrical function of the heart (Streeter Jr et al. 1969; Taccardi et al. 1994). Action potential propagation is 2.1 to 10 times faster (Kléber \& Rudy 2004) along the longitudinal direction of cardiac myofibers than at the transverse direction. Myofiber disorganization compromises cardiac function. Alterations in cardiac muscle structure have been found as a result of myocardial infarction (Strijkers et al. 2009). Therefore "tractography", an imaging tool that can visualize microstructural details of tissue fiber organization, is valuable for both basic research and clinical diagnosis. Despite its superior spatial resolution, histological assessment is time consuming and limited to imaging of small areas of fixed tissue. Alternatively, diffusion-tensor based magnetic resonance imaging (DTI) (Sosnovik et al. 2009a) has been established as a state-of-art method for imaging the 3D fiber organization in the heart; however, the image resolution in DTI is usually limited to submillimeters. Such resolution is not sufficient for imaging in small animal models which are widely used in cardiovascular research.

\footnotetext{
* This chapter was from a published article: Wang, Y., Zhang, K., Wasala, N.B., Yao, X., Duan, D. and Yao, G., 2014. Histology validation of mapping depth-resolved cardiac fiber orientation in fresh mouse heart using optical polarization tractography. Biomedical optics express, 5(8), pp.2843-2855.
} 
High resolution optical coherence tomography (OCT) has been explored to quantify fiber orientation in heart muscles (Fleming et al. 2008; Ambrosi et al. 2012; Goergen et al. 2012). This intensity-based method requires a high enough spatial resolution to resolve individual muscle fibers. The myocardial fibers in small animals such as mice typically have a diameter less than $3.0 \mu \mathrm{m}$. Although OCT systems with a $\sim 1 \mu \mathrm{m}$ spatial resolution is available, it is challenging to achieve simultaneously a large field of view and depth of view required for imaging in a large tissue e.g. the entire mouse heart.

We recently developed a high-resolution optical tractography method based on Jones matrix optical coherence tomography (JMOCT) (Park et al. 2004; Fan \& Yao 2010b; Makita et al. 2010; Fan \& Yao 2012a). Our technology can derive the depth-resolved local polarization properties including optic axis, retardation, and diattenuation from conventional cumulative results (Fan \& Yao 2012a). Because the local (slow) optic axis is aligned with fiber axis, it can be used to map fiber orientation within the imaging plane (Fan \& Yao 2010a). Measuring fiber orientation from local optic axis has been previously investigated using time-domain Mueller matrix PSOCT (Todorović et al. 2004; Jiao et al. 2005). However, these early studies only obtained fiber orientation at a few discrete depths along a single A-line even with a significant amount of averaging (Todorović et al. 2004; Jiao et al. 2005). Our Jones matrix based method (Fan \& Yao 2012c, b) greatly improved signal-to-noise and enabled tractography in 3D tissues (Fan \& Yao 2013; Wang \& Yao 2013). This new tractography technology inherits the intrinsic advantages of high resolution and fast imaging speed in OCT. It can measure fiber orientation with a single 
pixel resolution without the need of a super-high resolution OCT to resolve individual muscle fibers. For convenience, we refer to this technology for mapping depth-resolved fiber orientation as Optical Polarization Tractography (OPT).

We have demonstrated the capability of OPT in samples with homogeneous optic axis as well as in samples with depth-varying optic axis (Fan \& Yao 2012b). Most recently, we applied this technology for imaging fiber orientation in a bovine heart sample (Fan \& Yao 2013) and a fixed mouse heart (Wang \& Yao 2013). Overall these studies showed good agreement with current knowledge of the myocardial fiber architecture in heart. In this study, we further validated the accuracy of OPT technology by comparing the optical tractography results directly with histology images obtained from the mouse heart. A comprehensive methodology was developed to correct the image distortions in OPT and register the optical tractography with histology images. The results showed that OPT technology can accurately image depth-resolved fiber organization in fresh heart tissues and can reveal microstructural details at the histological level.

\subsection{Method}

\subsubsection{Tissue preparation and histology processing}

A total of seven 2 to 6 -m-old mice were used in this study including four C57BL/10 mice and three mdx mice (Emery \& Muntoni 2003). The mouse heart was excised immediately after euthanasia. A small piece of the heart tissue of roughly $2 \sim 4 \mathrm{~mm}$ in size was cut from 
either the left or right ventricles from each heart. This piece of heart tissue was imaged first using the OPT system. Then the tissue sample was embedded in optimal cutting compound and frozen in liquid nitrogen. For histology processing, the tissue block was cut into $10 \mu \mathrm{m}$ slices starting from the epicardium side. The histology slices were equivalent to the OCT en face imaging plane, i.e. the plane formed by the B- and C-scan directions at a specific depth from the epicardium. All tissue slices were numbered including damaged slices which, however, were not used in histology imaging. Tissue sections were stained with hematoxylin and eosin for microscopic imaging using a Nikon Eclipse E800 microscope equipped with a QImaging RETIGA 1300 camera.

\subsubsection{Fiber orientation measurement using $O P T$}

Our OPT method was implemented in a spectral domain full-range JMOCT system that has been described in detail previously (Fan \& Yao 2010b, 2012a). This bulk-optical system used a SLD source (SLD-351-HP, Superlum, Ireland) with a central wavelength of $847.8 \mathrm{~nm}$. A telecentric scan lens (LSM03-BB, Thorlabs, Newton, NJ) was used as the imaging objective. Jones matrix measurement was achieved by using two alternating incident polarization states: left- and right-circular polarization. An electro-optical modulator (EO-AM-NR-C1, Thorlabs, Newton, NJ) was used to modulate the incident polarization. For each incident polarization, the two orthogonal polarization components (vertical- and horizontal-polarization) of the backscattered signal were detected in the spectral domain using a custom spectrometer (Fan \& Yao 2010b, 2012a). A 1024-pixel 
line scan CCD camera (AVIIVA SM2, e2v, Milpitas, CA) was used as the detector.

The JMOCT system was carefully calibrated following a procedure described in (Fan \& Yao 2012a). The lateral resolution of the system was measured as $11.4 \mu \mathrm{m}$ and the depth resolution was measured as $8.1 \mu \mathrm{m}$ in air $(5.9 \mu \mathrm{m}$ in tissue with a refractive index of 1.38). The JMOCT system had an imaging speed of 50,000 A-lines/sec. One 3D scan covered an imaging volume of $1.1 \times 8.0 \times 8.0 \mathrm{~mm}^{3}$ with $280 \times 2000 \times 1000$ pixels in A-BC scan direction, leading to pixel sizes of $3.9 \mu \mathrm{m}, 4.0 \mu \mathrm{m}$, and $8.0 \mu \mathrm{m}$ in the $\mathrm{A}, \mathrm{B}$, and Cscan, respectively. In image processing, the 3D data set was resized using cubic spline to have the same pixel size of $3.9 \mu \mathrm{m}$ in all three dimensions. The measured system sensitivity was $108 \mathrm{~dB}$ and $104 \mathrm{~dB}$ at $0.5 \mathrm{~mm}$ and $1.0 \mathrm{~mm}$ from the zero delay line, respectively.

A Jones calculus based algorithm was used to derive the depth-resolved local retardance $(\delta)$, diattenuation $(\sigma)$, and optic axis orientation $(\theta)$ in addition to the intensity images (I). The retardance is defined as $2 \pi d \Delta \mathrm{n} / \lambda$ where $\Delta \mathrm{n}$ is the difference in refractive indices along the fast and slow optical axes and $d$ is the physical pathlength (pixel size). The diattenuation is defined as the difference in optical attenuation coefficients along the fast and slow optical axes. The "local" retardance and diattenuation were effective results averaged over a single image pixel. The detailed procedure to derive local polarization properties was described elsewhere (Fan \& Yao 2013). Briefly, each image pixel was modeled by a Jones matrix with an assigned set of polarization properties $(\delta, \sigma, \theta)$. The local retardance and diattenuation were first calculated from the measured cumulative Jones matrices at adjacent axial pixels using similar matrix transformation (Makita et al. 
2010; Fan \& Yao 2013). Then the local optic axis was calculated using an iterative algorithm starting from the sample surface (Fan \& Yao 2012b). The previously obtained local retardance was used to construct the local Jones matrix; whereas the local diattenuation was eliminated from the process (Fan \& Yao 2013). Fiber orientation was determined based on the angle of slow optic axis which ranged from $-90^{\circ}$ to $-90^{\circ}$ with the C-scan direction as the zero degree. To compare with histology results, the fiber orientation maps were constructed in the en face planes (B-C plane) at each depth with an axial pixel size of $3.9 \mu \mathrm{m} /$ pixel (Fan \& Yao 2013; Wang \& Yao 2013). To improve signal-to-noise, the obtained fiber orientation image in the $\mathrm{B}-\mathrm{C}$ plane was filtered using a $3 \times 3$ mean filter. The tractographic images were obtained by visualizing the optic axis images using the streamline or quiver functionalities in Matlab.

\subsubsection{Fiber orientation measurement using histology image}

Due to the labor intensive nature of histology analysis, an intensity-gradient based method (Karlon et al. 1998) was applied to calculate cardiac myofiber orientation in histology images to facilitate the comparison between OPT and histology images. This method has been previously applied to imaging fiber orientation in OCT intensity images (Fleming et al. 2008; Goergen et al. 2012; Gan \& Fleming 2013). The two-dimensional gradient at each image pixel was first computed using a $3 \times 3$ Sobel edge detector. Then the magnitude and direction of the gradient at each pixel was obtained as: 


$$
\begin{aligned}
& G(i, j)=\sqrt{G_{x}^{2}(i, j)+G_{y}^{2}(i, j)} \\
& \phi(i, j)=\tan ^{-1} \frac{G_{y}(i, j)}{G_{x}(i, j)}
\end{aligned}
$$

To improve signal-to-noise, the distribution of fiber orientation for all pixels within a sub-region ( $46 \times 46$ pixels) was assessed using the Von Mises distribution (Karlon et al. 1998). The most likely orientation corresponding to the peak of the Von Mises distribution was assigned to the center pixel within this region. The high resolution histology images had a pixel size of $0.25 \mu \mathrm{m} /$ pixel. A window of $46 \times 46$ pixels was equivalent to $11.5 \times$ $11.5 \mu \mathrm{m}^{2}$ which was the same as the OCT lateral resolution.

\subsubsection{Surface refraction correction}

The OPT and histology images were registered and compared as described below. First, the OPT image was transformed to correct the image distortion caused by surface refraction (Ortiz et al. 2010; Zhao et al. 2010). This is an important step to improve the accuracy of the direct comparison between histology and OPT images. As described previously in ophthalmology imaging (Ortiz et al. 2010; Zhao et al. 2010), optical refraction at tissue surface deviates the incident beam, which distorts the planar image plane. In addition, the

measured optic axis in JMOCT is within the imaging plane perpendicular to the light beam which may deviate from the original incident direction due to optical refraction at the same surface. As illustrated in Fig. 2-1, this plane is rotated away from the global en face B-C plane due to surface refraction and needs to be projected to the B-C plane (equivalent to 
the histology plane).

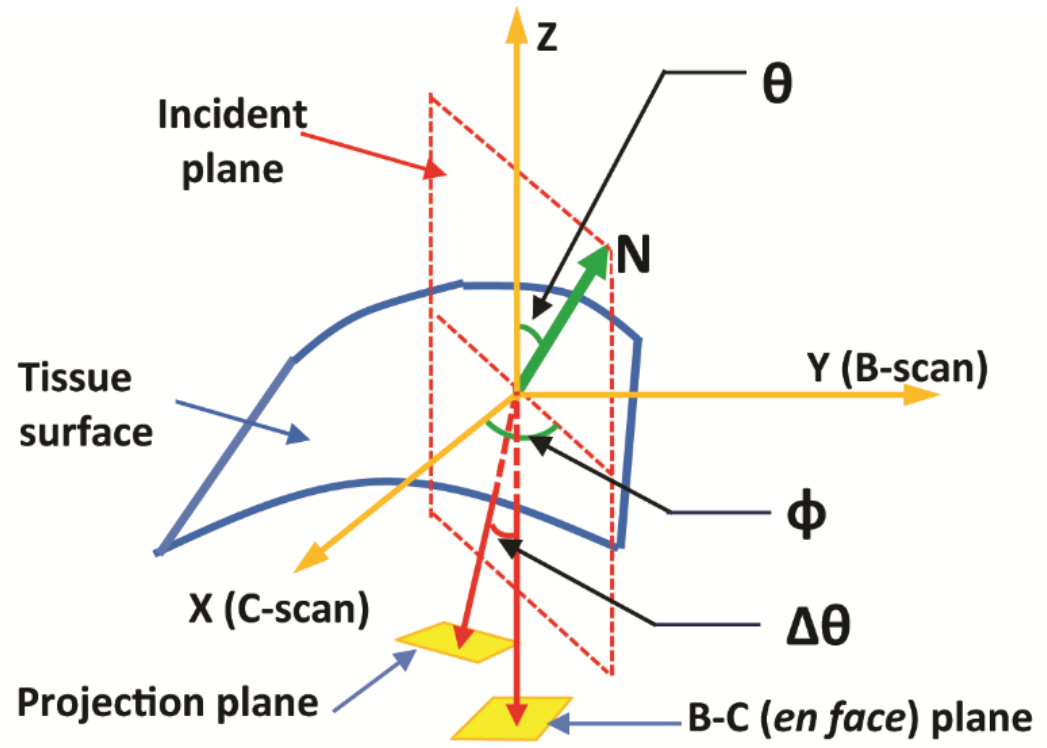

Figure 2-1. An illustration of image distortion induced by surface refraction. The incident light (A-scan) is aligned with the z-axis. $(\mathrm{N})$ is the surface normal vector at the light incidence point. The en face $\mathrm{x}-\mathrm{y}$ plane formed by the B- and C-scan are equivalent to the histology sectioning plane. The incident light is deviated by $\Delta \theta$ within the incident plane (formed by the incident light and the surface normal vector) due to optical refraction at the sample surface.

To correct surface refraction, the sample surface in the OCT intensity image was first determined using an intensity threshold-based segmentation algorithm (Fan \& Yao 2010b). The resulting surface data were represented as a 2D array containing the axial depth position for each pixel on the sample surface. We applied a $5 \times 5$ median filter to remove noisy pixels from the data set. The surface normal vector $\mathrm{N}=\left[\mathrm{N}_{\mathrm{x}}, \mathrm{N}_{\mathrm{y}}, \mathrm{N}_{\mathrm{z}}\right]$ was then calculated by using the "surfnorm" function in MATLAB. 


$$
\begin{aligned}
& \theta=\tan ^{-1} \frac{\sqrt{\mathrm{N}_{x}^{2}+\mathrm{N}_{y}^{2}}}{\mathrm{~N}_{z}} . \\
& \phi=\tan ^{-1} \frac{\mathrm{N}_{y}}{\mathrm{~N}_{x}}
\end{aligned}
$$

Due to surface refraction, the incident beam is deviated by and angle of $\Delta \theta$ within the incident plane which is formed by the incident light and the surface normal vector. Applying geometrical transformation, the actual pixel position ( $\left.\mathrm{x}^{\prime}, \mathrm{y}^{\prime}, \mathrm{z}^{\prime}\right)$ corresponding to the raw pixel $(\mathrm{x}, \mathrm{y}, \mathrm{z})$ can be calculated as:

$$
\begin{aligned}
& x^{\prime}=x+z \sin \Delta \theta \cos \phi \\
& y^{\prime}=y+z \sin \Delta \theta \sin \phi, \\
& z^{\prime}=z \cos \Delta \theta
\end{aligned}
$$

where the surface refraction induced direction change $\Delta \theta$ can be determined according to the Snell's law at the tissue surface:

$$
\Delta \theta=\theta-\sin ^{-1}\left(\frac{\sin \theta}{n}\right)
$$

A value of 1.38 was used as the tissue refractive index $n$ in our calculation. The fiber orientation angle $\beta^{\prime}$ measured in the global en face B-C plane can then be calculated as:

$$
\beta^{\prime}=\tan ^{-1}\left(\frac{\cos \Delta \theta \cos \varphi \sin \beta-\sin \varphi \cos \beta}{\cos \Delta \theta \sin \varphi \sin \beta+\cos \varphi \cos \beta}\right),
$$

where $\beta$ is the directly measured orientation angle. The above correction procedure was 
applied to all pixels in the 3D OPT dataset.

\subsubsection{Image registration}

The orientation of each histology image had random variations during the process of sectioning and mounting of histology slides. Therefore all histology images need to be aligned with each other. We first registered all histology images based on their boundary profiles obtained using a low magnification $2 \times$ objective lens. A Matlab program was developed using the function "imregister" to register all histology images. This intensitybased automatic registration uses an optimization algorithm to find the best transformation to register two input images. We used "rigid" transformation (translation and rotation) to register all $2 \times$ histology images. The pixel-wise mean square error was used as the

optimization metric and an optimizer with a gradient magnitude tolerance of $10^{-4}$ and 300 iterations was used as the converging condition.

In addition, all histology images were registered with OCT intensity images using the same algorithm. Before applying the registration, the histology images were rescaled to have the same pixel resolution as the OCT images. The obtained translation and rotation values $\left(t_{B}, t_{C}, \alpha\right)$ for each histology slice were stored, where $t_{B}$ and $t_{C}$ are translational distances (in pixel) along the B- and C-scan, respectively, and $\alpha$ is the rotation angle along the A-scan direction. In order to obtain accurate fiber orientation from histology images, a $40 \times$ objective lens was used to image the histology samples so that individual fibers can be resolved. After the fiber orientation was calculated using the algorithm described in Sec. 
2.2.3, the stored transformation $\left(\mathrm{t}_{\mathrm{B}}, \mathrm{t}_{\mathrm{C}}, \alpha\right)$ was applied to register the histology tractographic image with OPT image.

\subsection{Results}

Figure 2-2 shows an example of the heart tissue image obtained in this study using the JMOCT system.
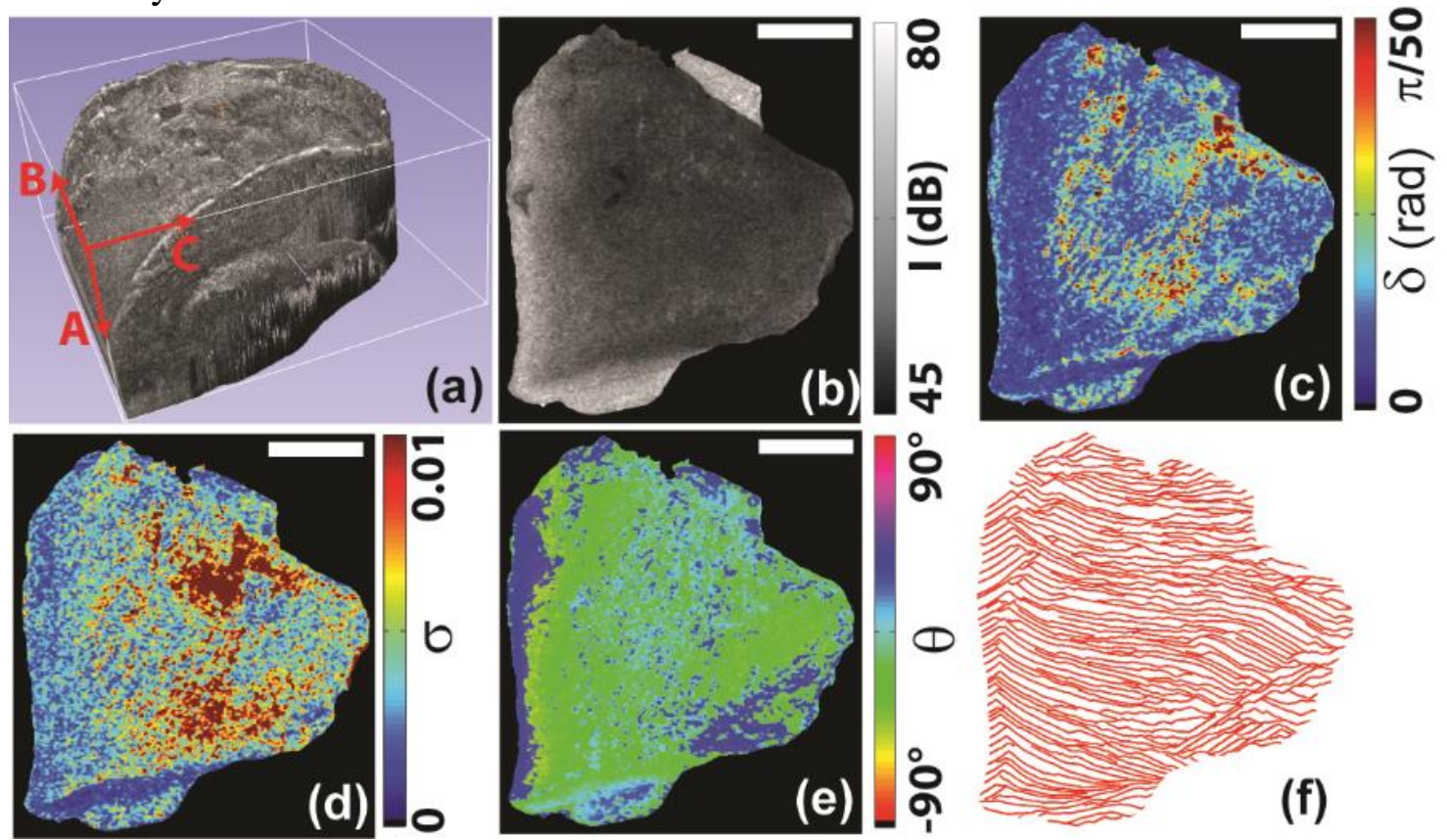

Figure 2-2. A 3D image of a piece of heart tissue. (a) The 3D OCT intensity images. The example en face images of (b) intensity (I), (c) local retardation $(\delta)$, (d) local diattenuation $(\sigma)$, and (e) local optic axis $(\theta)$ extracted from a depth of $0.56 \mathrm{~mm}$ from surface. (f) A tractographic representation of fiber orientation using the streamline plot. The size bars in the images represent $0.5 \mathrm{~mm}$.

The 3D OCT image of this piece of heart tissue is shown in Fig. 2-2(a) with the A, B-, and C-scan directions marked. Figures 2-2(b-f) show examples of en-face images (within the B-C plane) extracted at $\sim 0.56 \mathrm{~mm}$ beneath the epicardium surface. The 
measured average local retardation (Fig. 2-2(c)) was $1.72 \times 10^{-2} \pm 9.8 \times 10^{-3} \mathrm{rad} / \mathrm{pixel}$. The measured average diattenuation (Fig. 2-2(d)) was $5.5 \times 10^{-3} \pm 4.7 \times 10^{-3} /$ pixel. As explained in Sec. 2.2.2, these "local" values represented the effective tissue polarization properties within the corresponding single image pixel and were similar to those reported previously (Wang \& Yao 2013). No fibrous structure can be discerned in the OCT intensity image (Fig. 2-2(b)) due to insufficient spatial resolution to resolve the individual muscle fibers in the mouse heart. However, the optic axis can still be clearly measured as shown in the pseudocolor image in Fig. 2-2(e). The streamline plot of the optic axis data was used to construct the tractographic representation of the fiber orientation (Fig. 2-2(f)).

\subsubsection{Correcting surface refraction}

The image distortion due to surface refraction can significantly impact the OPT accuracy. To illustrate this effect, Fig. 2-3(a) shows an example histology image obtained at a depth of $0.35 \mathrm{~mm}$ beneath the epicardium. Figure 2-3(b) shows the fiber orientation image obtained from the histology image as described in Sec. 2.2.3. Figures 2-3(c) and 2-3(d) show the OPT results before and after correcting the surface refraction.

Without correcting the surface refraction, the OPT results (Fig. 2-3(c)) were significantly different from the histology tractography (Fig. 2-3(b)). For example, the central part of the OPT shows positive orientations; whereas the orientation revealed in histology is close to zero degree. In addition, the fibers in the upper left corner of the histology result (Fig. 2-3(b)) show positive angles; whereas they remain almost horizontal in OPT (Fig. 2-3(c)). After applying the correction as describe in Sec. 2.2.4, the resulting 
OPT (Fig. 2-3(d)) appears very similar to the histology results (Fig. 2-3(b)). We noticed that most of the improvement was due to the position correction using Eq. (3). The orientation change (Eq. (5)) only induced minimal changes in the resulting orientation, likely due to the relative flat surface of the tissue samples.
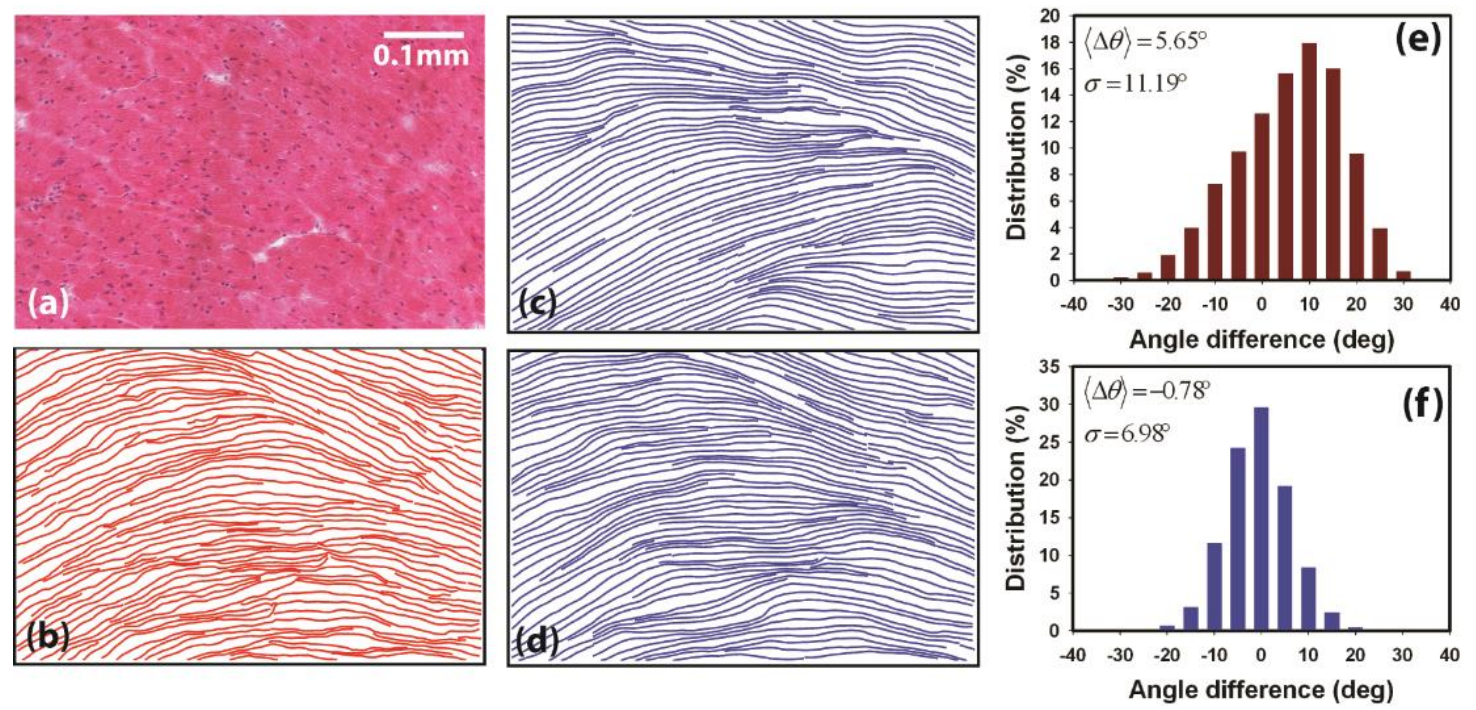

Figure 2-3. An example of the surface refraction correction. (a) The histology image obtained at $0.35 \mathrm{~mm}$ beneath the epicardium surface. (b) The tractograph obtained from the histology image. (c) The OPT result without correcting the surface refraction. (d) The OPT after surface refraction correction. The corresponding distribution of pixel-by-pixel difference between histology and OPT before and after correction are shown in (e) and (f), respectively.

To further quantify the differences between histology and OPT, the pixel-by-pixel differences between the two images were calculated. The histology images were resized using cubic spline to match the pixel size of $3.9 \mu \mathrm{m}$ in OPT. As shown in Fig. 2-3(e) and 2-3(f), the mean difference between histology and OPT was reduced from $5.65^{\circ}$ before correction to $-0.78^{\circ}$ after correction. The correction reduced standard deviation from 
$11.19^{\circ}$ to $6.98^{\circ}$. After the distortion correction, about $85.5 \%$ of the 14,959 pixels in this image area (Fig. 2-3(a)) had $\mathrm{a} \leq 10^{\circ}$ difference in measured orientation between OPT and histology. Even though this pixel-wise comparison did not reach a perfect $100 \%$ match, the corrected OPT (Fig. 2-3(d)) and histology result (Fig. 2-3(b)) resembled each other remarkably well. As explained later in this section, tissue variations introduced during histology processing made it extremely challenging to obtain a perfect pixel-wise match.

\subsubsection{Depth profile of fiber orientation}

A major feature of the myofiber architecture in heart is the unique "cross-helical" pattern where the myocardial fibers rotate from negative angles at epicardium to positive angles at endocardium (LeGrice et al. 1995a). To verify OPT, we calculated the depth profile of fiber orientation in each animal tissue and compared the results with histology. A total of 21 small regions of interest (ROIs) with a size of $92 \times 92 \mu \mathrm{m}^{2}$ each were evaluated with 3 ROIs selected from each mouse heart. The average value and standard deviation of fiber orientation were obtained using both histology and OPT images from the epicardium to 1$\mathrm{mm}$ in depth. Because the high resolution histology images of these ROIs were acquired using a $40 \times$ objective, they had a very limited field of view. To ensure a consistent ROI position in each histology image at different depths, their positions were carefully examined in relation to the tissue boundaries among adjacent histology slices and with the assistance of image registration as described in Sec. 2.2.5. As explained in Sec. 2.2.1, the histology slices had a thickness of $10 \mu \mathrm{m}$, whereas OPT had an axial pixel size of $3.9 \mu \mathrm{m}$. To facilitate the comparison, cubic spline interpolation was applied to resize the OPT axial 
pixel to the same size of $10 \mu \mathrm{m}$ as in histology.
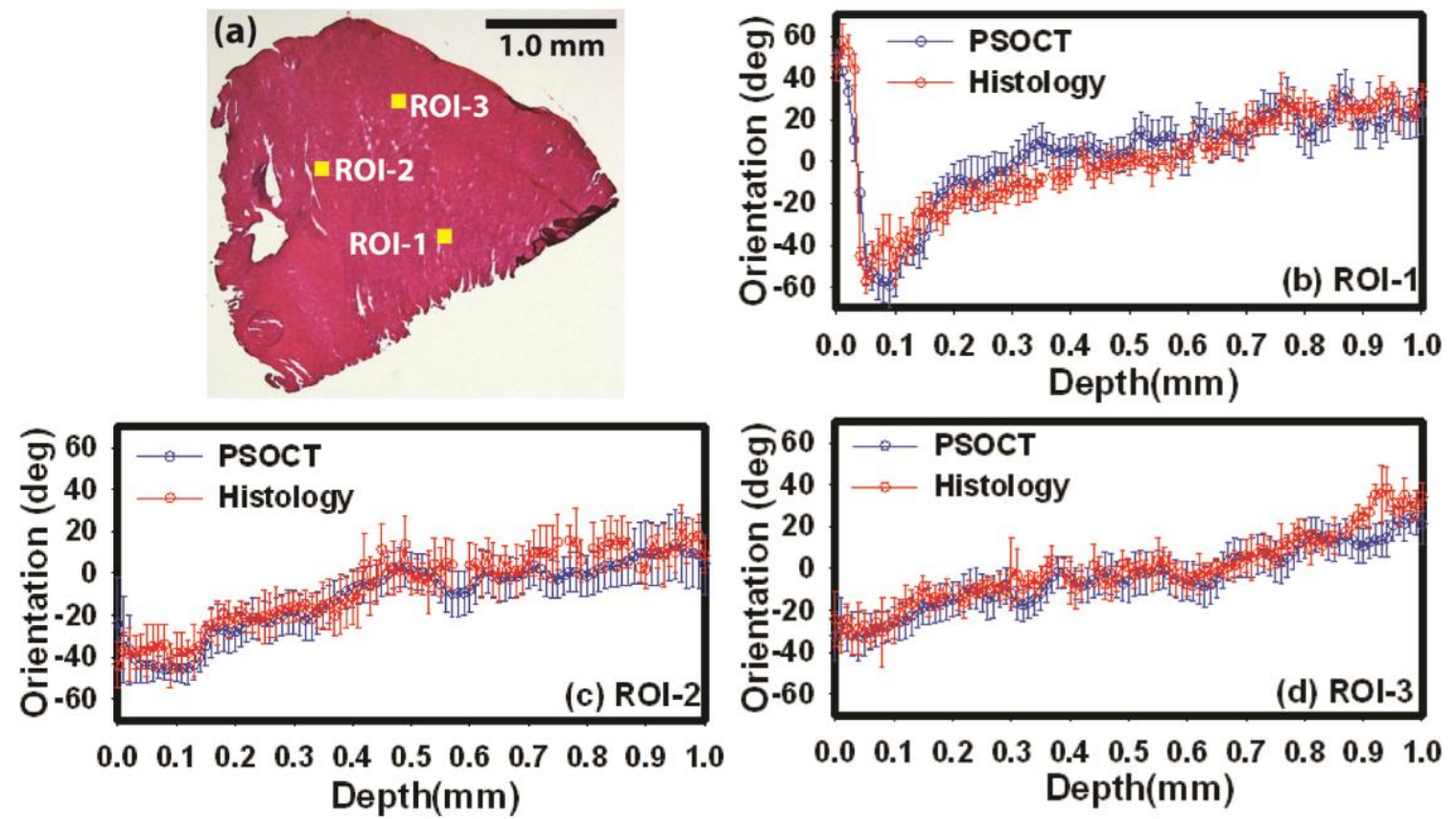

Figure 2-4. A direct comparison of the depth-resolved myocardiac fiber orientation measured from histology and OPT. The three ROIs were selected from the heart sample as shown in (a). The size of the ROI is $92 \times 92 \mu \mathrm{m}^{2}$. Error bars indicate standard deviation.

Figure 2-4 shows an example depth profiles of fiber orientation obtained from 3 ROIs in a piece of heart sample. This tissue sample was excised from the left ventricle of the mouse heart. There were a few data points missing in the histology results due to damaged tissue slices during sectioning. The fiber orientation in ROI-2 and 3 had similar depth profiles with fibers oriented at $-30^{\circ}$ to $-50^{\circ}$ at epicardium and transiting to $+20^{\circ}$ to $+40^{\circ}$ at $1.0 \mathrm{~mm}$ depth following an approximately linear trend. At the location of ROI-1, however, the orientation of myocardial fibers showed a different depth profile. The fibers 
had a positive orientation at epicardium and rotated clockwise to negative angles at $\sim 50$ $\mu \mathrm{m}$. The fibers then reversed the trend and started to rotate counterclockwise and eventually reached to $\sim-20^{\circ}$ at $1 \mathrm{~mm}$ beneath the epicardium. Despite the differences, all three curves revealed the typical global cross-helical structure in cardiac muscle, i.e. a transition from negative fiber orientation close to epicardium to positive fiber orientation toward endocardium. These curves transitioned from negative angles to positive angles at $\sim 0.5$ $\mathrm{mm}$.

Overall, the OPT and histology results agreed very well in these three ROIs. In addition, the standard deviation obtained in each ROI was comparable in both histology and OPT results. Quantitatively the OPT and histology results were highly correlated with each other. The obtained coefficients of determination were $\mathrm{R}^{2}=0.88,0.94,0.90$ for ROI1, 2, and 3, respectively. As discussed in our previous study (Wang \& Yao 2013), the reversed trend in ROI-1 observed within the first $50 \mu \mathrm{m}$ beneath the epicardium was not previously reported in the diffusion tensor MRI (DTI) images of mouse heart, most likely due to the insufficient spatial resolution in DTI. Nevertheless this observation has been clearly validated in histology images (Fig. 2-4(b)).

Figure 2-5(a) shows the overall correlation $\left(\mathrm{R}^{2}=0.85\right)$ of the OPT and histology results obtained in all seven mice. The entire data set had over 2,000 data points obtained from a total of 21 depth profiles from 0 to $1.0 \mathrm{~mm}$ depth with a step size of $10 \mu \mathrm{m}$. Figure 2-5(b) shows the distribution of the difference in orientation angles calculated using these two methods. The obtained histogram can be well fitted using a Gaussian function (adj- 
$\left.\mathrm{R}^{2}=0.994\right), a \times \exp \left[-\left(x-x_{0}\right)^{2} /\left(2 \sigma^{2}\right)\right]$. The fitted mean difference is $x_{0}=1.0^{\circ}$ with a standard deviation of $\sigma=9.1^{\circ}$. A total $82.2 \%$ of the data points had a difference $\leq 10^{\circ}$. Such a level of consistence between OPT and histology exceeded the correlation between histology and diffuse tensor based MRI reported in a previous study (Hsu et al. 1998).
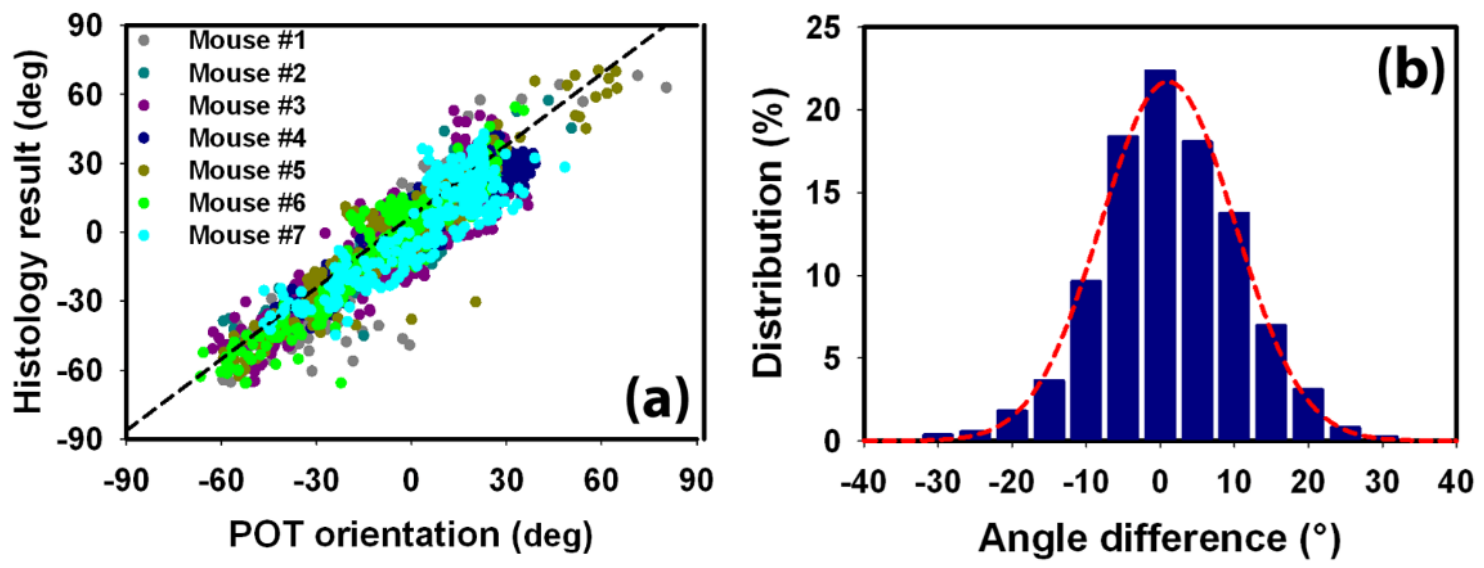

Figure 2-5. The correlation results between OPT and histology. (a) The linear correlation between OPT and histology results $\left(\mathrm{R}^{2}=0.85\right)$. (b) The distribution of the difference between the fiber orientation angles measured using OPT and histology. The data was depth-resolved orientation (Fig. 2-4) measured from a total of 21 ROIs selected from the heart samples in 7 mice. The red dashed line in (b) represents a Gaussian fitting.

\subsubsection{Pixel-wise comparison between OPT and histology}

In order to better understand the differences between OPT and histology measurements, we investigated the pixel-wise differences between the two tractographic images. The high resolution histology images acquired using a $40 \times$ objective had a limited field of view of $256 \times 320 \mu \mathrm{m}^{2}$. To obtain the histology image of a large histology slice, multiple overlapping histology images of the same slice were acquired and then manually stitched together using the Photoshop software. Because this manual stitching process is time 
consuming, we constructed the large histology images at two depths of $\sim 0.35 \mathrm{~mm}$ and $\sim 0.65 \mathrm{~mm}$ in each tissue sample. To register these large histology images with OPT images, the image registration described in Sec. 2.2.5 was fine-tuned by transforming the histology images within a $50 \mu \mathrm{m}$ translational range and $5^{\circ}$ rotational range around the original transformation parameters $\left(\mathrm{t}_{\mathrm{B}}, \mathrm{t}_{\mathrm{C}}, \alpha\right)$ obtained using sample boundary profiles. The final registration transformation was selected if it produced the minimal root-mean-square (RMS) value of the pixel-by-pixel difference between the histology and OPT. The resulting adjustments in the 14 images ( 2 evaluation depths in each of the 7 samples) were between $-27.3 \mu \mathrm{m}$ to $+35.1 \mu \mathrm{m}$ in translation and $-2.2^{\circ}$ to $+1.3^{\circ}$ in rotation. These small adjustment values suggested the good accuracy in the original image registration.

Figure 2-6 shows an example comparison between the histology tractography and OPT obtained in a tissue sample excised from the right ventricle of a mouse heart. The tractography was overlaid over corresponding histology and OCT intensity images. The size of the image is $1.0 \times 1.0 \mathrm{~mm}^{2}$. The histology images were resized to have the same pixel size of $3.9 \mu \mathrm{m}$ as the OPT images. The muscle fibers at $0.34 \mathrm{~mm}$ beneath the epicardium showed a trapezoid type organization, i.e. the fibers entered at the left side with positive angles, transited into horizontal orientation at the middle, and then left the area with negative angles from the right side. In comparison, the fiber organization at $0.66 \mathrm{~mm}$ beneath the epicardium appeared more homogeneous with all fibers crossing the entire area with positive orientations. 

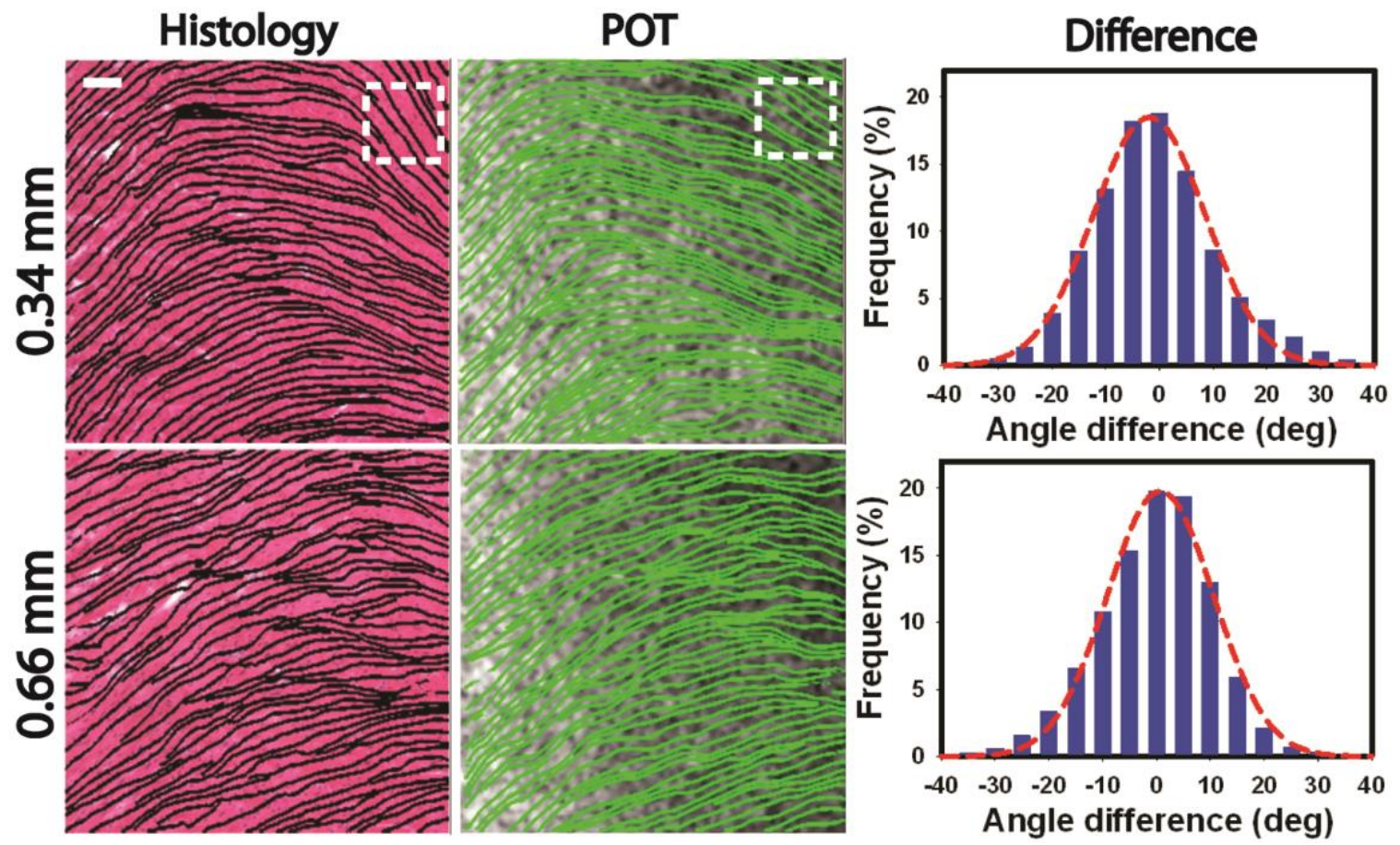

Figure 2-6. An example comparison of tractography computed using OPT and histology at depths of $0.35 \mathrm{~mm}$ and $0.65 \mathrm{~mm}$ from the epicardium in a heart tissue sample. The tractography was overlaid over histology and OCT intensity images. Also shown are the corresponding distributions of the pixel-by-pixel orientation difference, in which the dashed lines were fitting results using a Gaussian function. The white dashed boxes highlight the difference between histology and OPT. The size bar indicates $100 \mu \mathrm{m}$.

Overall, very similar fiber orientation patterns were revealed in both histology and OPT results. The distributions of the pixel-by-pixel difference were calculated for a quantitative comparison. As shown in Fig. 2-6, both distributions fitted well $\left(\mathrm{R}^{2}>0.99\right)$ using a Gaussian function. The fitting mean difference and standard deviation are $\mathrm{x} 0=-$ $1.9^{\circ}, \mathrm{b}=10.4^{\circ}$ for $0.34 \mathrm{~mm}$ depth and $\mathrm{x} 0=-0.7^{\circ}, \mathrm{b}=9.9^{\circ}$ for $0.66 \mathrm{~mm}$ depth. At the 0.34 $\mathrm{mm}$ depth, $63.5 \%$ of the 65,536 pixels had $\leq 10^{\circ}$ difference between OPT and histology; whereas the percentage was slightly higher at $67.9 \%$ for the results obtained at the depth 
of $0.66 \mathrm{~mm}$. If the difference threshold was set as $15^{\circ}$, the percentage became $80.9 \%$ and $85.7 \%$ for $0.34 \mathrm{~mm}$ and $0.66 \mathrm{~mm}$, respectively. Among all 14 large scale comparisons, the percentage of pixels with $\leq 15^{\circ}$ difference between OPT and histology ranged from $69.9 \%$ to $95.5 \%$.

\subsubsection{Error analysis}

Each of these large images was visually examined in detail to understand the difference between histology and OPT at pixel level. For the sample shown in Fig. 2-6, despite the overall good agreement between OPT and histology, there is a difference at the upper right corner (marked with dashed lines) of the tractography obtained at $0.34 \mathrm{~mm}$. Specifically, the fibers in OPT within the box in Fig. 2-6 were more inclined toward the horizontal direction. A close analysis revealed that this difference was due to imperfect alignment between the en face OPT and histology slices. Although the histology processing was prepared by an experienced staff and under great care, the histology-sectioning plane may be slightly different from the en face plane (Fig. 2-7(a)). Indeed, as shown in Figs. 2-7(ad), the difference in the aforementioned upper-right corner in Fig. 2-6 was significantly reduced after moving the slice position $35 \mu \mathrm{m}$ toward the epicardium (tilting the en face plane for $\sim 2^{\circ}$ along the diagonal line in Fig. 2-6).

In addition, we found that many of the differences between OPT and histology tractography can be attributed to those incorrect fiber orientations obtained in histology analysis. The edge detection used to identify fiber structures was also affected by any intensity changes that are not related to muscle fibers. For example, vessels, scars or tissue 
damage from histology processing all appeared with different pixel intensities in histology images. As an example, Fig. 2-7(e) shows a tissue section with a cluster of nuclei and lightcolored tissue components at the center (circled with a dashed line). To facilitate the comparison, both tractographic results were visualized using quiver plot and overlaid on the histology image. Evidently, these fiber irrelevant spatial intensity variations produced incorrect fiber orientation data in the high resolution histology tractography. On the other hand, optic axis measurement is independent of such intensity variations in the sample. As a result, the OPT result appeared to follow the fiber tracts correctly (Fig. 2-7(f)). Unfortunately such incorrect histology fiber tracking contributed to the difference between histology tractography and OPT.

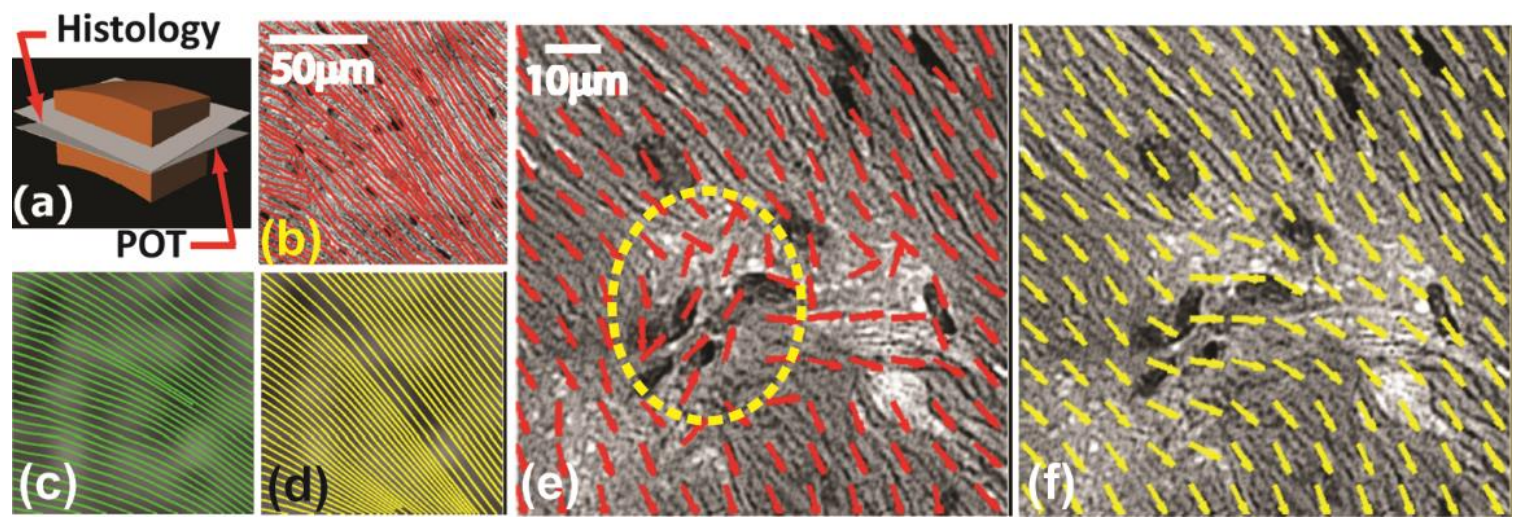

Figure 2-7. Analysis of possible sources of error. (a) The potential misalignment between the histology sectioning plane and en face OCT scanning plane. (b) The histology tractography and (c) OPT in a tissue sample located at $0.35 \mathrm{~mm}$ beneath the epicardium. (d) OPT results of the same tissue region with the en face plane tilted $2.0^{\circ}$. (e) The high resolution histology tractography and (f) OPT in a tissue region with intensity variations.

Finally, it is known that the geometric shape of the tissue and its fiber organization may be altered during the histology processing. The histology sectioning process may 
cause tissue deformation and even tearing. At the proxy of these alterations, the tissue morphology was changed. Although these changes may not significantly impact the overall fiber organization, they greatly affected the pixel-wise comparison. We observed that large pixel-by-pixel differences between OPT and histology may appear at location with significant changes in fiber organization, e.g. fiber transition, splitting and merging. At such locations, any small morphological alteration may induce a large pixel-wise difference due to the high spatial resolution. Figure 2-8 shows an example comparison of a $1.0 \times 1.0 \mathrm{~mm}^{2}$ tissue from the left ventricle of a mouse heart. This pair of images had the largest overall pixel-by-pixel difference among all samples we examined. Only $69.9 \%$ of the pixels in the image had a difference $\leq 15^{\circ}$ between histology and OPT. Still, the OPT reliably reproduced all key features of fiber organization in the piece of sample as shown in Fig. 2-8.

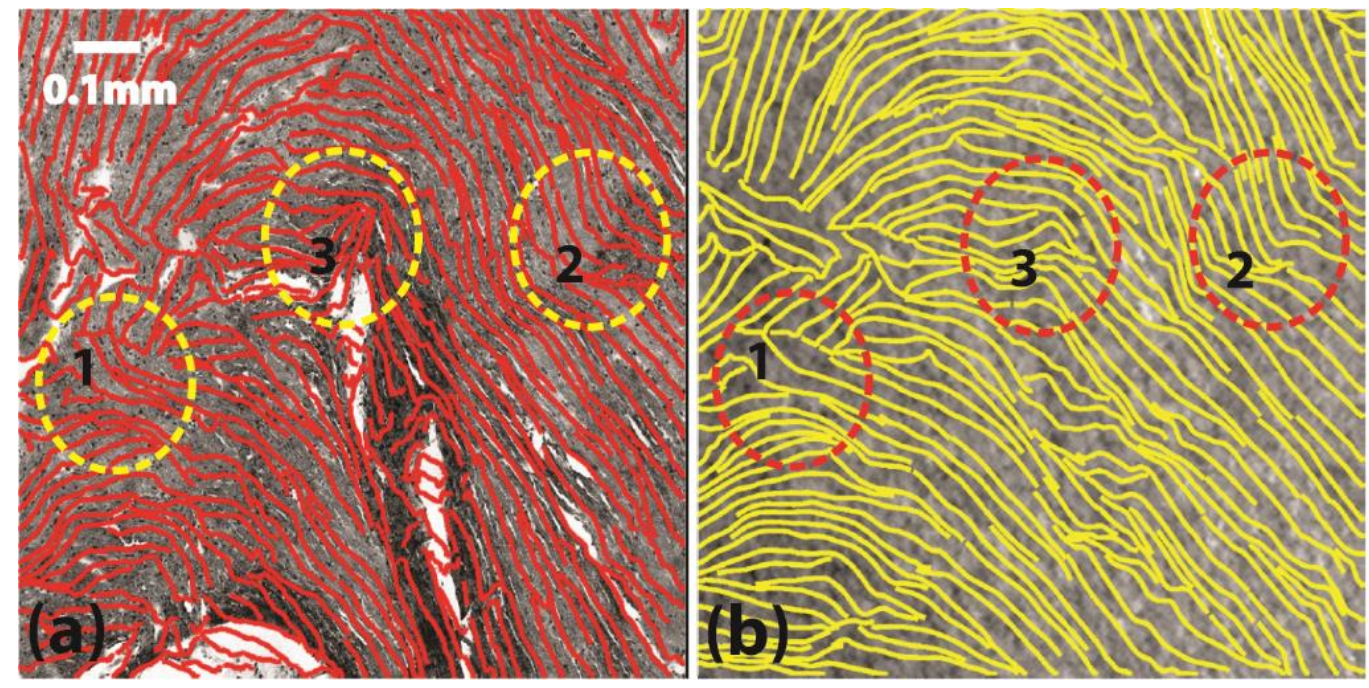

Figure 2-8. A comparison of the (a) histology tractography and (b) OPT in a tissue sample with significant amount of orientation changes and tissue deformation caused by histology processing. 
The fiber transitions marked by dashed circles (\#1 and \#2) at the left and right sides of the images were very similar in both images. Tissue tearing appeared in the middle part of the histology image (Fig. 2-8(a)), which was coincident with the location showing the largest differences between the two tractography results. Such tissue tearing induced morphological changes in the surrounding region. As a consequence, the fiber organization in the middle region (circle \#3) appeared similar but the exact shape and position were changed. Such image deformation at microscopic scales may be more accurately handled using more sophisticated image registration algorithms (Rohde et al. 2003). Nevertheless, OPT and histology results remained consistent in regions not affected by such histology artifacts.

\subsection{Conclusions}

In this study, we compared depth-resolved fiber orientation mapping in fresh heart samples obtained by using OPT and histology images. We developed a systematic set of image processing methods to correct image distortions in OPT induced by surface optical refraction and to register OPT with histology images. The results indicated that OPT can reliably map the cardiac myofiber organization in fresh mouse heart. The fiber orientation measured in OPT agreed very well with histology results. A detailed pixel-wise analysis indicated that the majority of the differences between OPT and histology can be attributed to imperfect alignment between histology and OPT, tissue alterations during histology 
processing, as well as artifacts when extracting fiber orientation in histology images. In addition, deteriorating signal to noise at larger depths eventually led to inaccurate fiber orientation calculation. As a result, the image depth of our current system was limited to $1.0 \mathrm{~mm}$ in fresh heart tissue. Nevertheless, the OPT technology opens a new way for imaging the important cardiac myofiber architecture in fresh heart samples. With its combined advantages of fast speed, large field of view and high resolution, OPT is especially attractive for imaging applications in small animal models. If implemented in a fiber optics based PSOCT system, this technology may be integrated into catheter based system for in vivo endoscopic imaging. 


\section{Chapter 3. Tractographic imaging of the mouse heart using OPT ${ }^{\dagger}$}

\subsection{Introduction}

Histological studies (Streeter \& Bassett 1966; Streeter Jr et al. 1969) have revealed the "cross-helical" structure of myocardial fibers formed laminarly at different transmural thicknesses in the ventricular myocardium of the heart (LeGrice et al. 1995a). This unique fiber architecture has a great influence on mechanical and electrical heart functions (Taccardi et al. 1994; LeGrice et al. 1995b). Abnormal fiber organization is linked to cardiac dysfunction and alterations in cardiac muscle structure have been found as a result of myocardial infarction (Strijkers et al. 2009). Therefore "tractography", an imaging tool that can acquire microstructural details of tissue fiber organization, is valuable for both basic research and clinical diagnosis. Despite its superior spatial resolution, histological assessment is time consuming and limited to imaging of small areas. Alternatively, diffusion-tensor magnetic resonance imaging (DTI) (Sosnovik et al. 2009a) has been established as a state-of-art method for imaging the 3D fiber organization in whole hearts; however, the image resolution in DTI is usually limited to submillimeters.

Optical coherence tomography (OCT) is a non-destructive optical imaging

\footnotetext{
$\dagger$ This chapter was from a published article: Wang, Y. and Yao, G., 2013. Optical tractography of the mouse heart using polarization-sensitive optical coherence tomography. Biomedical optics express, 4(11), pp.2540-2545.
} 
technique that can provide depth-resolved high resolution tissue images at high speed. It has been shown that ultrahigh resolution OCT can be used to acquire fiber orientation in heart tissue using intensity contrast (Fleming et al. 2008; Ambrosi et al. 2012; Goergen et al. 2012). Because fibrous tissues such as myocardium show intrinsic optical birefringence, fiber orientation can be estimated using the optic axis' information. Recently, a set of algorithms (Fan \& Yao 2012c, b, 2013) have been developed to extract the depth-resolved local optical polarization properties from polarization-sensitive optical coherence tomography (PSOCT) images. Fan and Yao (Fan \& Yao 2013) demonstrated that these algorithms can be applied in a Jones matrix PSOCT system (Fan \& Yao 2012a) to visualize myocardial fiber orientation in a piece of bovine heart tissue.

Here, we further extend our previous studies by developing a new scanning and image reconstruction procedure to acquire tractography in whole mouse hearts. The wellknown "cross-helical" laminar structure of myocardial fibers in the ventricular myocardium can be clearly visualized using this technology and the obtained myocardial fiber organization agreed well with existing knowledge acquired using diffusion tensor magnetic resonance imaging.

\subsection{Method}

The Jones matrix PSOCT system used in this study is a single camera-based full-range spectral domain system as described in detail in (Fan \& Yao 2013). This system was 
carefully calibrated for imaging conventional 'cumulative' polarization properties including retardance (or 'phase retardation'), optic axis and diattenuation (Fan \& Yao 2013). A Superluminescent Light Emitting Diode (SLD) was used as the light source (SLD-351-HP, Superlum, Ireland) at a central wavelength of $847.8 \mathrm{~nm}$. The system had a depth resolution of $8.2 \mu \mathrm{m}$ in the air within $1.5 \mathrm{~mm}$ of the zero delay line as characterized using the interference signal from a mirror at the sample arm. The lateral resolution of the system was $12.4 \mu \mathrm{m}$ when measured using a 1951 USAF test target. All size/distance values presented in this report were measured in air.

The excised mouse heart was fixed in $4 \%$ paraformaldehyde and imaged ex vivo. A 20 gauge needle was used to hold the heart by passing through the heart apex and center of the base. The needle was mounted on a rotational stage (PRM1Z8, Thorlabs, Inc., Newton, New Jersey, USA) and was aligned with the rotational axis. The stage was rotated continuously over $270^{\circ}$ at a speed of $1.25^{\circ} / \mathrm{sec}$. This rotation range was chosen to ensure that the entire left ventricle and part of the right ventricle can be imaged within the effective image depth of $2.6 \mathrm{~mm}$ of the PSOCT system (Fan \& Yao 2012a). A total of 2700 B-scans (2000 pixels in each B-scan covering $7.0 \mathrm{~mm}$ ) were acquired at a speed of $12.5 \mathrm{~B}$-scans per second ( $18 \mu$ s camera exposure) to match the rotation speed. The entire scanning took 216 seconds to complete.

A coordinate system was set up to illustrate the measurement geometry as shown in Fig. 3-1. The incident light was aligned with the Z-axis (A-scan) and the B-scan was aligned with the $\mathrm{Y}$-axis (parallel to the axis of rotation). The XY plane, i.e. the imaging 
plane for axis measurement, was perpendicular to the Z-axis and was set up as an analog to a histology slice. The projection angle of myocardial fibers passing through a pixel within the XY plane was measured by extracting the local (slow) optic axis in relation to the $\mathrm{x}$-axis from the PSOCT images. The measured orientation $\theta$ had a range of $\left[-90^{\circ}, 90^{\circ}\right]$ with positive angles inclined toward the positive x-axis (Fig. 3-1).

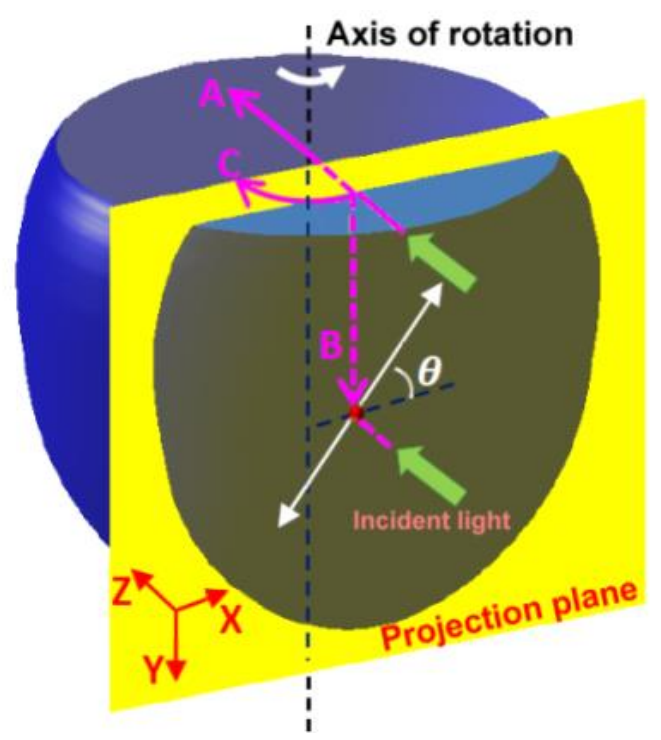

Figure 3-1. An illustration of the imaging geometry. The incident light (A-scan) was aligned with the z-axis; the B-scan was along the axis of rotation (y-axis); and the heart sample was rotated for C-scan.

The procedure for extracting local optic axis, retardance and diattenuation from PSOCT was detailed in (Fan \& Yao 2013). The term "optic axis" used here refers to the "slow" optic axis which is aligned with the fiber orientation. Briefly, the two orthogonal (horizontally and vertically polarized) components of the backscattered OCT signal were measured for both right- and left-handed circularly polarized incident light. The measured 
signals were stored in a "planar" 3D matrix of $650 \times 2000 \times 2700$ pixels with pixel sizes of $5.4 \mu \mathrm{m} \times 4 \mu \mathrm{m} \times 7.9 \mu \mathrm{m}$ in A-, B-, and C-scans, respectively. A birefringent Jones matrix was constructed at each image pixel in the dataset with tissue diattenuation removed by using only the real component of the measured complex retardance (Fan \& Yao 2013). To improve signal-to-noise, the amplitudes of the two orthogonal polarization components and their phase difference were averaged over 3 consecutive B-scans during the calculation. In addition, a size three median filter $(3 \times 3 \times 3)$ was applied to the $3 \mathrm{D}$ dataset to further reduce speckle noise. Then, an iterative algorithm (Fan \& Yao 2012b, 2013) was used to calculate the local depth-resolved optic axis.
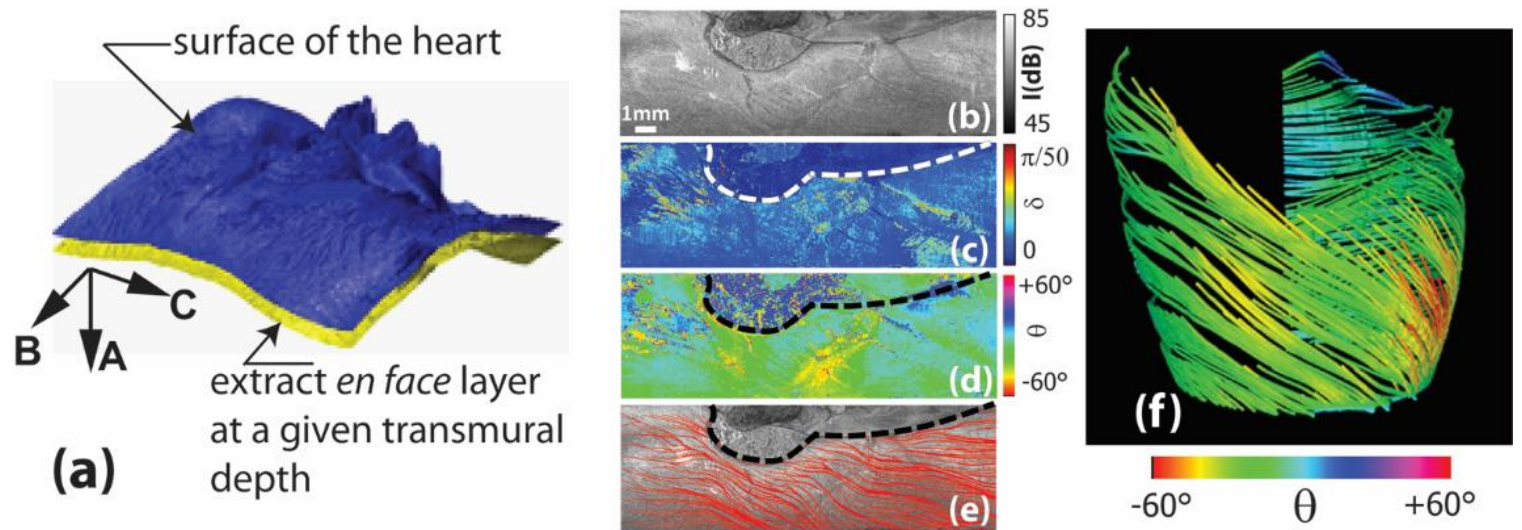

Figure 3-2. An illustration of the procedure for reconstructing the tractography in a mouse heart. (a) Extracting an en face image at a given transmural depth. The planar presentation of the extracted en face images of (b) intensity, (c) local retardance, and (d) local fiber orientation; (e) the streamline presentation of fiber tract overlaying on the intensity image; (f) the reconstructed $3 \mathrm{D}$ tractographic representation of the fiber tracts in (e).

Figure 3-2 illustrates the procedure for constructing the 3D fiber tract from the local optic axis dataset. The sample surface boundary was first obtained from the intensity data for each A-scan using a threshold-based segmentation algorithm (Fan \& Yao 2012a). Then, 
depth-resolved en face images were constructed from all pixels at a given transmural depth from the surface boundary (Fig. 3-2(a)). Examples of the extracted "planar" en face PSOCT images (intensity, local retardance, and optic axis) are shown in Fig. 3-2(b-d). As shown in Fig. 3-2(c), the artery tissues (enclosed by the dashed line) had low retardance values which led to unreliable optic axis calculation. To focus on quantifying myocardial fibers in ventricles, the artery and atrium tissues were removed from further processing using a retardance threshold of 0.012 rad at the boundary of the ventricle as shown in Fig. 3-2(ce).

Within each en face image of the local optic axis (Fig. 3-2(d)), the stream2 function in Matlab was used to obtain the "streamline" representation of fiber tracts (Fig. 3-2(e)) (Fan \& Yao 2013). A uniform $25 \times 34$ mesh grid was used as the starting points in streamline calculation of the whole mouse heart. To improve the tractographic visualization, a cubicspline interpolation algorithm was applied to each obtained fiber tract to fill the empty space between the tracking seeds so that each fiber tract contained 2700 points. The obtained fiber tract was further smoothed using an averaging filter with $1 \%$ of the given fiber length as the window size. To help eliminate incorrect fiber tracts produced by noisy data, short fiber tracts whose lengths were less than $5 \%$ of C-scan length were removed from visualization.

Once the "planar" orientation map (Fig. 3-2(e)) was obtained, it was then transformed into 3D coordinates using a polar transformation. The polar angle $\alpha_{\mathrm{i}}$ at $\mathrm{C}$-scan index $i$ was computed as: $\alpha_{i}=\left(i / N_{C}\right) \times \Phi$, where $\mathrm{N}_{\mathrm{C}}$ denotes the total number of C-scan 
and $\Phi$ is the full rotation range angle $\left(270^{\circ}\right.$ in this study). The radius $r$ in the polar transformation was computed as $r=R_{0}-\operatorname{Surf}\left(C_{i}, B_{j} A_{k}\right)$, where $\mathrm{R}_{0}$ denotes the maximum radius of the sample heart within the $\mathrm{XZ}$ plane (Fig. 3-1) and was $4.5 \mathrm{~mm}$ for the same presented here. $\operatorname{Surf}\left(C_{i}, B_{j}, A_{k}\right)$ represents the depth of the image pixel at $\mathrm{i}^{\text {th }} \mathrm{C}$ scan, $\mathrm{j}^{\text {th }} \mathrm{B}$-scan, and $\mathrm{k}^{\text {th }} \mathrm{A}$-scan in relation to $\mathrm{R}_{0}$. An example of the obtained $3 \mathrm{D}$ tractographic representation is shown in Fig. 3-2(f). To better visualize the change in fiber orientation, fiber tracts were also displayed using a color map based on the orientation as shown in the color bar in Fig. 3-2(f).

All image processing was implemented using the Matlab software. The open source 3DSlicer (http://www.slicer.org/) was used for 3D data visualization.

\subsection{Results and discussion}

The 3D structure image of the whole mouse heart is shown in Fig. 3-3(a). Fig. 3-3(c) to 33(e) show example B-scan images of the intensity, local retardance and axis extracted along the dashed line in Fig. 3-3(a). Clear optic axis information can be observed at a depth of $1.1 \mathrm{~mm}$ beneath the surface for most parts of the heart tissue, and up to $1.4 \mathrm{~mm}$ at some locations. As an assessment of the overall polarization properties, the average retardance and diattenuation were $2.8 \pm 1.5 \mathrm{rad} / \mathrm{mm}$ and $1.2 \pm 1.1 \mathrm{~mm}^{-1}$ in the left ventricle tissue over an area of $4.0 \times 7.8 \mathrm{~mm}^{2}(\mathrm{~B} \times \mathrm{C})$ from 0.1 to $0.9 \mathrm{~mm}$ beneath the epicardium. The large variation suggested inhomogeneous polarization properties throughout the sample. We also 
noticed that the retardance value in mouse heart was smaller than that obtained in a piece of bovine heart (Fan \& Yao 2013).
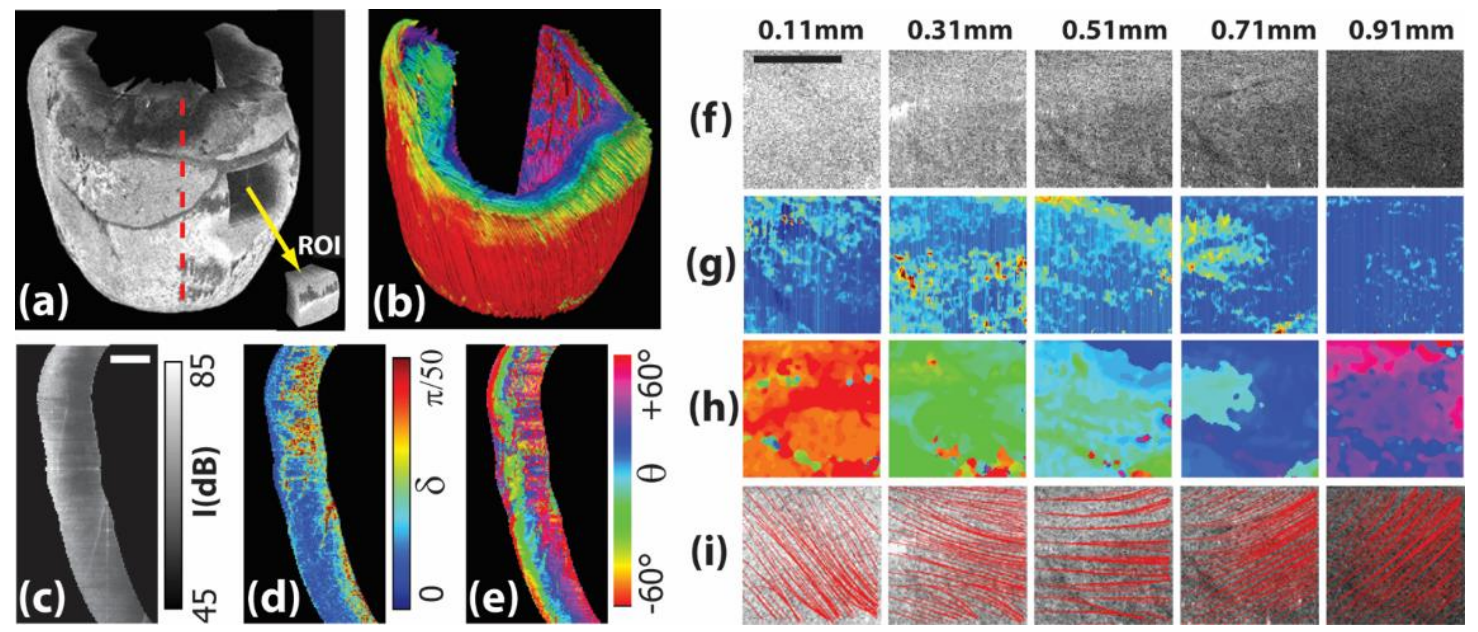

Figure 3-3. The 3D images of a mouse heart. (a) The 3D structure image; (b) the corresponding 3D tractographic visualization. The B-scan images of structure, local retardance and optic axis acquired along the dashed line in (a) are shown in (c)-(e). Also shown are the en face images of the (f) structure, (g) local retardance, (h) local optic axis, and (i) cardiac fiber tract of a small region of interest (ROI) extracted at transmural depths from $0.11 \mathrm{~mm}$ to $0.91 \mathrm{~mm}$. As shown in (a), the ROI was extracted from the lateral side of the left ventricle wall and had a size of $1.57 \mathrm{~mm} \times 20^{\circ}$ rotation $(\mathrm{B} \times \mathrm{C}$-scan). The size bars in (c) and (f) are $1 \mathrm{~mm}$.

The corresponding 3D tractograph is shown in Fig. 3-3(b). Please note that the atrium structure and arteries were removed from the fiber tracking as explained in the previous section. The laminar structure of the fiber orientation can be readily visualized from the color change: red color (negative orientation) at outside surface, and blue (zero degree) to pink color (positive orientation) toward the inner surface. A more detailed examination of a small region of interest (ROI) extracted from the lateral side of the left ventricle is shown in Fig. 3-3(f-i). The ROI had a size of $1.57 \mathrm{~mm}$ along the B-scan and 
covered $20^{\circ}$ rotation along the $\mathrm{C}$-scan $(\sim 1.6 \mathrm{~mm}$ in length). Sample images of the structure and local retardance/optic axis were shown at transmural depths from $0.11 \mathrm{~mm}$ to $0.91 \mathrm{~mm}$. At small depths close to the heart surface the fibrous structures were vaguely recognizable at some locations in the intensity images and showed good agreement with the extracted local optic axis data. The rotation of the fiber orientation with transmural distance from epicardium to endocardium is revealed in Fig. 3-3(i). A “cross-helical” laminar structure is clearly formed by the negatively oriented fibers at $0.11 \mathrm{~mm}$, circumferentially oriented ( $0^{\circ}$ ) fibers at $0.51 \mathrm{~mm}$, and positively oriented fibers at larger depths.

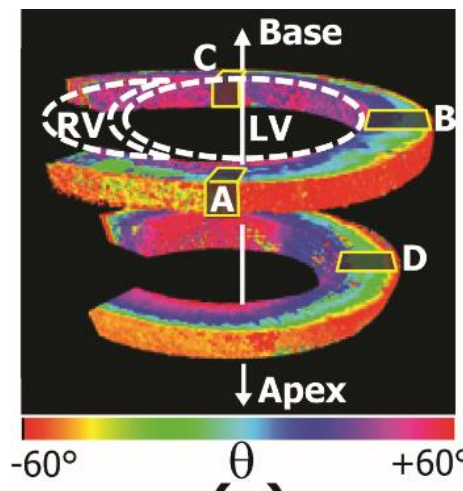

(a)

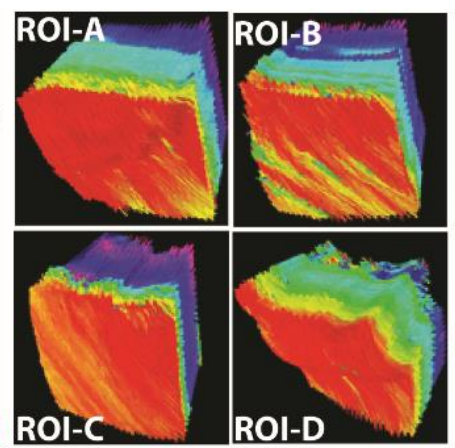

(b)

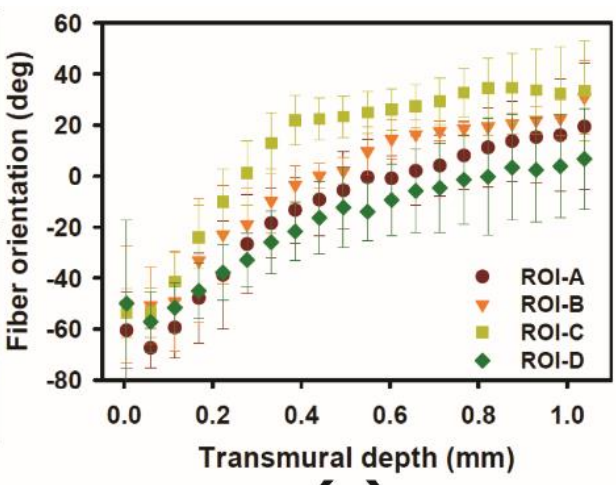

(c)

Figure 3-4. An analysis of the depth dependent fiber orientation changes from selected ROIs. (a) The location of the ROIs. ROI-A, ROI-B, and ROI-C were located around the mid-heart of the left ventricle; while ROI-D was close to the apex of the heart. These ROIs had a size of $0.95 \mathrm{~mm} \times 12^{\circ}$ (B-scan by C-scan). (b) 3D color-coded tractography of the four ROIs. (c) The average fiber orientation as a function of transmural distance from the heart surface. Error bars shown represent standard deviations.

Four additional small pieces of ROIs extracted at various left ventricle locations

(Fig. 3-4) were quantified to examine the organization of myocardial fibers in the mouse heart. Each ROI had a size of $0.95 \mathrm{~mm} \times 12^{\circ}$ rotation (B-scan by $\mathrm{C}$-scan). As shown in 
Fig. 3-4(a), the ROI-A, B, and C were located at the anterior, lateral, and posterior side of the left ventricle, respectively; whereas the ROI-D was located at the apical lateral wall of the left ventricle. Figure 3-4(b) shows the color-coded 3D tractography in the aforementioned four ROIs. A consistent transition in fiber orientation from negative angles (red color) at epicardium to positive angles (blue to pink color) toward endocardium can be seen.

The mean and standard deviation of the fiber orientation in the four aforementioned ROIs were computed and displayed as a function of the transmural depth in Fig. 3-4(c). The overall trend of these results was consistent with those reported in DTI studies (Jiang et al. 2004; Healy et al. 2011). In all four ROIs, the fiber orientation exhibited the classic "cross-helical" structure, i.e. increasing monotonically from negative angles at epicardium to positive angles toward endocardium. The cardiac fibers were orientated at around $-60^{\circ}$ at depths close to epicardium, which is in good agreement with the values reported in both mice (Jiang et al. 2004) and humans (Mekkaoui et al. 2012) using diffusion-tensor magnetic resonance imaging. The depth where the fiber orientation became circumferential $\left(0^{\circ}\right)$ was $0.5 \mathrm{~mm} \sim 0.8 \mathrm{~mm}$ in ROI-A, ROI-B, and ROI-D; where it was $\sim 0.3 \mathrm{~mm}$ in the posterior sector of the left ventricle (ROI-C). In all ROIs, the rate of fiber orientation changing with depth was faster at depths close to the epicardium, which was consistent with previous results obtained in a detailed histology study (Streeter Jr et al. 1969). Quantitatively, the fiber orientation increased with transmural depth at an average rate of $77 \% \mathrm{~mm}, 73 \% \mathrm{~mm}$, and $86 \% \mathrm{~mm}$ at the anterior (ROI-A), lateral (ROI-B), and posterior 
(ROI-C) part of the left ventricle, respectively. These values were similar to those reported in a DTI study of mouse heart (Jiang et al. 2004).

The standard deviation of fiber orientation within these ROIs ranged between $8^{\circ}$ and $20^{\circ}$, which are similar to those previously reported in DTI and histological studies (Karlon et al. 1998; Clayton et al. 2010). In our results, fiber orientation usually had larger variation at depths greater than $0.9 \mathrm{~mm}$ from the epicardium due to degraded signal-tonoise ratio (SNR). Fiber orientation also showed larger variations at locations close to the heart surface. The fiber orientation actually showed a sudden trend change from decreasing to increasing with depth at $\sim 50 \mu \mathrm{m}$ from the outer surface (Fig. 3-4(c)). Similar abrupt changes in fiber orientation were reported previously at locations close to epicardium in large animals such as canines (Clayton et al. 2010). This phenomenon was related to the dense pericardium layer covering the outside wall of the heart. The pericardium layer was portrayed in the structural OCT image as a thin layer with very high reflectance. This

phenomenon was not reported in existing DTI studies of mouse hearts most likely due to the insufficient spatial resolution in existing DTI systems.

\subsection{Conclusion}

In summary, we developed a new scanning and image reconstruction procedure for acquiring tractography in whole mouse hearts. The "cross-helical" laminar structure of myocardial fibers was clearly visualized using this technology. The obtained myocardial 
fiber organization was in accordance with existing knowledge acquired using diffusion tensor magnetic resonance imaging. Although the imaging depth of our current PSOCT system was not able to penetrate the entire heart wall $(1.5-1.8 \mathrm{~mm})$ (Jiang et al. 2004) in mice, it was sufficient to cover the majority of the heart wall from the surface. The imaging depth may be improved by using an incident light at longer wavelengths such as $1060 \mathrm{~nm}$ or $1300 \mathrm{~nm}$. Nevertheless, the micrometer scale resolution achieved by OCT is superior to that obtained in DTI and approaches the capability of histological imaging. Moreover, the fast imaging speed of current OCT technologies far exceeds the speed of DTI and histology analysis. Therefore PSOCT may become a valuable tool for studying myocardial microstructures in small animals. 


\section{Chapter 4. OPT imaging of heart structural remodeling in a mouse model of Duchenne cardiomyopathy}

\subsection{Introduction}

Duchenne cardiomyopathy describes the dilated cardiomyopathy seen in later-stage Duchenne muscular dystrophy (DMD) patients. It is a leading cause of morbidity and mortality in DMD patients (Duan 2006). The mdx mouse is one of the most commonly used DMD animal model where the dystrophin expression is blocked by a point mutation (Duan 2006). Mdx mice develop a progressive cardiomyopathy that shares many features with the human disease (Duan 2006). Although mdx mice have been studied extensively, the heart structural remodeling in this model has not been investigated in detail (Cheng et al. 2012). The heart is a highly adaptive organ and its structure may change in response to progressive deterioration of cardiac function. It is known that the myofibers in a healthy heart are organized in an exquisite and optimized structure (Streeter Jr et al. 1969), which was often altered in heart diseases as part of the pathological remodeling process (Helm et al. 2006; Benoist et al. 2012). The knowledge of detailed myocardial fiber changes during disease progression is essential for understanding the intricate structure-function

\footnotetext{
* This chapter was from a published article: Wang, Y., Zhang, K., Duan, D. and Yao, G., 2017. Heart structural remodeling in a mouse model of Duchenne cardiomyopathy revealed using optical polarization tractography. Biomedical Optics Express, 8(3), pp.1271-1276.
} 
relationship in heart diseases.

The MRI based diffusion-tensor imaging (DTI) technology is the only practically available tool for imaging myocardial fiber structure in the whole heart (Mekkaoui et al. 2015). However, in addition to its high costs, the limited spatial resolution of DTI makes it extremely challenging for revealing detailed myocardial fiber structure in the small mouse hearts. A typical mouse heart has a diameter of $\sim 6 \mathrm{~mm}$ with $1 \sim 2 \mathrm{~mm}$ thick ventricular wall (Vetter et al. 2005). The myofiber orientation can change over $100^{\circ}$ across the thin ventricular wall (Wang \& Yao 2013; Mekkaoui et al. 2015). Abrupt orientation changes of more than $50^{\circ}$ were observed between different epicardial layers (Wang \& Yao 2013). Therefore, a sufficient resolution is important for correctly imaging detailed fiber orientation changes in the ventricular wall of the heart.

Optical polarization tractography (OPT) is a newly developed method that can image high-resolution fiber organization in fibrous tissues (Wang et al. 2014; Azinfar et al. 2015; Wang et al. 2015b). It was based on the Jones matrix implementation of the polarization-sensitive optical coherence tomography (PSOCT). OPT uses Jones calculus to construct the depth resolved optic axis for measuring fiber orientation with microscopic resolution (Fan \& Yao 2012a). In this study, OPT was applied to image the myocardial fiber structures in freshly excised whole mouse hearts. The results revealed significant structural remodeling in the mdx mouse model of Duchenne cardiomyopathy.

\subsection{Methods}




\subsubsection{OPT system.}

The OPT method was implemented in a $0.85 \mu \mathrm{m}$ wavelength spectral-domain Jones matrix PSOCT system which has been described in detail previously (Fan \& Yao 2013; Wang et al. 2016). Briefly, the system measures pixel-wise Jones matrix of the sample by using incident light with alternating right- and left-circular polarizations. For each incident polarization, the horizontally and vertically polarized components of the interference signals were detected using a custom spectrometer equipped with a 1024-pixel line scan camera running at 50k A-lines/sec. An iterative Jones calculus based algorithm was applied to extract the depth-resolved optic axis for mapping the fiber orientation (Wang et al. 2016). The fiber orientation measured is between $\left[-90^{\circ}, 90^{\circ}\right]$ and represents the projected orientations within the plane perpendicular to the incident light (Ciszek et al. 2007; Wang et al. 2015b), which is analog to the "helix" angle measured in DTI (Vetter et al. 2005; Cheng et al. 2012). A galvanometer was used with a telecentric lens (36 mm focal length) to scan $8 \mathrm{~mm}$ in each B-scan. The resolution was $12.4 \mu \mathrm{m}$ at the focus in the lateral direction and $5.9 \mu \mathrm{m}$ in the axial direction in tissue.

\subsubsection{OPT imaging of a whole mouse heart.}

All animal experiments were approved by the institutional animal care and use committee. The freshly excised hearts from six 7-mo old male mdx4cv mice (the "mdx" group) and six age-matched male C57BL/6ScNJ mice (the "BL6" group) were imaged using the OPT system. The procedure to image the whole heart tractography were described in Ch.2. 
Briefly, the heart was mounted on a rotational stage via a 20-gauge needle passing through the long axis of the heart (between the apex and center of the base). The needle was fixed on the base of the stage and was aligned with the rotational axis. During imaging, the stage was rotated continuously over $360^{\circ}$ at a speed of $1.25^{\circ} / \mathrm{sec}$ while the incident light repeatedly scanned along the long axis of the heart (as the B-scan). Each B-scan covered 8 mm with 2000 A-scans. A total of 3600 B-scans were acquired at a recording speed of 12.5 B-scans/s. It took 288 seconds to image a whole heart. The heart diameter was measured using a caliper.

The constructed 3D dataset of fiber orientation had $280 \times 2000 \times 3600$ pixels (in $\mathrm{A} \times \mathrm{B} \times \mathrm{C}$ scans) and covered a corresponding imaging area of $1.1 \mathrm{~mm} \times 8.0 \mathrm{~mm} \times 360^{\circ}$. The 2D "planar" tractography was first built at each en faces plane using Matlab streamline functions (Wang \& Yao 2013). A 5×5 (pixels) median filter was applied to all processed B-scan images to improve signal-to-noise. The stack of the "planar" 2D tractography was then polar-transformed into the 3D coordinates from the measured heart diameter and heart surface as described previously (Wang \& Yao 2013). The OPT results were visualized using the 3DSlicer software (www.slicer.org).

\subsection{Results \& Discussion}

Figure 4-1(a) shows intensity images of a BL6 and an mdx mouse heart. The BL6 heart appeared in a pear-like shape; whereas the mdx heart was bigger and close to a cylindrical 
shape. In all hearts tested, the mdx heart had an average diameter of $6.88 \pm 0.48 \mathrm{~mm}$ which was significantly larger ( $p=0.013$, Student's t-test) than the $6.15 \pm 0.25 \mathrm{~mm}$ diameter of the BL6 heart.

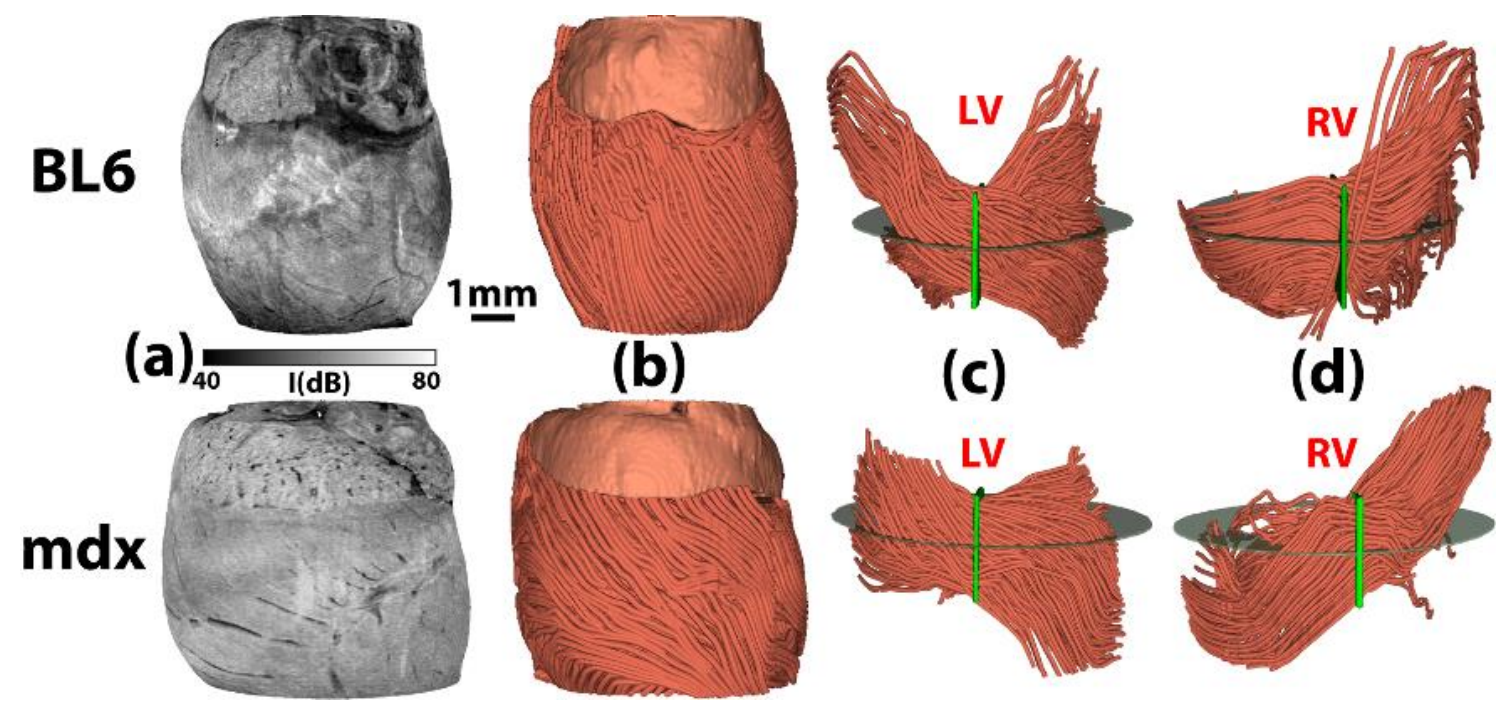

Figure 4-1. 3D OPT images of whole mouse hearts. (a) Intensity images of a BL6 heart and an mdx heart. (b) The corresponding 3D tractography of the BL6 heart and the mdx heart, both with the left ventricle (LV) facing front. Also shown are depth-resolved tractography showing myofibers passing through a $1.5 \mathrm{~mm}$ high region of interest (green plate) across (c) the LV and (d) the right ventricle (RV) of the two hearts.

Figure 4-1(b) illustrates the global ventricular tractography of the same two hearts shown in Fig. 4-1(a). Since no clear optic axis data was obtained in the atrial and aorta tissues, they were shown in solid color. The fiber tractography shown in Fig. 4-1(b) was obtained at the $100 \mu \mathrm{m}$ depth beneath the epicardium. The associated animations revealed the change of the fiber orientation of the entire heart. Overall, the fiber orientation appeared similar in the left ventricle (LV) of both the BL6 and mdx hearts, but quite different in the right ventricle $(\mathrm{RV})$. To examine depth-resolved fiber orientation across the ventricular 
wall, Figures 4-1(c) and 4-1(d) showed myocardial fibers that pass through a $1.5 \mathrm{~mm}$ high (along the B-scan) region of interest (ROI) located at the center of the LV and RV, respectively. The associated animations show the change of myofiber structure from the epicardium to the endocardium.

In both strains, the LV showed the classic "cross-helical" profile (Streeter Jr et al. 1969) (Fig. 4-1(c)). "Negatively orientated" fibers at the epicardium transitioned to circumferential at the middle of the ventricular wall, and became positively oriented toward the endocardium. However, the mdx heart appeared to have smaller helix angles and a smaller rate of orientation change over the LV wall than the BL6 heart. The myofibers in the BL6 RV had much less orientation changes across the heart wall than in the LV. The BL6 heart showed some "vertical" fibers close to the RV surface at the proximity of the right ventricle vein (RCV) (Agger et al. 2016). This was not observed in the mdx heart. The RV of the mdx heart appeared to have more variations in fiber organization in the en face plane than the BL6 heart.

We further quantitatively analyzed the depth-resolved fiber organization in both the BL6 and mdx hearts. To compensate for the variation in heart structure among different hearts, an evaluation "window" was defined in both the LV and RV based on common structural features including cardiac veins (Agger et al. 2016) observed in the intensity images of the mouse hearts (Fig. 4-2(a)). The vertical yellow dashed line was first drawn along the B-scan direction from the lowest boundary of the left atrium. This line ended at the intersection with the left cardiac vein (LCV) (Ciszek et al. 2007; Agger et al. 2016) . 
The middle point of this line was then identified as the center of the LV window ("Lc" in Fig. 4-2(a)). In addition, the white dashed line was drawn from Lc along the C-scan direction. The intersection of this line with the RCV was selected as the center of the RV window ("Rc" in Fig. 4-2(a)). The two evaluation windows were then created by extending from their corresponding centers to reach a size of $2.8 \mathrm{~mm} \times 70^{\circ}(700 \times 700$ pixels, $\mathrm{B} \times \mathrm{C})$ as illustrated as the blue boxes in Fig. 4-2(a). These windows were manually determined in all hearts and their coordinates were recorded for subsequent calculations.
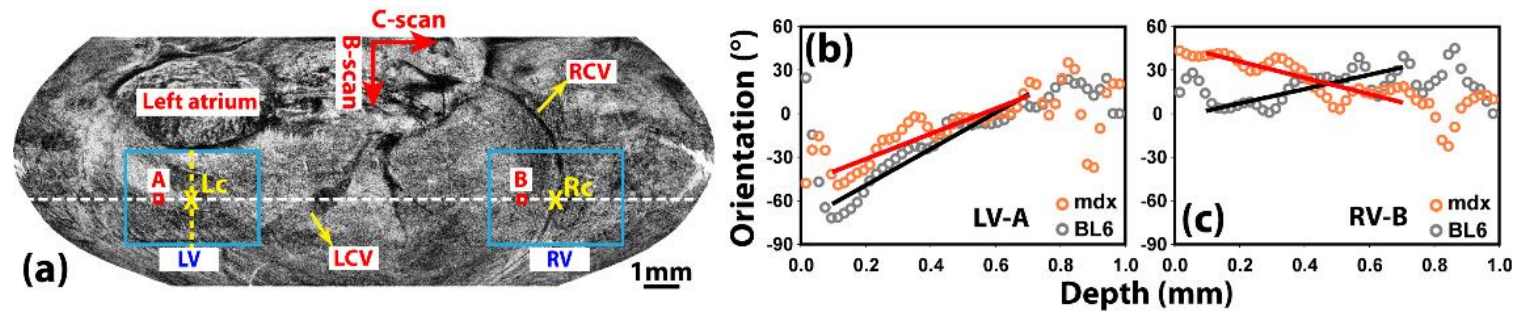

Figure 4-2. (a) An illustration of the "window" in LV and RV (blue boxes) for quantitative analysis. LCV: left cardiac vein; RCV: right cardiac vein (Ciszek et al. 2007). Also shown are example depth profiles of myocardial fiber orientation calculated at ROIs at (b) LV and (c) RV as labeled in red boxes in (a). These sample data were obtained from the same hearts shown in Fig. 4-1.

Figures 4-2(b) and 4-2(c) show representative depth profiles of the fiber orientation at representative region-of-interests (ROIs) inside the LV and RV, respectively. The ROIs were marked as red boxes on the white dashed line in Fig. 4-2(a). The ROI size was 0.1 $\mathrm{mm} \times 2.5^{\circ}(25 \times 25$ pixels in B-scan $\times$ C-rotation $)$. The fiber orientation values were obtained using circular averaging within this evaluation window (Azinfar et al. 2015). Consistent with the global tractography in Fig. 4-1, the depth-profiles of the fiber orientation in the LV showed the "cross-helical" transition from negative to positive 
orientation in both the BL6 and mdx hearts. An "inversed" transition (from positive to negative orientations) was observed in the BL6 heart within $100 \mu \mathrm{m}$ from the epicardium; but this was not clear in the mdx heart (Fig. 4-2(b)). Such an inverse trend was also observed in our previous studies (Wang \& Yao 2013) and reported in a recent high resolution (9.4-T) DTI study of the larger ovine heart (Meyers \& Townsend 2015). This was not reported in DTI studies of healthy mouse hearts (Sosnovik et al. 2009a), likely due to insufficient resolution. The fiber orientation of the BL6 RV was only slightly positive with very small changes across the ventricular wall. However, the mdx heart showed significant "negative" orientation changes over the depth.

Linear fitting was applied to calculate the "slope" of the orientation change between 0.1 and $0.7 \mathrm{~mm}$ in depth. Orientation data within the $0.1 \mathrm{~mm}$ from the heart surface were excluded in the fitting to avoid the "inverse" trend shown in Fig. 4-2(b). The fitted slopes were $124.9^{\circ} / \mathrm{mm}\left(\mathrm{R}^{2}=0.91\right.$ and root-mean-square-error $\left.(\mathrm{RMSE})=7.0^{\circ}\right)$ in the BL6 LV and $86.8^{\circ} / \mathrm{mm}\left(\mathrm{R}^{2}=0.81\right.$ and $\left.\mathrm{RMSE}=7.4^{\circ}\right)$ in the $\mathrm{mdx} \mathrm{LV}$. For the RV curves (Fig. 4-2(c)), the obtained slopes were $49.5^{\circ} / \mathrm{mm}\left(\mathrm{R}^{2}=0.61\right.$ and $\left.\mathrm{RMSE}=6.8^{\circ}\right)$ in the BL6 heart and $56.5^{\circ} / \mathrm{mm}\left(\mathrm{R}^{2}=0.70\right.$ and $\left.\mathrm{RMSE}=6.5^{\circ}\right)$ in the $\mathrm{mdx}$ heart.

The "slope" of the fiber orientation change with depth was calculated for all the ROIs of the same size $\left(0.1 \mathrm{~mm} \times 2.5^{\circ}\right)$ within the entire $\mathrm{LV}$ and $\mathrm{RV}$ evaluation windows. In other words, the depth profiles similar to those shown in Figs. 4-2(b) and 4-2(c) were constructed and linearly fitted for a total of $28 \times 28=784$ ROIs inside the evaluation window. 

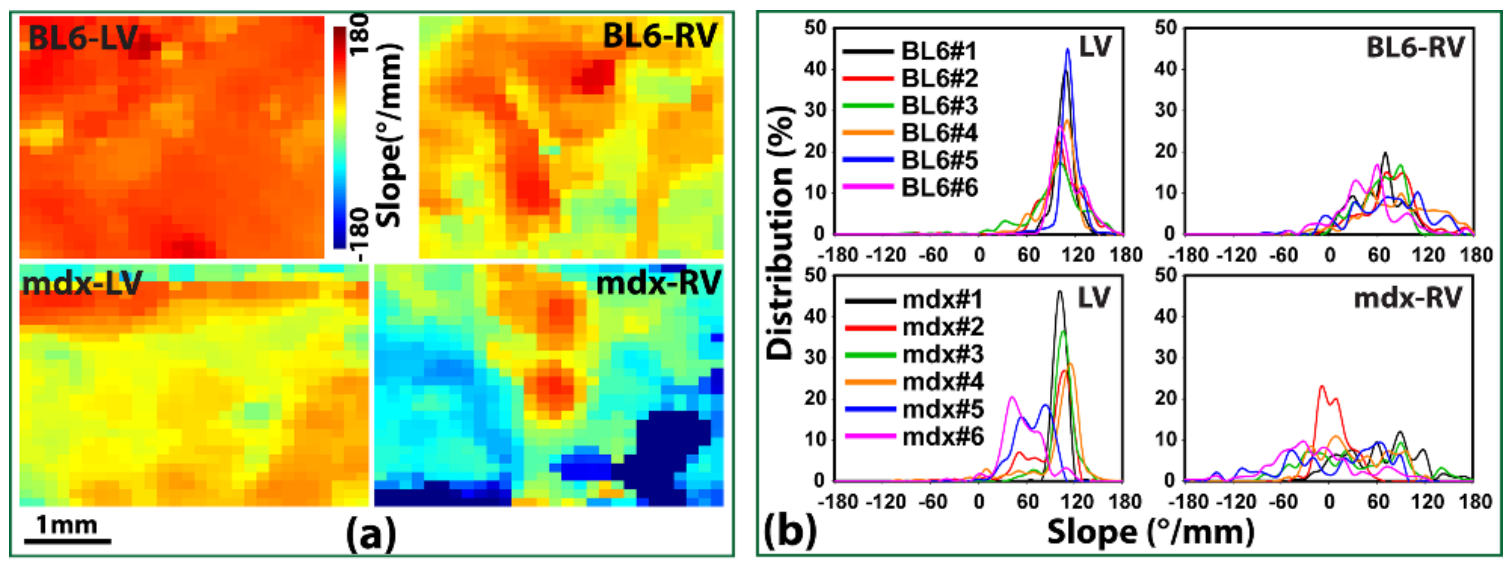

Figure 4-3. (a) Examples of color-coded "slopes" of fiber orientation change with depth in the LV and RV of the same hearts shown in Fig. 4-2. (b) Distributions of the fiber orientation slopes obtained in all six BL6 hearts and six mdx hearts within the same LV and RV evaluation windows as defined in Fig. 4-2(a). BL6\#1 and mdx\#6 were the mice used in (a).

The slope values can be color-coded for visualization (Fig. 4-3(a)). The image width of the mdx heart was wider along the $\mathrm{C}$-scan direction because the mdx heart had a bigger diameter. More than $92.9 \%$ of the $\mathrm{LV}$ regions in both hearts had fitting $\mathrm{R}^{2}>0.6$ or RMSE $<10^{\circ}$. The slopes were relatively homogeneous in the LV of both the mdx and BL6 hearts; but the values were overall smaller in the mdx heart than the BL6 heart. The depth profiles in the RV showed more location-dependent variations in both hearts. However, the variations in the mdx heart were bigger. Only $68.8 \%$ of the $\mathrm{mdx} R \mathrm{R}$ and $84.9 \%$ of the BL6 $\mathrm{RV}$ had fitting $\mathrm{R}^{2}>0.6$ or RMSE $<10^{\circ}$. Poorer linear fitting in the $\mathrm{RV}$ was also reported in previous DTI studies (Benoist et al. 2012). The fitting in the RV was also affected by the presence of the RCV as more abrupt orientation changes were observed at the proximity of the RCV.

Figure 4-3(b) shows the distributions of the orientation slopes obtained in all 
experimental animals. The regions with fitting $\mathrm{R}^{2}<0.6$ and $\mathrm{RMSE}>10^{\circ}$ were not used in the calculation. Overall, the linear fitting in the $\mathrm{mdx}$ heart had $\mathrm{R}^{2}=0.81 \pm 0.05$ and $\mathrm{RMSE}=8.19^{\circ} \pm 1.54^{\circ}$ in the $\mathrm{LV}$, and $\mathrm{R}^{2}=0.64 \pm 0.10$ and $\mathrm{RMSE}=8.99^{\circ} \pm 1.28^{\circ}$ in the $\mathrm{RV}$. These fitting performance was similar to those of the BL6 heart: $\mathrm{R}^{2}=0.82 \pm 0.03$ and $\mathrm{RMSE}=8.25^{\circ} \pm 0.47^{\circ}$ in the $\mathrm{LV}$, and $\mathrm{R}^{2}=0.68 \pm 0.08$ and $\mathrm{RMSE}=8.51^{\circ} \pm 1.91^{\circ}$ in the $\mathrm{RV}$. The distributions in the BL6 LV were highly consistent and the rate of orientation change was $100 \sim 110^{\circ} / \mathrm{mm}$ at the peak position of the distribution. The distribution in the $\mathrm{mdx} \mathrm{LV}$ varied among six mice with three hearts showing similar distributions as that of the BL6 heart; whereas the other three had broader distributions and a shift toward smaller slope values. Although the distributions in the RV were generally broader than that in the LV, the BL6 hearts still showed quite consistent distributions with positive slope values in more than $96.2 \%$ of the RV regions. However, the mdx RV showed much broader distributions with $38.8 \%$ of the mdx RV had negative slope values $\left(-38.3 \pm 34.0^{\circ} / \mathrm{mm}\right.$ in average).

Figure 4-4 shows the group comparison of the mean slope of the orientation change over depth obtained in all hearts. All the hearts showed "positive" slopes in the LV. The mdx group showed smaller slope values $\left(86.4^{\circ} / \mathrm{mm} \pm 20.4^{\circ} / \mathrm{mm}\right)$ than the BL6 group $\left(104.4^{\circ} / \mathrm{mm} \pm 7.0^{\circ} / \mathrm{mm}\right)$ in the $\mathrm{LV}$; but the difference did not reach statistical significance ( $p=0.085$, Student's t-test). However, the Levene's test indicated that the mdx group had a bigger variance in the slope data of the $\operatorname{LV}(p=0.018)$. The group difference was more drastic in the RV where the variance was similar in both groups ( $p=0.075$, Levene's test). The group average of the slope was significantly smaller ( $p=0.004$, Student's t-test) in the 
mdx RV $(20.3 \pm 28.4 \% \mathrm{~mm})$ than in the BL6 RV $(67.9 \pm 12.6 \% \mathrm{~mm})$.

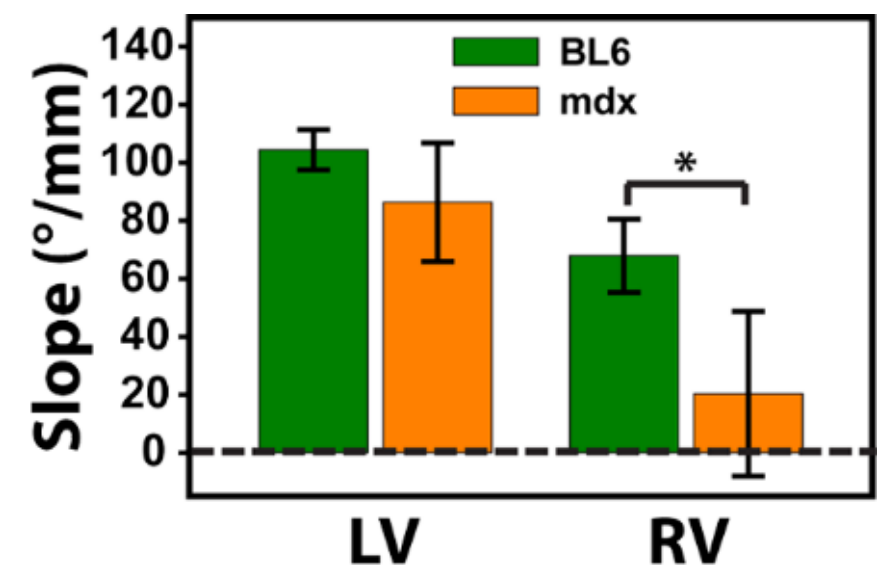

Figure 4-4. A group comparison of the average rate of fiber orientation change with depth in the LV and RV of the six BL6 mice and six mdx mice. * $p<0.01$.

The severe structural remodeling observed in the RV of the 7-month-old mdx mouse is likely caused by the diaphragm degeneration related pulmonary dysfunction (Stuckey et al. 2012; Barbin et al. 2016). Deterioration of the respiratory function increases pulmonary vascular resistance which then leads to increased RV stress (Barbin et al. 2016). This process eventually results in tissue damage in the RV (Stuckey et al. 2012; Barbin et al. 2016). The global heart structural remodeling is most likely a direct consequence of the myofiber damage in the RV. The large between-animal variations of the fiber organization observed in the LV of the mdx model may be indications of different disease progression. More systematic studies are warranted to clarify the pathological process leading from local cardiac muscle damage to changes in the global heart structure.

The $\sim 1.0 \mathrm{~mm}$ imaging depth achieved in this study was mainly limited by the 0.85 $\mu \mathrm{m}$ light source. The ventricular wall thickness of the 7-mo mouse heart was $\sim 1.0 \mathrm{~mm}$ 
(Stuckey et al. 2012) and the RV wall is thinner than the LV wall. Therefore the $1.0 \mathrm{~mm}$ depth may not be sufficient to image through the entire LV wall. The imaging depth in heart can be improved by using a longer wavelength (e.g. $1.3 \mu \mathrm{m})$. The mdx hearts have similar ventricular wall thickness as the controls before 9-month of age (Stuckey et al. 2012). Therefore the different slopes in fiber orientation changes cannot be caused from any differences in the wall thickness. It is important to note that this study only measured the helix angle of the 3D myocardial fiber located within the plane perpendicular to the incident light (Cheng et al. 2012; Wang \& Yao 2013). Therefore, any orientation changes in planes that are parallel to the incident light were not detected. Imaging the true 3D fiber orientation is necessary to fully characterize the fiber structure changes, which can be realized using a variable-incident angle implementation of the OPT (Wang et al. 2016).

\subsection{Conclusions}

The OPT technology was applied to investigate the structural remodeling in the hearts of an mdx mouse model of the Duchenne cardiomyopathy. OPT revealed significantly altered myocardial fiber structure in the hearts of 7-mo old mdx mice in particular in the right ventricle. This study demonstrated the potential of OPT as a useful imaging technique for studying high-resolution heart structural remodeling in small animal models of heart diseases. 


\section{Chapter 5. Imaging microscopic cardiac damage in mdx mice using OPT}

\subsection{Introduction}

Changes in heart structure are important pathological features in heart diseases (Cohn et al. 2000). The myofibers in healthy heart are organized in an exquisite helical-like structure (Weber 1989), which enables normal electric signal propagation and a coordinated mechanical force production for efficient blood pumping (Streeter Jr et al. 1969; Taccardi et al. 1994; Gilbert et al. 2007). Action potential propagates 2 to 10 times faster along the heart muscle fibers than along the transverse direction (Kléber \& Rudy 2004). Disruption of normal myofiber architecture in the heart contributes to numerous cardiac diseases such as in the cases of hypertrophic and dilated cardiomyopathy (Fenton \& Karma 1998; Helm et al. 2006; Tseng et al. 2006; Strijkers et al. 2009).

Duchenne muscular dystrophy (DMD) is the most common lethal childhood muscle disease (Alan E. H. Emery 2003), and is caused by the null mutations of the dystrophin gene. Duchenne cardiomyopathy refers to the dilated cardiomyopathy seen in later-stage DMD patients (Duan 2006; Shin et al. 2010). It is a leading cause of morbidity and mortality in DMD (Valentine et al. 1989; Nigro et al. 1990). The $m d x$ mouse, where the dystrophin expression is blocked by a point mutation (Sicinski et al. 1989; Lee et al. 2012), is the most commonly used DMD animal model (Valentine et al. 1989; Nigro et al. 1990), 
developing a progressive cardiomyopathy that shares many features with human disease (Valentine et al. 1989; Nigro et al. 1990; Spurney 2011). Histology studies have revealed many pathological changes in $m d x$ mice including absent dystrophin at sarcolemma (Kido et al. 2004), increased fibrosis at right ventricle (RV) with ages for young mdx mice (Meyers \& Townsend 2015), and exacerbated inflammatory fibrosis in heart (11-monthold, two months exercise) (Barbin et al. 2016). In addition, abnormal electrocardiogram (ECG) was observed in $m d x$ mice including increased heart rate, QRS duration, QT interval, and Q wave, and decreased PR interval (Bostick et al. 2008a; Bostick et al. 2008b; Bostick et al. 2009). Echocardiography revealed that the mdx mice had an enlarged posterior wall thickness at 10-month-old and a greater left ventricle (LV) mass at 12month-old (Fayssoil et al. 2013). Myocardial energetic deficits have been reported in $m d x$ mice at young age (5-month-old) using magnetic resonance spectroscopy (Cui et al. 2015). Imaging the myocardial structure is vital to elucidate the pathogenic mechanisms and evaluate treatment outcomes. MRI methods such as diffusion-tensor MRI (DTI) and diffusion spectrum MRI have achieved great success in imaging myofiber orientation (Sosnovik et al. 2009a; Nielles-Vallespin et al. 2013; Sosnovik et al. 2014). Different helix slopes at RV were reported in rats (Benoist et al. 2012) and altered angulations of myocytes were observed in lambs (Agger et al. 2016) in studying of the pulmonary hypertension. Reduced degree of helix angle at LV were reported in studying of the cardiac myosin binding protein-C using transgenic mice (Taylor et al. 2016). However, few work previously reported on imaging the myofiber architecture change in the dystrophin- 
deficient hearts except for a DTI study of sheet angle difference in hearts (Cheng et al. 2012).

Because both cellular- and global-level changes are involved in cardiac remodeling, it is important to thoroughly evaluate heart structures. Cellular-level structural changes (such as fiber branching and disarray) have important pathological significance especially at early stages of myocardial diseases (Karlon et al. 1998; Sasaoka et al. 2003; Niederer et al. 2004). Unfortunately, none of the existing technologies has the capability to imaging the global myocardial structure at cellular level resolution.

Traditionally, histological staining is used to study myofiber orientation in the heart (Streeter \& Bassett 1966; Streeter Jr et al. 1969). Using this approach, Streeter et al. established the well-known "cross-helical" structure of myocardial fibers across the ventricular wall (LeGrice et al. 1995a; Vetter \& McCulloch 1998). However, histological examination of serial heart sections is labor-intensive and only practical for imaging a very small area and cannot accurately assess the global myocardial architecture (Vetter \& McCulloch 1998). The spatial resolution of MRI based methods is limited to 100 200 $\mu \mathrm{m}$ or worse (Jiang et al. 2004; Healy et al. 2011), which makes it impossible to reveal important cellular details. Ultrasound technologies are also explored for mapping myocardial fiber orientation (Lee et al. 2012; Papadacci et al. 2014). But their reported resolution is no better than that of MRI. Therefore neither MRI nor ultrasound can be used to study the global myofiber architecture at the cellular level. A sufficiently high resolution becomes more important for imaging the heart of small animals such as mice which are the 
most commonly used animal models due to their accessibility and low cost (Doevendans et al. 1998; Breckenridge 2010). Genetic modifications are widely used to produce mouse

models to simulate human heart diseases (Hewing \& Fisher 2012). Because of the ventricular wall in a typical mouse heart is only $1 \sim 2 \mathrm{~mm}$ thick, a spatial resolution worse than $100 \mu \mathrm{m}$ is unable to reveal the detailed myofiber architecture in the ventricular wall.

A recent imaging technology "optical polarization tractography" (OPT) which is developed based on PSOCT (Fan \& Yao 2012c, b, 2013), can visualize myocardial fiber architecture with histology-like accuracy (Wang et al. 2014). Previously, OPT has been used to reveal the classic fiber structure in ventricles (Wang \& Yao 2013) and the architecture remodeling in dystrophic hearts (Wang et al. 2017). Fiber disarray in heart or myocyte disarray (Pirolo et al. 1982) has been reported in many heart diseases including in infarcted rodent hearts (Strijkers et al. 2009; Sosnovik et al. 2014; Sosnovik et al. 2009b), hypertrophic cardiomyopathy in human (Tseng et al. 2006) and in mice (Wang et al. 2010). Here, we applied OPT study cellular level myocardial fiber disarray in mdx heart and to visualize the $3 \mathrm{D}$ damage in heart.

\subsection{Methods}

\subsubsection{OPT system.}

A single-camera spectral domain Jones matrix based PSOCT system (Fan \& Yao 2010b) was used to acquire OPT images. A superluminescent diode (SLD) $\left(\lambda_{0}=847.8 \mathrm{~nm}, \Delta \lambda=\right.$ 
$58.3 \mathrm{~nm}$ ) is used as the light source. A linear polarizer converts the incident light into a linearly polarized light. The polarization state of the incident light was then modulated between right- and left-circular polarizations using an electric optical modulator (Fan \& Yao 2010b). The modulated light is then split into the sample arm and the reference arm. In the reference arm, a polarization-sensitive beam splitter splits the light into horizontally (H) and vertically (V) polarized light. Two separate mirrors then then reflect the light back. At the sample arm, a 5×, f36 mm telecentric scan lens (LSM03-BB, Thorlabs, Newton, NJ) was used as the imaging objective. A $2 \mathrm{D}$ galvanometer scanner was used for sample scanning which covered an area of $8 \times 8 \mathrm{~mm}^{2}$ on the sample surface. At the detection arm, a custom spectrometer equipped with a 1024-pixel line scan camera (AVIIVA SM2, e2v, Milpitas, CA) was designed to detect the interference signal and the images acquisition speed was 50k A-lines/sec. This imaging system had a measured lateral resolution of 12.4 $\mu \mathrm{m}$ and a measured axial resolution of $5.9 \mu \mathrm{m}$ in tissue within a $1.5 \mathrm{~mm}$ imaging depth.

\subsubsection{Samples and scanning procedure.}

Six 7-mo-old mdx4cv mice (labeling as "mdx" group) and six age-matched C57BL/6 mice (labeling as "BL6" group) were used in this study. The hearts were excised and imaged immediately after mice euthanasia. A rotation-scanning procedure was developed to image the whole heart in our previous study (Wang \& Yao 2013). A 20-gauge needle was mounted on a rotational stage (PRM1Z8, Thorlabs, Inc., Newton, New Jersey, USA) along the rotational axis, and the heart sample was hold on the needle by passing through heart apex and center of the base. The stage was rotated continuously over $360^{\circ}$ at a speed of 
$1.25 \%$ sec to cover the entire heart. A total of 3600 B-scans (2000 A-lines in each B-scan covering $8 \mathrm{~mm}$ ) were acquired at a speed of $12.5 \mathrm{~B}$-scans/s to complete an entire heart scanning, and it took 288 seconds. In this imaging geometry, the incident light was aligned with Z-axis (A-scan), and B-scan was aligned with $\mathrm{Y}$-axis which is in parallel to the rotational axis. The measured fiber orientation $\theta$ from OPT had range of $\left[-90^{\circ}, 90^{\circ}\right]$, were projected myocardial fiber orientations within XY plane.

After OPT imaging, two mdx4cv hearts and two BL6 hearts were embedded in optimal cutting compound and frozen in liquid nitrogen. For histology processing, each histological slice had thickness of $10 \mu \mathrm{m}$ sectioned from the tissue block. Four successive histology slices with a step of $80 \mu \mathrm{m}$ in each heart were selected and stained with hematoxylin and eosin (HE) to study the myofiber damage. Dystrophin was detected in addition histology slices using a 1st monoclonal antibody (MANDYS8, Sigma, Saint Louis Missouri, USA) and a 2nd antibody (Alexa fluor $594 \mathrm{~F}(\mathrm{ab}) 2$ fragment of goat anti-mouse $\lg \mathrm{G}(\mathrm{H}+\mathrm{L})$, Invitrogen, Rockford, Illinois, USA). Histological images were acquired using a Nikon Eclipse E800 microscope equipped with a QImaging RETIGA 1300 camera.

\subsubsection{Imaging process.}

The entire 3D OPT dataset had a total $280 \times 2000 \times 3600$ pixels along the A-, B- and C-scan directions and covered an imaging area of $1.1 \mathrm{~mm} \times 8.0 \mathrm{~mm} \times 360^{\circ}(\mathrm{A} \times \mathrm{B} \times \mathrm{C})$. The physical scanning distance at then sample surface in C-scan changes with the heart diameters and surface geometric shape. The local depth-resolved polarization properties including retardance, (slow) optic axis, and diattenuation were computed from the 3D dataset of 
Jones matrix at each image pixel. The retardance is defined as $\delta=2 \pi \mathrm{d} \Delta \mathrm{n} / \lambda$. Where $\Delta \mathrm{n}$ is the "birefringence", denoting the difference in sample refractive indices along the orthogonal fast and slow optic axes; and $d$ is the physical pathlength of the light in tissue. The birefringence can be also derived from retardance as $\Delta \mathrm{n}=\delta \lambda /(2 \pi \mathrm{d})$. The optic axis $\theta$ in our results was the "slow" optic axis representing the muscle fiber orientation in the en face (B-C) plane (Fan \& Yao 2012b). The diattenuation is defined as the different optical attenuation coefficient along the fast and slow optic axes. The local retardance and diattenuation were first calculated using similar matrix transformation (Fan \& Yao 2012b). Then a Jones calculus based iteration algorithm was applied to extract depth-resolved optic axis (Fan \& Yao 2012c, b, 2013).

The procedure to construct the heart tractography has been described in our previous study (Wang \& Yao 2013). To explore the potential muscle damage in the ventricles, the circular standard deviation (Karlon et al. 1998) of the fiber orientation was used in this study to identify regions with potential muscle damage:

$$
\Delta \theta=\frac{\sqrt{-2 \ln \left(\sqrt{\left\langle\cos 2 \theta_{p}\right\rangle^{2}+\left\langle\sin 2 \theta_{p}\right\rangle^{2}}\right)}}{2},
$$

where $\theta_{\mathrm{p}}$ indicates the local fiber orientation obtained in OPT, and " $<>$ " denotes the averaged value within an evaluation window. However, $\Delta \theta$ cannot be used as a single method to segment the muscle damage out of the normal muscle fibers because the fiber orientations in heart are much complicated and areas with actual fiber transitions such as interventricular walls could be incorrectly identified as tissue damage. Entropy (E), a 
method to detect the image randomness, was also applied in this study as another noise indicator to identify potential muscle injury:

$$
E=-\sum\left[P \cdot \log _{2}(P)\right]
$$

where $\mathrm{P}$ is the histogram count at different gray-scale level of the local fiber orientation $\theta_{\mathrm{p}}$ within the same sized evaluation window. The evaluation window for computation of $\Delta \theta$ and entropy was $100 \mu \mathrm{m} \times 2.5^{\circ}(\mathrm{B}$-scan $\times \mathrm{C}$-scan $)$ at each depth in the $3 \mathrm{D}$ OPT dataset.

Thresholding-based segmentations were applied on $\Delta \theta$ and $\mathrm{E}$ using a threshold of $45.0^{\circ}$ for $\Delta \theta$ and 0.8 for entropy. The commonly segmented areas were identified as muscle damage. Damage ratio was then quantified using the percent of damaged pixels. Because this damage ratio was not normally distributed among all animals, the KolmogorovSmirnov (K-S) test was used to evaluate the group difference between BL6 hearts and $m d x$ hearts. All image processing was implemented using Matlab.

\subsection{Results}

An evaluation area with a size of $2.8 \mathrm{~mm} \times 70^{\circ}$ (B-scan $\times$ C-rotation) was placed at $\mathrm{LV}$ and RV in every heart. The locations were described in our previous study (Wang et al. 2017). Each evaluation window was sub-divided into $28 \times 28$ small ROIs, and each ROI had size of $0.1 \mathrm{~mm} \times 2.5^{\circ}(\mathrm{B}$-scan $\times$ C-rotation $)$.

Figure 5-1(a) showed example 2D birefringence $(\Delta \mathrm{n})$ images at depth of $300 \mu \mathrm{m}$ in two sample hearts. Figure 5-1(b) showed the distributions of birefringence in these 
evaluation windows from all the hearts. The birefringence in $m d x$ hearts showed more heart-dependent distributions than that in BL6, but the average birefringence showed no overall group difference.
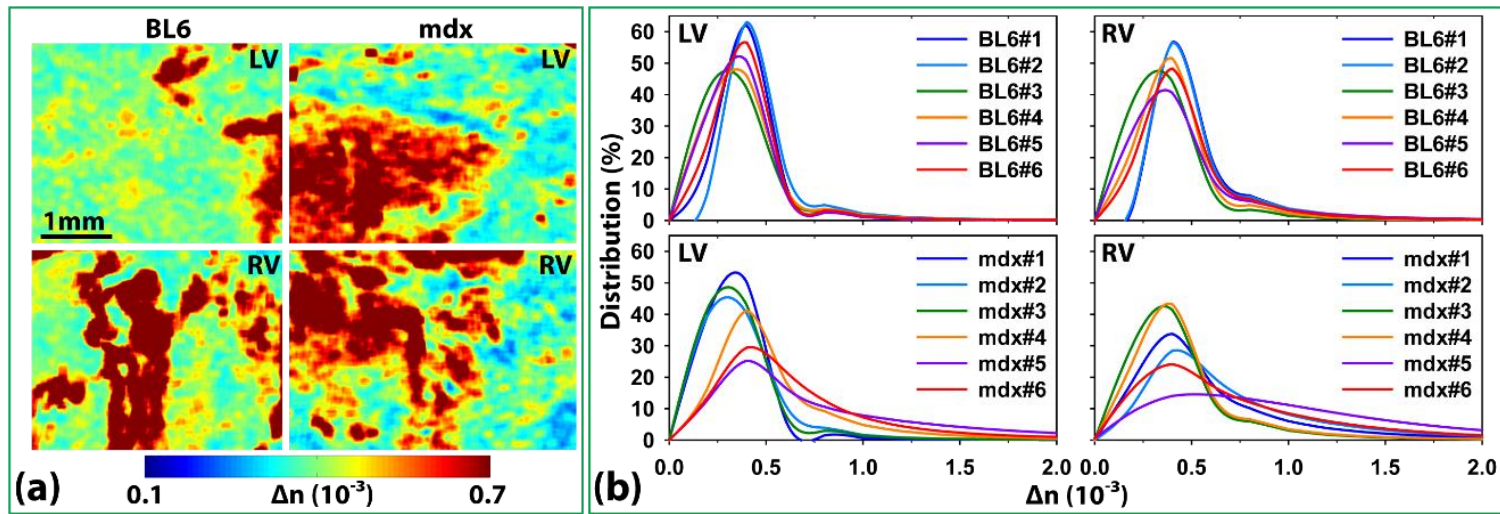

Figure 5-1. Birefringence images and distribution. (a) Example 2D birefringence images at $300 \mu \mathrm{m}$ depth from a BL6 heart and an mdx heart. (b) Distribution of birefringence among six BL6 hearts and six mdx hearts.
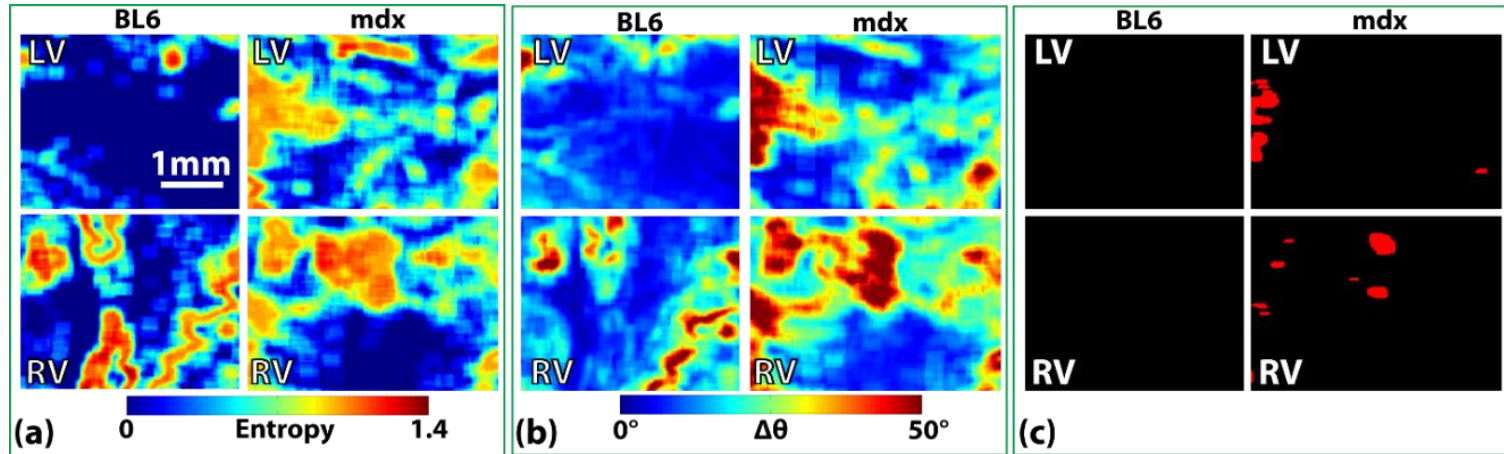

Figure 5-2. 2D images of (a) entropy, (b) circular standard deviation $\Delta \theta$, and (c) segmented tissue damage at $300 \mu \mathrm{m}$ depth from the same hearts shown in Fig. 5-1. Segmentation thresholds: $\mathrm{E}=0.80$ and $\Delta \theta=45.0^{\circ}$.

Both entropy (E) and circular standard deviations of local optic axis $(\Delta \theta)$ were used to quantify the myocardial fiber disarray. 2D maps of entropy and $\Delta \theta$ at a depth of $300 \mu \mathrm{m}$ in LV and RV were illustrated in Figs. 5-2(a) and 5-2(b), respectively. Identified tissue 
damages (marked as red) in the same heart were shown in Fig. 5-2(c). Clearly, the mdx heart had more identified tissue damage than the BL6 heart in both LV and RV.
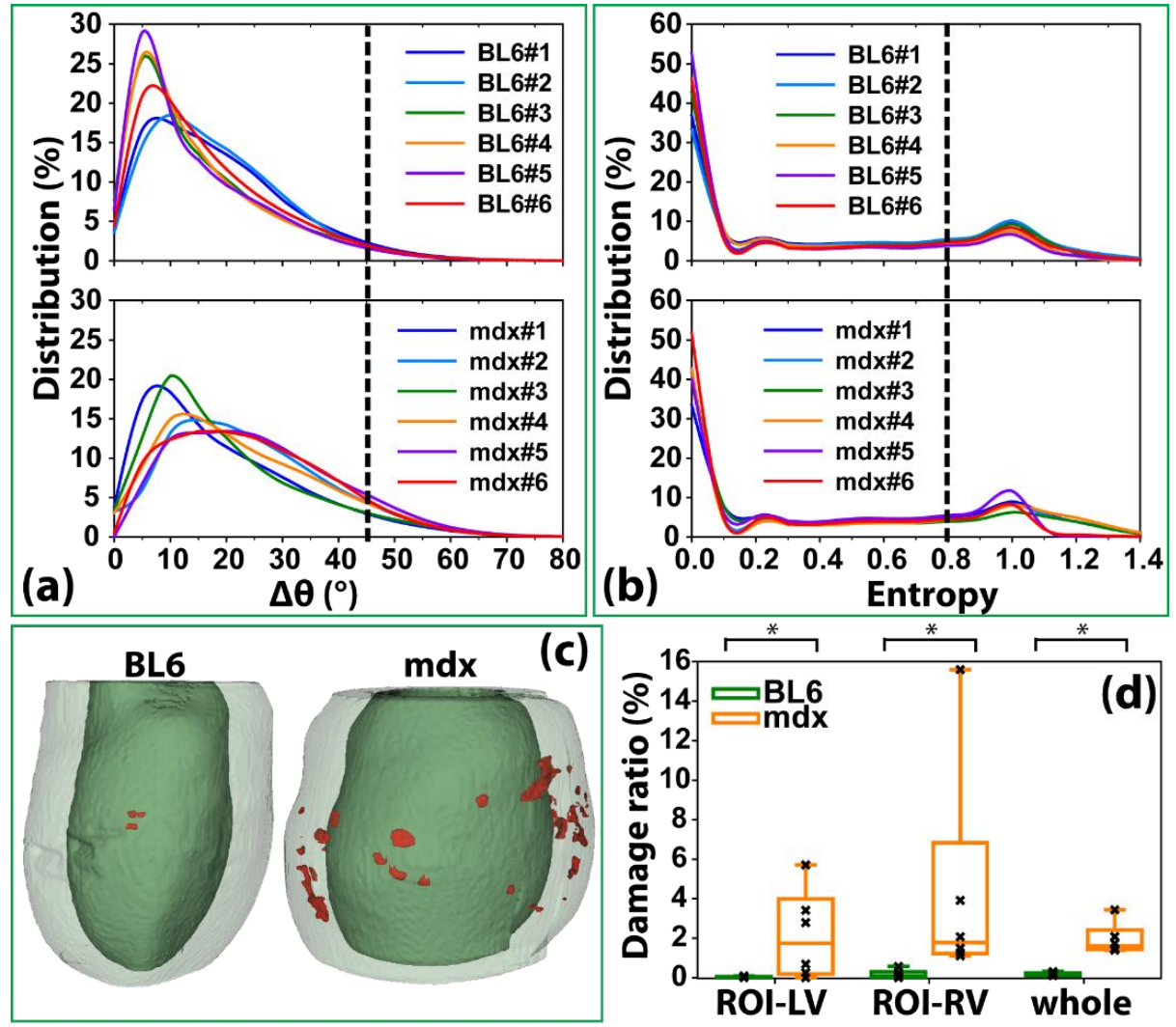

Figure 5-3. Distributions of circular standard deviation $\Delta \theta$ (a) and entropy (b) computed in the whole heart from the surface to $0.5 \mathrm{~mm}$ in depth. (c) 3D visualization of identified muscle damage in the same BL6 heart and mdx heart shown in Fig. 5-1. (d) The box-plot comparison of the damage ratio obtained in six BL6 hearts and six mdx hearts. *Kolmogorov-Smirnov test, $\mathrm{p}=0.0122,0.0013$, and 0.0013 in LV, RV, and whole heart, respectively.

The distributions of $\Delta \theta$ and entropy in the whole heart were shown in Fig. 5-3(a) and 5-3(b), respectively. These distributions were computed from the epicardium until 0.5 $\mathrm{mm}$ in depth. The distributions of $\Delta \theta$ in the $\mathrm{mdx}$ heart showed a less "skewed" profile than the BL6 hearts, which may be caused by potential tissue damage in the mdx hearts. The 
entropy distributions were similar between the two groups.

Figure 5-3(c) shows the segmented muscle damage in the same BL6 heart and mdx heart, and the front views were placed at RVs. Segmentation thresholds of $\Delta \theta$ and entropy were $45.0^{\circ}$ and 0.8 , respectively. Mdx hearts showed significantly more damaged areas than BL6 hearts. Figure 5-3(d) shows the calculated "damage ratio" which was the percentage of the damage volume over the whole volume. The damage ratios in LV and RV were counted within the corresponding evaluation windows. The damage ratio in "whole" was calculated from all the ventricle areas including the interventricular walls. All the results are obtained from the heart surface until $0.5 \mathrm{~mm}$ in depth.

Because the damage ratio was not normally distributed, the two-side KolmogorovSmirnov (K-S) test was used test the group difference between BL6 hearts and mdx hearts. The LV of the mdx hearts showed a greater identified damage volume of $0.2638 \% \pm 1.2680 \%$ than the $0.0026 \% \pm 0.0063 \%$ in BL6 hearts $(\mathrm{p}=0.012)$. The group difference was more significant in RV where the mdx heart had $0.3119 \% \pm 4.1586 \%$ in damage and the BL6 heart damage was $0.0254 \%+0.0580 \%(\mathrm{p}=0.0013)$. Overall, the $\mathrm{mdx}$ hearts showed a greater damage $(\mathrm{p}=0.0013)$ in the whole heart $(0.1975 \%+0.5728 \%)$ than the BL6 hearts $(0.0296 \%+0.0204 \%)$.

To verify identified muscle damage in OPT, histology results were obtained from two pairs of hearts (one BL6 heart and one mdx heart in each pair) with one pair sectioned along the long-axis and the other sectioned along the short-axis. The procedures for histology processing were described in previously (Sec. 5.2.2). HE-stained histology 
images were acquired at $4 \times$ first, and regions with observed muscle damage were imaged again at a higher resolutions (20x). The tissue boundaries in OPT were not similar to that observed from histology due to the morphological changes induced during histology processing. The 3D OPT dataset was initially registered with histology based on the knowledge of the rough sectioning location. Then the 3D OPT data was slightly rotated and tilted while comparing the histology sections with the corresponding OPT 2D projections. The best image registration position was found when locations of tissue damage were best matched between the histology and OPT in all four successive sectioning planes images.
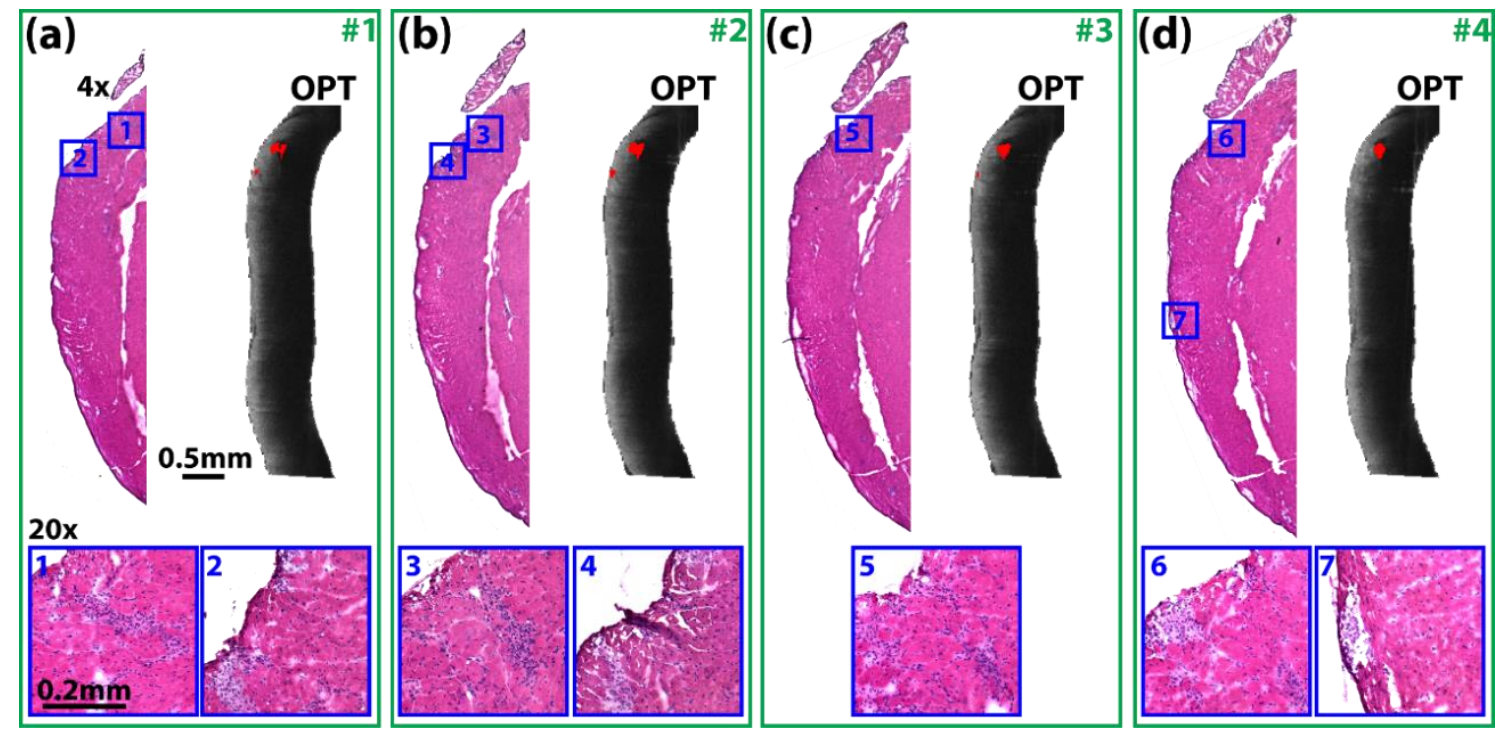

Figure 5-4. A comparison between OPT and histology (HE) sectioned along the long axis of an mdx heart. (a)-(d) Consecutive four histology slices (separated by $80 \mu \mathrm{m}$ ) and the corresponding OPT results of damage (red areas) overlaid on the intensity images. The insets are high resolution (20x) images of the small ROIs marked in boxes. All the $4 \times$ images shared the same scaling bar and all the $20 \times$ images shared the same scaling bar.

Figure 5-4 shows the comparison results of the long-axis sections. The ROI 1, 3, 5, 
and 6 in histology images showed consistent damage over the four consecutive sections. These locations matched with those identified in OPT although the shapes of the damage areas were different. The size of the damage gradually decreased over the distance which was similar in OPT images. The damaged areas showed "scattered" patterns in the histology results, which was not seen in the OPT results due to the evaluation window size used in OPT calculation. In addition, the ROI 2 and 4, also showed similar damage pattern between histology and OPT. However, a surface damage was observed in the $4^{\text {th }}$ long-axis section in histology (region 7), which was not observed in OPT.

Figure 5-5 shows the comparisons between OPT and histology sectioned along the short-axis of an mdx heart. Two regions with consistent muscle damages were observed in histological results: one included ROI 1, 5, 10, and 14and the other included ROI 2, 7, 11, and 15. These two regions were also consistently identified in OPT images. In the first region, the damaged area identified in OPT had about the same location as those identified in histology, but they showed different sizes. The locations of the second damaged area appeared to shift gradually from mid-wall to the endocardium in histology from the first section (\#5) to the last section (\#8). However, their locations were about the same in OPT results. Additional damaged areas (ROI 3, 4, 8, 9, 12, and 16) also showed matched locations between histology and OPT. The ROI 6 and 13 were identified as damaged in histology were not observed in OPT. In addition, OPT showed a damage area at the upper left part of the section \#8, which was not observed in histology. 

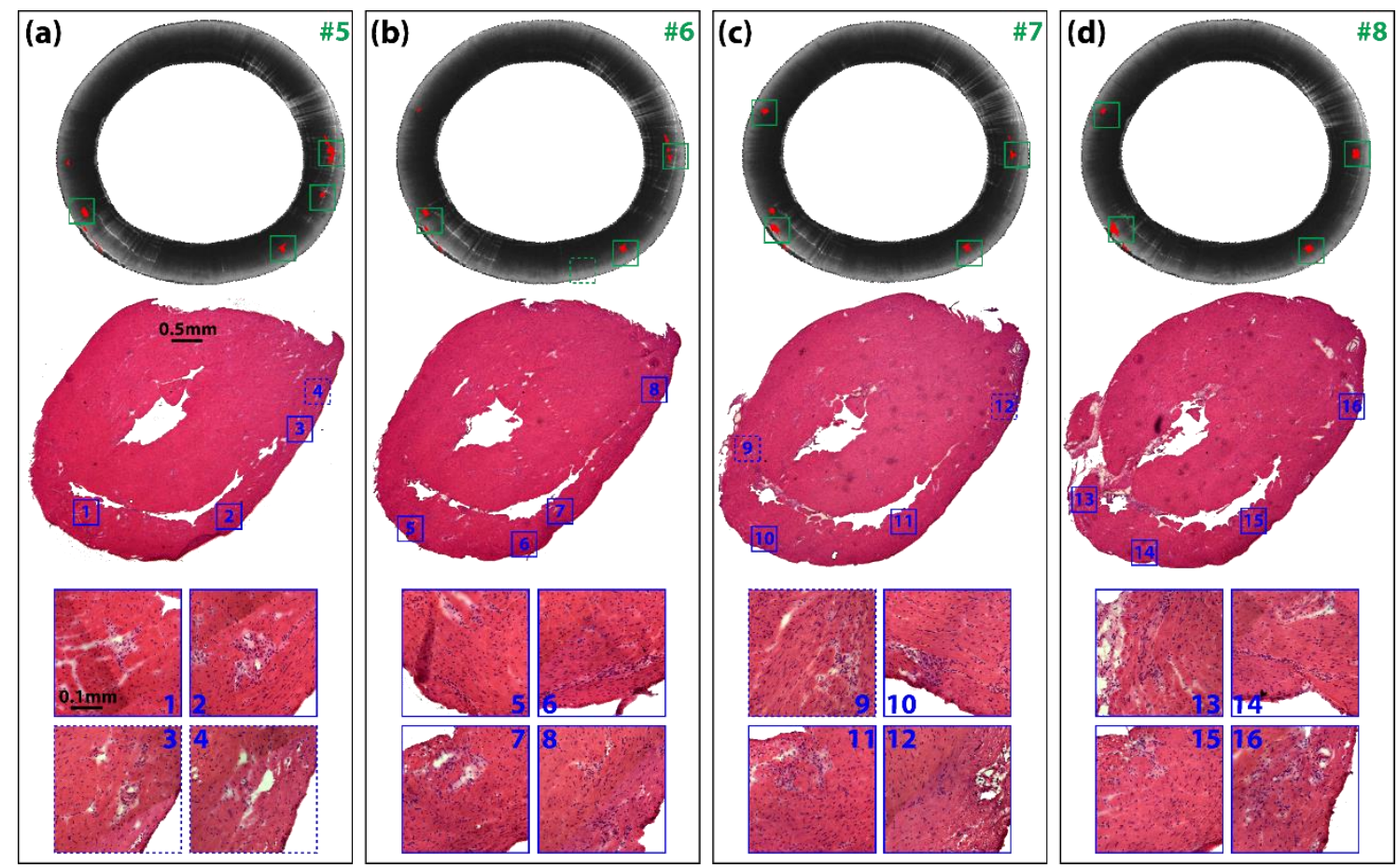

Figure 5-5. A comparison between OPT and histology (HE) sectioned along the short axis of an mdx heart. (a-d) Consecutive four histology slices ( $80 \mu \mathrm{m}$ step) and the corresponding OPT results with segmented damage (red areas) overlaid on the intensity images. The insets are high resolution $(20 \times)$ images of the small ROIs marked in boxes. All the $4 \times$ images shared the same scaling bar and all the $20 \times$ images shared the same scaling bar.

\subsection{Discussion \& conclusion}

The dystrophic pathology in RV is highly related to the prognosis in pulmonary arterial hypertension (Ryan \& Archer 2014). The diaphragm degeneration (Barbin et al. 2016), may lead to ventilator failure, one common cause to the death in DMD (De Bruin et al. 1997; Humbertclaude et al. 2012). In this study, the muscle damage identified from OPT at RVs in the mdx mice, which supports the proposed RV dystrophic pathology.

Unlike the skeletal muscles where the myofibers were very organized, the 
myocardial fibers in heart have more complicated structures. The major cardiac vessels were mixed in the OPT images. They have different fiber organizations (Azinfar et al. 2015) and may interfere in the calculation of $\Delta \theta$ and entropy. Myocardial fibers near the major blood vessel may also follow different fiber orientations, yielding a higher value on $\Delta \theta$ and entropy due to those sudden fiber changes. In addition, orientation transition exists at interventricular walls (Agger et al. 2016) which would also lead to higher values in these two parameters. All these factors may contribute to the errors in identifying tissue damage in OPT and may misidentify true fiber transitions as damage. Segmentation of the blood vessels from myocardial fibers using intensity or birefringence may help to reduce such errors.

All damaged found in histology and OPT are focal and scattered. Conventional histology may have challenges in finding these small damaged areas unless the whole heart is sectioned and analyzed. It is also difficult to observe those small damages in MRI based DTI images due to their insufficient pixel resolution. OPT provides a practical tool to nondestructively identify microscopic heart tissue damage in the entire heart. The small focal damages identified in RV of 7-month-old mdx mice may be one of the early indicators of dystrophic cardiomyopathy (Meyers \& Townsend 2015). Imaging the mdx heart at different ages may provide valuable information on the development of the dystrophic cardiomyopathy. Nevertheless, this study demonstrated the potentials of using OPT in studying the heart structure remodeling in small animal models of human heart diseases. 


\section{Chapter 6. OPT revealed significant fiber disarray in ex vivo skeletal muscles of $\mathrm{mdx}$ mice $^{\S}$}

\subsection{Introduction}

Duchenne muscular dystrophy (DMD) is the most common and severe muscle disease caused by mutations in the dystrophin gene (Emery \& Muntoni 2003). It affects approximately every one of 5,000 male infants. The absence of the dystrophin gene leads to body-wide muscle degeneration and necrosis. Most patients eventually die from respiratory and/or cardiac failure (Emery \& Muntoni 2003). Animal models have been indispensable in our understanding of DMD and developing therapeutic approaches. The mdx $4 \mathrm{cv}$ mouse is a commonly used DMD animal model (Shin et al. 2011) where dystrophin expression is abolished by a nonsense point mutation in exon 53 .

Imaging techniques are important tools for evaluating muscle damage. Conventional histology is often used to identify cellular level muscle damage. Unfortunately it only reveals a small region of interest and is not practical to provide threedimensional (3D) information. Ultrasonography (Zaidman et al. 2010) and magnetic resonance imaging (MRI) (Dunn \& Zaim-Wadghiri 1999) have been explored to evaluate

\footnotetext{
$\S$ This chapter was from a published article: Wang, Y., Zhang, K., Wasala, N.B., Duan, D. and Yao, G., 2015. Optical polarization tractography revealed significant fiber disarray in skeletal muscles of a mouse model for Duchenne muscular dystrophy. Biomedical optics express, 6(2), pp.347-352.
} 
muscle pathology. However, their resolution is still too limited to resolve myofiber damage at the cellular level. Recently, optical coherence tomography (OCT) has shown great promise for skeletal muscle imaging (Klyen et al. 2011; Lovering et al. 2013; Klyen et al. 2014). OCT can achieve high-speed real-time imaging at micro scale resolution. Several studies have tested the feasibility of using OCT to study muscle disease in DMD mouse models. Klyen et al. (Klyen et al. 2011) found that damaged mdx muscle showed a reduced OCT intensity and lower image contrast. Lovering et al. (Lovering et al. 2013) observed atypical muscle architecture in the tibialis anterior (TA) muscles of mdx mice. Most recently Klyen et al. (Klyen et al. 2014) identified necrotic muscle lesions using the optical attenuation coefficient extracted from depth-resolved OCT signals. The attenuation coefficient was significantly increased in injured regions (Klyen et al. 2014).

As in any fibrous tissues, skeletal muscles show strong optical birefringence because the light experiences different optical refractive indices when propagating along and perpendicular to the muscle fiber ( $\mathrm{Li}$ et al. 2008). Muscle damage disrupts the wellorganized fibrous structure and thus can change the muscle birefringent properties. Tissue polarization properties can be imaged at high resolution using polarization-sensitive optical coherence tomography (PSOCT), an important functional extension of conventional OCT. Pasquesi et al. (Pasquesi et al. 2006) were the first to explore the feasibility of using PSOCT to image mdx muscle. They found that birefringence was significantly reduced in exercised mdx muscle. Yang et al. (Yang et al. 2013) recently constructed a novel "parametric image of birefringence" by calculating the slope of the phase retardation measured from $50 \mu \mathrm{m}$ to 
$500 \mu \mathrm{m}$ depth. Such calculated birefringence parameter was significantly smaller in necrotic regions (Yang et al. 2013).

Tissue birefringence is characterized by two parameters: retardance and optic axis. The retardance represents the difference in optical refractive index parallel and perpendicular to the muscle; whereas the optic axis represents the muscle fiber orientation. Although PSOCT has shown great promise for quantifying muscle lesions in the mdx mice model (Pasquesi et al. 2006; Yang et al. 2013), the value of the optic axis has not been explored. Because muscle necrosis disrupts the normal fiber structure, the optic axis would be altered accordingly. In addition, conventional PSOCT only images the "cumulative" polarization properties from the sample surface to a specific imaging depth, which makes it difficult to evaluate "local" lesions at a particular depth (Fan \& Yao 2012c, b). The recently reported "parametric" birefringence imaging (Yang et al. 2013) only resulted in a 2D image instead of a true 3D polarization image. Therefore, new imaging strategies are needed to fully explore the potential of polarization imaging of $3 \mathrm{D}$ lesions in skeletal muscle.

Optical polarization tractography (OPT) was recently developed for visualizing fiber orientation in tissue. It is based on Jones matrix implementation of PSOCT (Fan \& Yao 2013; Wang \& Yao 2013; Wang et al. 2014) and uses advanced Jones calculus based algorithms (Fan \& Yao 2013) to reconstruct the images of depth-resolved "local" optic axis, retardance, and diattenuation. The local optic axis information can be used to construct tissue tractography as in diffusion tensor MRI (Ponrartana et al. 2014) but with 
cellular level resolution (Wang et al. 2014). OPT has recently been applied for imaging cardiac myofiber architecture in the mouse heart (Fan \& Yao 2013; Wang \& Yao 2013; Wang et al. 2014). In this paper, we demonstrated that OPT can effectively identify 3D skeletal muscle damage in freshly excised skeletal muscle samples of mdx $4 \mathrm{cv}$ mice.

\subsection{Method}

\subsubsection{Tissue processing}

Four 7-m-old mdx4cv mice were studied in this study and four age-matched C57BL/6 mice served as typical controls. The tibialis anterior (TA) muscle was excised from each mouse immediately after euthanasia. The muscle samples were rinsed using saline and then imaged using our OPT system. After OPT imaging, the samples were fixed in $4 \%$ paraformaldehyde for at least 2 days. Then tissues were paraffin embedded and sectioned into slices of $5 \mu \mathrm{m}$ thick. The histology slices were stained with hematoxylin and eosin $(\mathrm{H}$ \& E) and histology images were acquired using a Nikon Eclipse E800 microscope equipped with a QImaging RETIGA 1300 camera.

\subsubsection{OPT imaging}

OPT images were acquired using a single-camera spectral domain Jones matrix based PSOCT system. This imaging system has been described in details elsewhere (Fan \& Yao 2012a). As a brief summary, it is a bulk-optical OCT system using an $847.8 \mu \mathrm{m}$ wavelength $(\Delta \lambda=58.3 \mathrm{~nm})$ superluminescent diode (SLD) as the light source. At the sample arm, a $5 \times$, 
f36 mm telecentric scan lens (LSM03-BB, Thorlabs, Newton, NJ) was used as the imaging objective. The light intensity at the sample surface is $5.0 \mathrm{~mW}$. The $2 \mathrm{D}$ raster scan was implemented using a 2D galvanometer scanner and covered an imaging area of $8 \times 8 \mathrm{~mm}^{2}$ on the sample surface. At the detection arm, the interference spectra were acquired using a custom spectrometer equipped with a 1024-pixel line scan camera (AVIIVA SM2, e2v, Milpitas, CA). Images were acquired at a speed of 50k A-lines/sec. This imaging system had a measured lateral resolution of $12.4 \mu \mathrm{m}$ and a measured axial resolution of $<5.9 \mu \mathrm{m}$ in tissue within a $1.5 \mathrm{~mm}$ imaging depth.

The polarization state of the incident light was modulated between right- and leftcircular polarizations using an electric optical modulator. At each incident polarization, the two orthogonal horizontally and vertically polarized backscattered signal were measured separately using two orthogonal reference beams separated in space. From these four signals, the Jones matrix at each pixel was then constructed (Fan \& Yao 2012a, 2013). The entire 3D image dataset had a total of $280 \times 2000 \times 1000$ pixels along the A-, B- and C-scan directions and covered an imaging area of $1.1 \times 8.0 \times 8.0 \mathrm{~mm}^{3}(\mathrm{~A} \times \mathrm{B} \times \mathrm{C})$.

The local depth-resolved polarization properties including (slow) optic axis, retardance and diattenuation were computed from the 3D dataset of Jones matrix at each image pixel. The retardance is defined as $\delta=2 \pi \mathrm{d} \Delta \mathrm{n} / \lambda$ where $\Delta \mathrm{n}$ is the difference in refractive indices along the orthogonal fast and slow optical axes and $\mathrm{d}$ is the physical pathlength of the light. The retardance shown in our results was normalized against the pixel size and had a unit of $\mathrm{rad} / \mathrm{mm}$. The optic axis $\theta$ in our results was the "slow" optic 
axis representing the muscle fiber orientation in the en face (B-C) plane (Fan \& Yao 2013). The diattenuation is defined as the different optical attenuation coefficient along the fast and slow optical axes. The local retardance and diattenuation were first calculated using similar matrix transformation (Fan \& Yao 2013). Then a Jones calculus based iteration algorithm was applied to extract depth-resolved optic axis (Fan \& Yao 2012c, b, 2013).

The final 3D datasets of local optical properties were resized using cubic spline interpolation to produce the same pixel size of $3.9 \mu \mathrm{m}$ in A-, B-, and C-scan. The 3D image data was filtered using a $3 \times 3 \times 3$ (pixel) median filter to improve the signal-to-noise ratio. When visualizing and analyzing the en face images, a $5 \times 5$ median filter was applied to further reduce noise. All the imaging process were implemented in MATLAB. The tractograph was constructed using the local optic axis and visualized using the 3DSlicer software as described in detail previously (Wang \& Yao 2013; Wang et al. 2014).

\subsection{Results and discussions}

Figure 6-1 shows representative cross-sectional and en face images of the freshly excised mdx $4 \mathrm{cv}$ TA muscle. The upper inset of Fig. 6-1d shows the overall cross-sectional histology image (obtained using a $2 \times$ objective) with the corresponding OCT imaging area marked in blue dots. A small necrotic region located at the upper-right part of the muscle sample was marked with red dashed lines. The lower inset of Fig. 6-1d shows the high

resolution images (obtained using a $40 \times$ objective) of the two small region-of-interest 
(ROI) marked in the $2 \times$ histology image. ROI \#1 showed a region of non-damaged muscle; whereas ROI \#2 showed a region of significant muscle necrosis and inflammation.
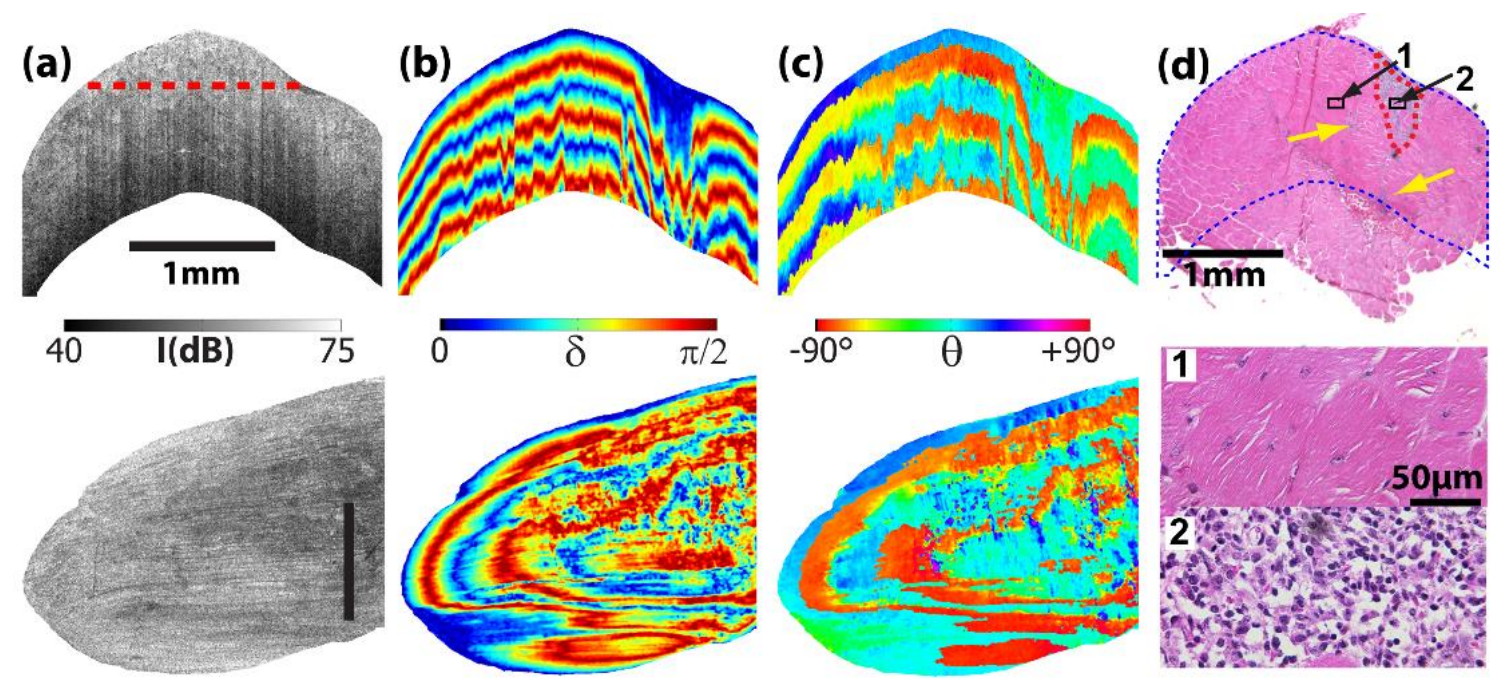

Figure 6-1. Representative images of (a) OCT intensity, conventional cumulative (b) retardance and (c) optic axis of the mdx 4cv TA muscle. The upper row shows crosssectional images and the lower row shows the en face images extracted at the depth marked in the red dashed line in (a). The size bars in (a) indicate $1 \mathrm{~mm}$. (d) The corresponding histology image $(2 \times)$ where the blue dots mark the OCT imaging region. The red dashed line circle indicates the main necrotic region studied here. Yellow arrows indicate additional small necrotic regions observed in the HE histology image. Also shown are high- resolution histology images $(40 \times)$ of the two small region-of-interest as marked in the $2 \times$ histology image.

The muscle damage observed in histology (Fig. 6-1d) was barely detected in the OCT intensity image (Fig. 6-1a) except for a small low-intensity region very close to the surface in the cross-sectional image. However, the cross-sectional images of both cumulative retardance (Fig. 6-1b) and optic axis (Fig. 6-1c) showed altered patterns in areas corresponding to the muscle damage. The banded appearance in these two images is caused by phase wrapping. It appears that the period of the banded pattern was longer in the 
damaged area than in the non-damaged areas. This indicated a smaller retardance value due to muscle damage as described in previous studies (Pasquesi et al. 2006; Yang et al. 2013). It's important to note that the optic axis images in both the cross-sectional and en face planes are difficult to interpret because the cumulative optic axis in conventional PSOCT does not represent the true local fiber orientation (Fan \& Yao 2012b). In addition, the "banded" appearance in conventional retardance and optic axis images made it difficult to identify any small damaged areas (as indicated with yellow arrows in Fig. 6-1d) or the exact boundary of the muscle lesion.
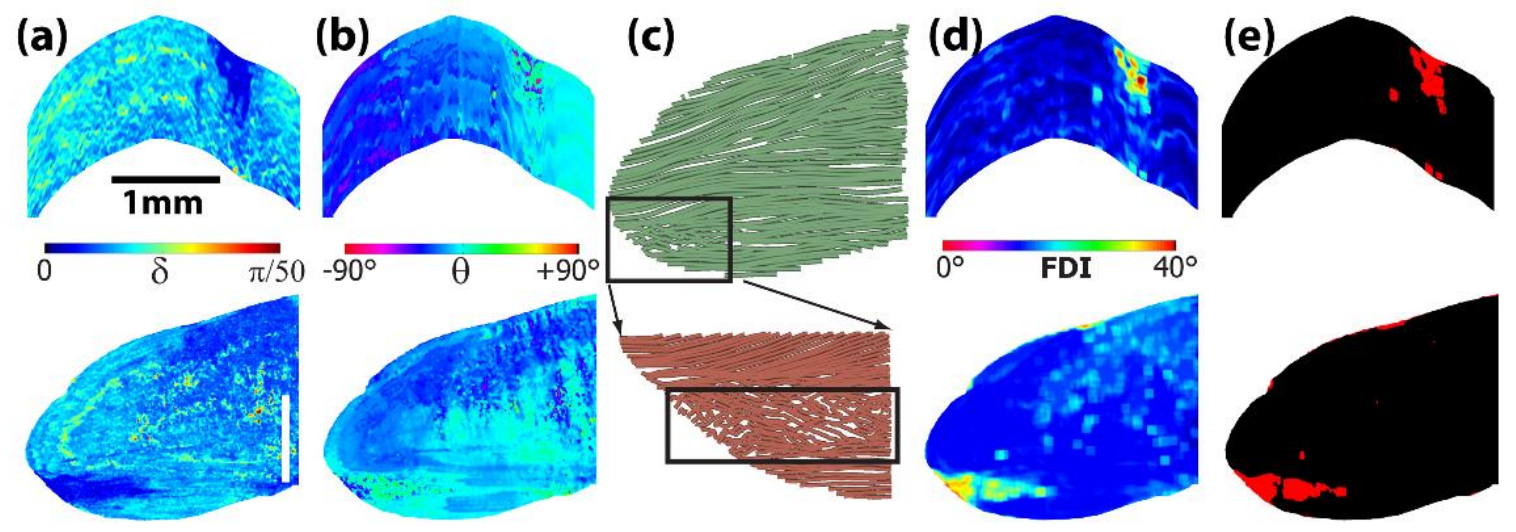

Figure 6-2. The corresponding images of local (a) retardance and (b) optic axis at the same imaging locations as in Fig. 6-1. The size bars in (a) indicate $1 \mathrm{~mm}$. (c) OPT fiber tractography obtained in the same en face plane as in Fig. 6-1. (d) The image of fiber disarray index. (e) Image segmentation using the disarray index (threshold $=16^{\circ}$ ). Yellow arrows indicate small necrotic regions corresponding to those marked in Fig. 6-1(d).

Figure 6-2 shows the OPT results including local retardance (Fig. 6-2a) and local axis (Fig. 6-2b) images of the same sample position as in Fig. 6-1. The necrotic muscle area (Fig. 6-1d) clearly had low local retardance values in both the cross-sectional and en 
face images (Fig. 6-2a). The average retardance was $2.2 \pm 0.4 \mathrm{rad} / \mathrm{mm}$ in the damaged area whereas it was $5.0 \pm 2.0 \mathrm{rad} / \mathrm{mm}$ in the surrounding healthy regions. The images of local optic axis seemed to be relatively homogeneous. However a closer examination indicated that the axis images appeared to be much more noise in regions of muscle damage (Fig. 62b). This feature became more obvious in the fiber tractographic images (Fig. 6-2c). In regions with normal muscle, the fiber bundles were long and well organized. However the fiber orientation varied significantly in damaged area (Fig. 6-2c).

To quantify such variations in optic axis, we defined the "fiber disarray index" FDI by calculating the standard deviation of the local axis within a small $3 \mathrm{D}$ evaluation window:

$$
\mathbf{F D I}=\sqrt{\sum_{i, j, k=1}^{N}\left(\theta_{i, j, k}-\overline{\theta_{i, j, k}}\right)^{2} / N^{3}},
$$

where $\theta \mathrm{i}, \mathrm{j}, \mathrm{k}$ is the local axis value at the pixel $(\mathrm{i}, \mathrm{j}, \mathrm{k})$ and $\overline{\theta_{\mathrm{i}, \mathrm{jk}} \mathrm{k}}$ is the average fiber orientation within the evaluation window. The fiber disarray image can be constructed using the FDI values calculated for each pixel while "sliding" the evaluation windows over the entire image volume. Figure $6-2 \mathrm{~d}$ shows the image of the disarray index calculated using a window size of $\mathrm{N}=9$ pixels (equivalent to $35 \times 35 \times 35 \mu \mathrm{m}^{3}$ ). Figure $6-2 \mathrm{e}$ shows the segmentation result based on the fiber disarray index using a threshold of $16^{\circ}$. The segmentation result showed an excellent agreement with the histology results (Fig. 6-1d).

Figure 6-3 shows the 3D tractography of the TA muscle used in Figs. 6-1 and 6-2 (Fig. 6-3a) and another TA muscle from a different mdx4cv mouse (Fig. 6-3b). For the 
convenience of visualization, the damaged muscles were displayed in a different color from the non-damaged muscle. The damaged muscles showed substantial variations in fiber orientation and a fragmented appearance (Figs. 6-3a and 6-3b). Using a FDI threshold of $16^{\circ}$, the entire damaged muscle volume can be identified in the whole image volume (Figs. 6-3c and 6-3d). In addition to the FDI based segmentation, muscle damage can also be segmented based on local retardance using a threshold of $2.8 \mathrm{rad} / \mathrm{mm}$ (Figs. 6-3e and 6-3f). The two segmentation results appeared similar. Using the ratio of pixel numbers in the damaged and non-damaged muscle regions, the percentage of muscle damage can be calculated. The FDI based segmentation indicated that $4.7 \%$ muscle was damaged in one mouse (Fig. 6-3a); whereas 19.2\% was damaged in the other (Fig. 6-3b). The local retardance based segmentation gave very similar numbers of $4.2 \%$ and $19.4 \%$ in the mice shown in Figs. 6-3a and 6-3b, respectively.

Figures 6-3g and 6-3h show a comparison of the group-averaged fiber disarray index and local retardance obtained in normal C57BL/6 mice, the damaged and nondamaged regions of $m d x 4 c v$ mice. One-way ANOVA with Bonferroni's post-hoc test confirmed that the fiber disarray index was significantly higher $(\mathrm{p}<0.0001)$ in damaged mdx $4 \mathrm{cv}$ muscles $\left(32.8^{\circ} \pm 2.0^{\circ}\right)$ than in non-damaged mdx $4 \mathrm{cv}$ muscles $\left(7.8^{\circ} \pm 1.3^{\circ}\right)$. Similarly, damaged $\mathrm{mdx} 4 \mathrm{cv}$ muscles had a significantly smaller $(\mathrm{p}<0.0001)$ retardance value $(2.3 \pm 0.1 \mathrm{rad} / \mathrm{mm})$ than non-damaged $\mathrm{mdx} 4 \mathrm{cv}$ muscles $(5.4 \pm 0.4 \mathrm{rad} / \mathrm{mm})$. Both parameters appeared to have higher variations in non-damaged $\mathrm{mdx} 4 \mathrm{cv}$ muscles than in muscles from normal C57BL/6 mice. However, no significant difference $(\mathrm{p}>0.05)$ was 
found in either the fiber disarray index or local retardance between non-damaged mdx $4 \mathrm{cv}$ muscles and muscles from normal C57BL/6 mice.
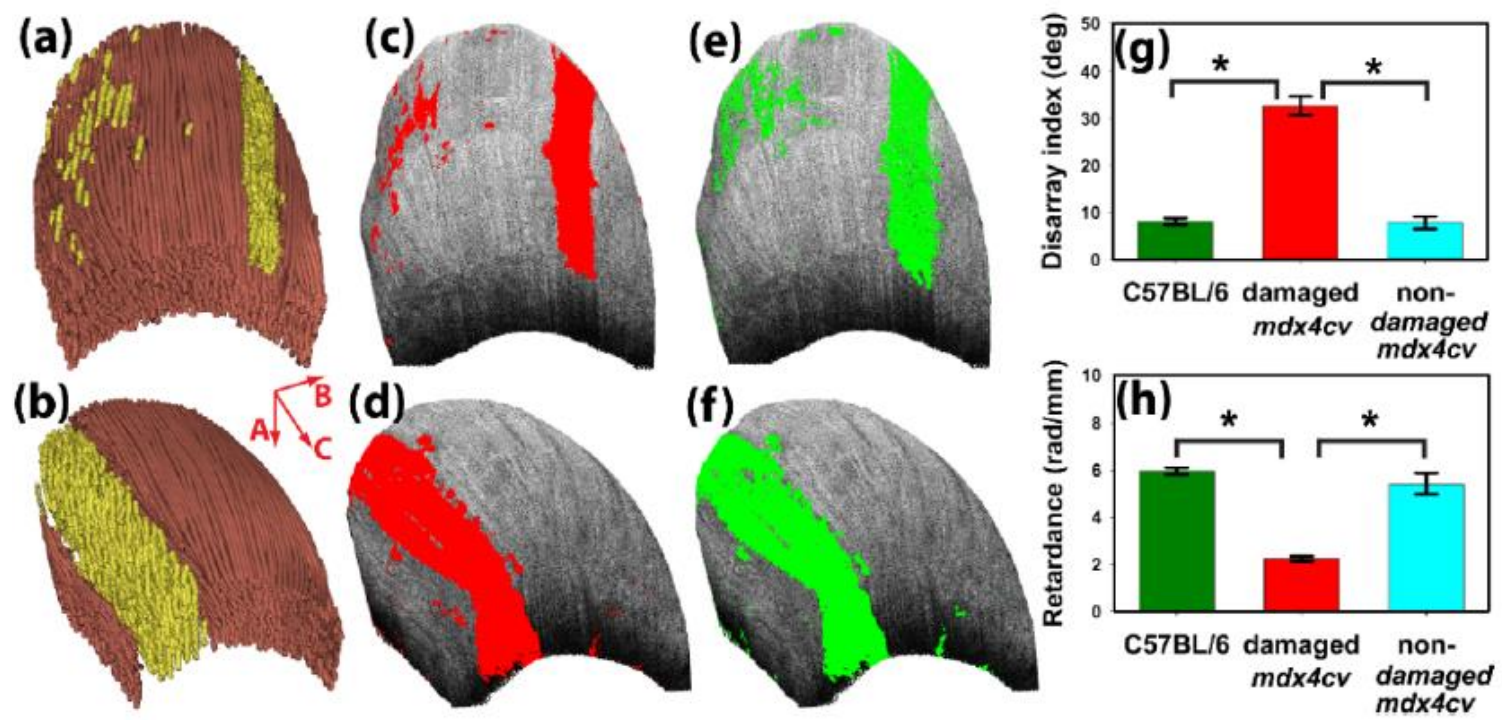

Figure 6-3. (a, b) The 3D OPT images of two TA muscles from two different mdx4cv mice. The mouse used in (a), (c), and (e) was the same as shown in Fig. 6-1 and 6-2. The corresponding segmented results of muscle damage using fiber disarray index with a $16^{\circ}$ threshold are shown in (b) and (d). (e) and (f) show the segmentation results based on local retardance using a threshold of $2.8 \mathrm{rad} / \mathrm{mm}$. Also shown are the statistical comparisons (*, $\mathrm{p}<0.0001)$ of the average $(\mathrm{g})$ fiber disarray index and $(\mathrm{h})$ local retardance obtained in four $\mathrm{mdx} 4 \mathrm{cv}$ mice and four C57BL/6 mice. Error bars represent standard deviations.

\subsection{Conclusion}

Optical polarization tractography (OPT) provides a unique tool for high resolution visualization of tissue fiber organization. In this study, we showed that OPT can successfully image muscle damages in freshly dissected TA muscle of the mdx4cv mice. OPT revealed significant fiber disorganization in regions corresponding to muscle necrosis as confirmed with histology. The local retardance extracted in OPT was significantly lower 
in damaged muscle regions. Both fiber disarray index (FDI) and local retardance can be used to segment the 3D necrotic regions and assess the overall muscle damage. Despite its high speed and superior resolution, as in any OCT based imaging technologies, OPT has a limited imaging depth of $1 \sim 2 \mathrm{~mm}$ in most scattering tissues. However, this limitation can be overcome in practice by using a fine needle probe for deep tissue imaging (Yang et al. 2014). The results shown here demonstrated the great potential of OPT as a valuable imaging tool in muscle research. 


\section{Chapter 7. Dual-angle OPT imaging of absolute 3D fiber orientation in tissue ${ }^{* *}$}

\subsection{Introduction}

Fibrous tissue exists in many parts of the body, for example in skeletal muscle, heart, and brain. A well-organized fiber architecture is essential for maintaining normal physiological functions in these tissues. As an example, the unique helical myofiber organization in the heart is critical for regulation of blood pumping (Taccardi et al. 1994). A technology that can image fibrous organization with sufficient resolution and field-of-view would be valuable for our understanding and assessment of the important structure-function relationship in these tissues (Van Donkelaar et al. 1999; Buckberg et al. 2008). Traditional histology sectioning (Streeter Jr et al. 1969) can achieve cellular level resolution, but is labor-intensive and only practical for sampling a small area in fixed tissues. Diffusiontensor based magnetic resonance imaging (DTI) (Basser et al. 1994) has been established for imaging global 3D fiber organization, especially in brain and heart. However the spatial resolution of current DTI is limited to sub-millimeters which is insufficient for imaging thin tissues or small structural changes.

\footnotetext{
** This chapter was from a published article: Wang Y., Ravanfar M., Zhang K., Duan D., and Yao G., 2016. Mapping 3D fiber orientation in tissue using dual-angle optical polarization tractography. Biomedical Optics Express, 7(10), pp. 3855-3870.
} 
High resolution optical coherence tomography (OCT) has been investigated to study fiber orientation in tissues (Fleming et al. 2008; Ambrosi et al. 2012; Goergen et al. 2012; Gan \& Fleming 2013; Wang et al. 2015a). However, fiber detection based on intensity contrast can be affected by non-fiber related intensity variations and only works in tissues with large fibers. We recently developed optical polarization tractography (OPT) for imaging tissue fiber orientation (Fan \& Yao 2012c, b, 2013). OPT is based on Jones matrix polarization-sensitive OCT (PSOCT) and uses the depth-resolved "local" optic axis to construct the 3D fiber tractographic images of the sample. OPT has been recently applied to imaging fiber structure in heart muscle (Wang \& Yao 2013), skeletal muscle (Wang et al. 2015b), and blood vessels (Azinfar et al. 2015). Histology validation showed that OPT can image detailed fiber architectures in tissue with histology-like resolution (Wang et al. 2014).

The fiber orientation measured in OPT represents the "projected" orientation in an imaging plane perpendicular to the incident light (Wang et al. 2014). This projection angle in a $2 \mathrm{D}$ plane cannot fully represent the true fiber orientation in the $3 \mathrm{D}$ space, especially when a fiber is inclined toward the incident light. We show in this study that images of true 3D fiber orientation can be constructed by applying OPT to image a sample at two different angles. Using more than one incident angle to resolve the absolute fiber orientation in polarization imaging has been explored before in a few studies (Ugryumova et al. 2006; Wallenburg et al. 2010; Kasaragod et al. 2012; Lu et al. 2014; Liu et al. 2016). For example, a "dual-projection" polarimetry imaging system was developed to obtain 
orientation map in tissue slice (Wallenburg et al. 2010). Analyzing cumulative PSOCT results obtained at different incident angles was also used to derive the representative 3D fiber orientation in cartilage (Ugryumova et al. 2006; Kasaragod et al. 2012; Lu et al. 2014) and muscle (Liu et al. 2016). Unfortunately, these earlier studies did not reconstruct the depth-resolved 3D fiber orientation in the sample. In sharp contrast, here we show that dual-angle OPT successfully reveals the full high-resolution 3D images of 3D fiber orientation in complicated biological tissues such as the articular cartilage.

\subsection{Methods}

\subsubsection{OPT algorithm and imaging system}

The OPT method uses Jones calculus based algorithms to calculate the "local" optic axis from cumulative measurements in Jones-matrix PSOCT as described in detail previously (Fan \& Yao 2013; Wang \& Yao 2013). Briefly, the "round-trip" pixel-wise Jones matrix of the entire imaging volume $\mathbf{J}_{\mathrm{RT}}\left(\phi_{\mathrm{n}}, 2 \rho_{\mathrm{n}}\right)$ was constructed, where the cumulative complex retardance $\rho_{\mathrm{n}}$ and axis $\phi_{\mathrm{n}}$ from the $\mathrm{n}^{\text {th }}$ depth layer can be calculated. With $\rho_{\mathrm{n}}$ and $\phi_{\mathrm{n}}$, a new Jones matrix $\mathbf{J}_{\mathrm{L}}\left(\phi_{\mathrm{n}}, \rho_{\mathrm{n}}\right)$ was constructed and another complex matrix $\mathbf{K}(\mathrm{n}+1)$ was generated as:

$$
\mathbf{K}(n+1)=\mathbf{J}_{\mathrm{L}}^{-1}\left(\phi_{n}, \rho_{n}\right) \mathbf{J}_{\mathrm{RT}}(n+1) \mathbf{J}_{\mathrm{L}}^{-1}\left(\phi_{n}, \rho_{n}\right)
$$

The "local" complex retardance at the $\mathrm{n}^{\text {th }}$ layer $\xi_{\mathrm{L}}(\mathrm{n})=\delta_{\mathrm{L}}+\mathrm{i} \sigma_{\mathrm{L}}$ was obtained using 
the eigenvalues of $\mathbf{K}(n+1)$. The imaginary $\sigma_{L}$ was eliminated from $\mathbf{J}_{R T}(n)$ in subsequent calculations to create a diattenuation-free matrix $\mathbf{J}_{\mathrm{RT}}^{\prime}(\mathrm{n})$. The "local" optic axis $\beta$ was derived using an iterative process from the first layer where the axis was obtained directly from the round-trip Jones matrix $\mathbf{J}_{\mathrm{RT}}^{\prime}(1)$ using eigen-decomposition. The derived "local" axis and retardance from the $1^{\text {st }}$ to the $(\mathrm{n}-1)^{\mathrm{th}}$ layer were used to construct the "single-trip" Jones matrix $\mathbf{J}_{\mathrm{ST}}(\mathrm{n}-1)$. Then the $\mathrm{n}^{\text {th }}$ layer local axis was obtained from the local Jones matrix $\mathbf{J}_{\mathrm{L}}(\mathrm{n})$ :

$$
\mathbf{J}_{\mathrm{L}}^{\mathrm{T}}(n) \mathbf{J}_{\mathrm{L}}(n)=\left[\mathbf{J}_{\mathrm{ST}}^{\mathrm{T}}(n-1)\right]^{-1} \mathbf{J}_{\mathrm{RT}}^{\prime}(n)\left[\mathbf{J}_{\mathrm{ST}}(n-1)\right]^{-1} .
$$

The local optic axis $(\beta)$ was used to construct the tractography using the streamline functions in the Matlab software.

The OPT algorithms are general and can be applied to any Jones matrix PSOCT systems. For the purpose of demonstration, we used a single-camera spectral-domain Jones-matrix PSOCT in this study. The details of this system has been reported elsewhere (Fan \& Yao 2010b, 2012a). It utilized a $0.85 \mu \mathrm{m}$ superluminescent diode (SLD) as the light source and had an axial resolution of $8.2 \mu \mathrm{m}$ in air and a lateral resolution of $12.4 \mu \mathrm{m}$. The system acquired a $6 \times 6 \times 1.1 \mathrm{~mm}^{3}$ sample volume $(2000 \times 1000 \times 280$ pixels in $\mathrm{B} \times \mathrm{C} \times \mathrm{A}$ scans $)$ at a $50 \mathrm{kHz} \mathrm{A}$-scan rate. In this study, the obtained 3D dataset was interpolated in the lateral and axial directions to achieve an isotropic pixel size of $6.0 \mu \mathrm{m}$ in all A-, B-, and C-scan directions.

\subsubsection{Dual-angle OPT}


Figure 7-1 shows the laboratory coordinate system established using the orthogonal A-, B-, and C-scan directions labeled as the z-, $\mathrm{y}_{-}$, and $\mathrm{x}$-axis, respectively. The origin is located at the intersection of the incident light and the sample surface (Fig. 7-1).

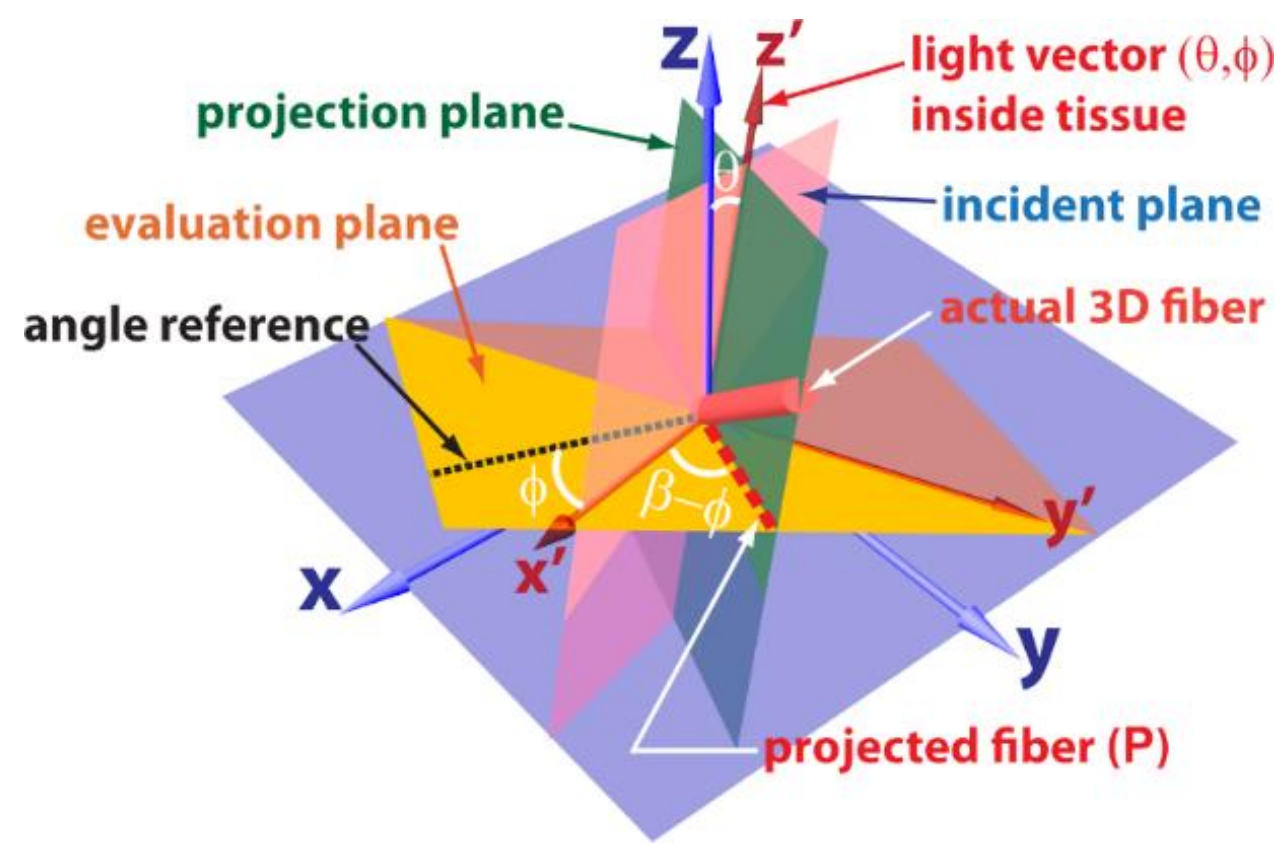

Figure 7-1. An illustration of the OPT measurement geometry. The laboratory coordinate system is formed by the $x-y-z$ vectors. The light is initially incident along the $z$-axis, but is refracted to the $z^{\prime}$-axis inside the sample. OPT measures the fiber orientation $\beta$ within the evaluation plane $\left(x^{\prime}-y^{\prime}\right)$ which is perpendicular to the light direction $\left(z^{\prime}\right)$ inside the sample. The actual fiber lies within the projection plane formed by the light direction and the fiber.

The incident light is refracted at the tissue surface depending on the incident angle and enters the tissue as the $\mathrm{z}^{\prime}$-axis. The local optic axis measured in OPT is equivalent to the orientation of the actual fiber projected at the "evaluation plane" (the $\mathrm{x}^{\prime}-\mathrm{y}$ ' plane in Fig. 7-1) which is determined based on the law of optical refraction in 3D. This evaluation plane is perpendicular to the refracted light ( $\mathrm{z}^{\prime}$-axis) inside the tissue, the incident plane $\left(\mathrm{z}-\mathrm{z}^{\prime}-\mathrm{x}^{\prime}\right)$, and the projection plane (formed by $\mathrm{z}^{\prime}$ and the $3 \mathrm{D}$ fiber). It is important not to confuse the 
"evaluation plane" with the conventional en face plane (x-y plane) in OCT imaging. In addition, OPT measures fiber orientation $(\beta)$ with respect to the horizontal linear polarization in our system, which is aligned with the x-axis in the laboratory coordinates. This reference direction also needs to be projected to the evaluation plane by rotating $\theta$ around the $y^{\prime}$-axis (normal to the incident plane) and is marked as the "angle reference" $\left(0^{\circ}\right)$ in Fig. $7-1$.

The refracted light (the $\mathrm{z}^{\prime}$ axis in Fig. 7-1) can be obtained based on the Snell's law using the incident light vector $\mathbf{I}=\left[I_{\mathrm{x}}, I_{\mathrm{y}}, I_{\mathrm{z}}\right]^{\mathrm{T}}$ and the surface normal vector $\mathbf{N}=\left[N_{\mathrm{x}}, N_{\mathrm{y}}, N_{\mathrm{z}}\right]^{\mathrm{T}}$

$$
\mathbf{L}=\left[L_{x}, L_{y}, L_{z}\right]^{\mathrm{T}}=\mathbf{I} / n+\left(\cos \theta_{i}-\sqrt{n^{2}-\sin ^{2} \theta_{i}}\right) \mathbf{N} / n,
$$

where $\mathrm{n}$ is the tissue refractive index. The $\theta_{i}$ is the incident angle so that $\cos \theta_{i}=-\mathbf{N} \bullet \mathbf{I}$. The refracted light vector can also be represented as $\mathbf{L}=[\sin \theta \cos \phi, \sin \theta \sin \phi, \cos \theta]^{\mathrm{T}}$ where the polar angle $\theta$ and azimuthal angle $\phi$ are defined as $\theta=\cos ^{-1}\left(\left|\mathrm{~L}_{\mathrm{z}}\right|\right), \phi=\tan ^{-1}\left(\mathrm{~L}_{\mathrm{y}} / \mathrm{L}_{\mathrm{x}}\right)$.

The orientation $\beta$ measured in OPT defines a $2 \mathrm{D}$ unit vector which represents the projected fiber inside the evaluation plane $\left(\mathrm{x}^{\prime}-\mathrm{y}^{\prime}\right.$ in Fig. 7-1). This projected vector (Fig. 71) can be expressed in the laboratory coordinate $(x-y-z)$ as:

$$
\mathbf{P}=\left[\begin{array}{l}
\cos \theta \cos \phi \cos (\beta-\phi)-\sin \phi \sin (\beta-\phi) \\
\cos \theta \sin \phi \cos (\beta-\phi)+\cos \phi \sin (\beta-\phi) \\
-\sin \theta \cos (\beta-\phi)
\end{array}\right]
$$

The actual 3D fiber cannot be determined by the $\beta$ measured in a single-scan OPT measurement. However this fiber must be located within the projection plane (Fig. 7-1) 
formed by the refracted light and the projected fiber. If the fiber orientation is measured using a different projection plane, the 3D fiber orientation can be fully determined as the intersection line of the two projection planes. The second measurement can be performed by rotating the sample around a predefined axis. The projection plane can be conveniently identified using its normal vector $\mathbf{M}$ by the cross-product of $\mathbf{L}$ in Eq. (3) and $\mathbf{P}$ in Eq. (4):

$$
\mathbf{M}=\mathbf{P} \times \mathbf{L}=\left[\begin{array}{c}
\sin \phi \cos (\beta-\phi)+\cos \theta \cos \phi \sin (\beta-\phi) \\
-\cos \phi \cos (\beta-\phi)+\cos \theta \sin \phi \sin (\beta-\phi) \\
-\sin \theta \sin (\beta-\phi)
\end{array}\right]
$$

In the second measurement, the refracted light has a different direction $\left(\theta^{\prime}, \phi^{\prime}\right)$ leading to a new projected fiber orientation $\beta^{\prime}$ and projection plane with a normal vector $\mathbf{M}^{\prime}\left(\theta^{\prime}, \phi^{\prime}, \beta^{\prime}\right)$. The 3D fiber orientation vector $\mathbf{F}$ of the sample was calculate as:

$$
\mathbf{F}=\left[f_{x}, f_{y}, f_{z}\right]^{\mathrm{T}}=\mathbf{M}(\theta, \phi, \beta) \times \mathbf{M}^{\prime}\left(\theta^{\prime}, \phi^{\prime}, \beta^{\prime}\right)
$$

To facilitate the evaluation of $3 \mathrm{D}$ orientation, the constructed $3 \mathrm{D}$ fiber vector $\mathbf{F}$ at each pixel can be projected into the three orthogonal planes $y-x(B-C), z-x(A-C)$ and $z-y$ (A-B) to obtain the corresponding 2D projection angles:

$$
\left\{\begin{array}{l}
\theta_{\mathrm{AC}}=\tan ^{-1}\left(f_{\mathrm{z}} / f_{\mathrm{x}}\right)=\tan ^{-1}\left(\cot \theta_{f} / \cos \phi_{f}\right) \\
\theta_{\mathrm{AB}}=\tan ^{-1}\left(f_{\mathrm{z}} / f_{\mathrm{y}}\right)=\tan ^{-1}\left(\cot \theta_{f} / \sin \phi_{f}\right) \\
\theta_{\mathrm{BC}}=\tan ^{-1}\left(f_{y} / f_{x}\right)=\phi_{f}
\end{array}\right.
$$

where $\theta_{\mathrm{f}}$ and $\phi_{\mathrm{f}}$ are the polar and azimuthal angles of the 3D fiber direction. 


\subsubsection{Image registration}

In this study, dual-angle OPT was implemented by imaging the sample placed at two different rotation angles $\left(\gamma_{1}\right.$ and $\left.\gamma_{2}\right)$ around the $\mathrm{x}$-axis (C-scan). The second image (at $\left.\gamma_{2}\right)$ was registered with the first image (at $\left.\gamma_{1}\right)$ to calculate both $\mathbf{M}(\theta, \phi, \beta)$ and $\mathbf{M}^{\prime}\left(\theta^{\prime}, \phi^{\prime}, \beta^{\prime}\right)$ in the laboratory coordinates (Eqs. $5 \&$ 6). For this reason, the second image volume including the $\mathbf{M}^{\prime}$ were rotated back by $\gamma_{1}-\gamma_{2}$ around the x-axis. Due to the optical refraction effect at the tissue surface, the incident light deviated from the initial incident direction (the z-axis) which was defined by the A-scan direction of the imaging system. Such an effect changed the orientation of the "evaluation" plane and distorted the original 3D rectangular image grid $(i, j, k)$ and was corrected before image registration (Wang et al. 2014). The sample surface boundary was first identified using an intensity-based thresholding method as in our previous studies (Fan \& Yao 2013; Wang \& Yao 2013). A 5×5 pixel median filter was applied to remove noisy pixels on the resulting 2D surface. The surface normal vector was then calculated using the "surfnorm" function in Matlab. The light direction $(\theta, \phi)$ inside the sample after refraction was calculated using Eq. (3). The actual pixel coordinates ( $i^{\prime}, j^{\prime}$, $\left.\mathrm{k}^{\prime}\right)$ for the original pixel $(i, j, k)$ were calculated as:

$$
\left\{\begin{array}{l}
i^{\prime}=i+k \sin \theta \cos \phi \\
j^{\prime}=j+k \sin \theta \sin \phi . \\
k^{\prime}=k \cos \theta
\end{array}\right.
$$

A new rectangular image matrix was then constructed using 3D cubic spline interpolation based on the actual pixel coordinates $\left(i^{\prime}, j^{\prime}, k^{\prime}\right)$ obtained in Eq. (8). And the $\mathbf{M}$ 
vector was measured for each pixel using Eq. (5). This refraction correction procedure was applied to both image volumes acquired at two different angles in the dual-angle imaging procedure. Then the corrected image volume obtained at the second imaging angle was rotated back to register with that obtained at the first imaging angle. The 3D fiber orientation was then calculated for each registered pixel using Eq. (6). All image processing was implemented in Matlab. The 3DSlicer software (www.slicer.org) was used for visualizing the $3 \mathrm{D}$ OPT results.

\subsection{Results}

The dual-angle OPT method was first verified by imaging the 3D fiber orientation in the mouse extensor digitorum longus (EDL) muscle that was placed at various known orientations. Once the 3D fiber direction was measured at one sample position, it can be used to predict fiber direction at other sample positions. Therefore, the results obtained in dual-angle OPT can be validated by comparing the expected values with the measured values at various sample positions. The accuracy and capability of dual-angle OPT was further investigated and analyzed by imaging the 3D fiber structure in the mouse tibialis anterior (TA) muscle and the bovine articular cartilage.

\subsubsection{Extensor digitorum longus (EDL) muscle}

Figure 7-2(a) illustrates the geometry of the EDL muscle in the dual-angle OPT test. The muscle sample was excised from a C57BL/6 mouse and fixed in 4\% paraformaldehyde. 
The muscle sample was mounted on a two-axis rotation stage carefully aligned with the scanning directions to provide accurate rotations around the $\mathrm{x}$-axis $(\mathrm{C}$-scan) and $\mathrm{y}$-axis (Bscan). To implement the dual-angle scanning, the EDL muscle was first scanned at $\gamma_{1}=-20^{\circ}$ (rotation around the $\mathrm{x}$-axis); then imaged again at $\gamma_{2}=+20^{\circ}$ after rotating the sample by $40^{\circ}$ around the $\mathrm{x}$-axis.
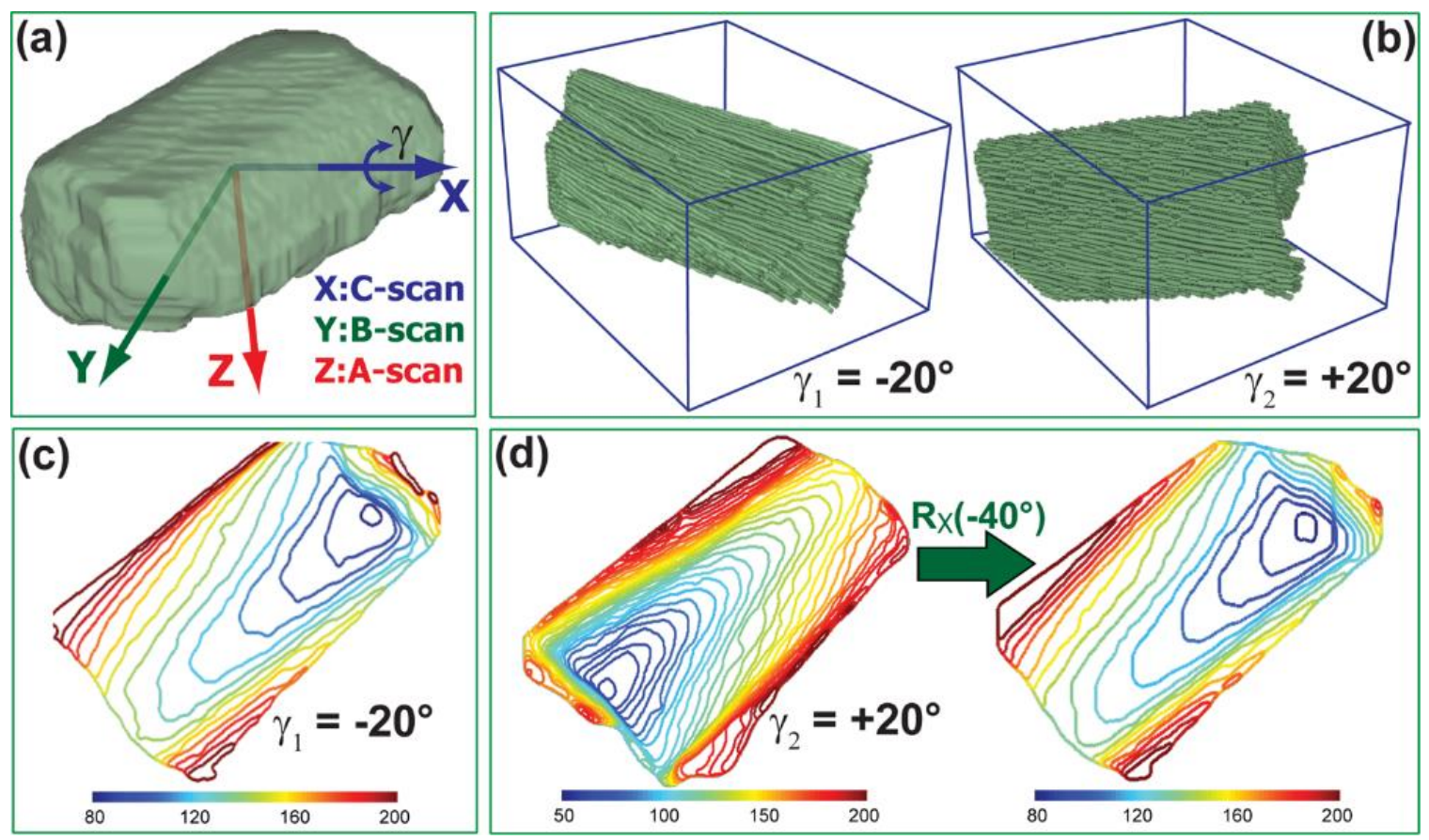

Figure 7-2. Single-scan OPT and registration of two image volumes acquired in dual-angle OPT. (a) A schematic illustration of the geometry of the dual-angle OPT imaging. The EDL sample was rotated in the laboratory coordinates by $\gamma_{1}$ and $\gamma_{2}$ around the $\mathrm{x}$-axis to complete the dual angle imaging. (b) The single-scan OPT of the EDL muscle obtained at $\gamma_{1}=-20^{\circ}$ and $\gamma_{2}=+20^{\circ}$. (c) The surface profile of the EDL muscle imaged at $\gamma_{1}=-20^{\circ}$. (c) The surface profiles of the same EDL muscle imaged at $\gamma_{2}=+20^{\circ}$ and after rotating back by $-40^{\circ}$ around the $\mathrm{x}$-axis to be registered with the results in (c). The unit in the contour plots (c) and (d) represents the height of the sample surface (in "pixels" along the z-axis).

Figure 7-2(b) shows the conventional single-scan OPT of the EDL muscle at two 
different angles $\left(\gamma_{1}\right.$ and $\left.\gamma_{2}\right)$ which were later used for dual-angle reconstruction. The fiber orientation measured in the single-scan OPT was the 2D projected angle of the 3D fiber in the evaluation plane (Fig. 7-1). As described in previous studies (Wang \& Yao 2013; Wang et al. 2015b), such projected tractography was constructed within the equi-transmural layer from the tissue surface.

Before dual-angle image construction, the registration of the two image volumes measured at $\gamma_{1}$ and $\gamma_{2}$ was examined by comparing their surface profiles. As expected, the "contour" profiles of the sample surface obtained at $\gamma_{1}=-20^{\circ}$ (Fig. 7-2(c)) and $\gamma_{2}=+20^{\circ}$ (Fig. 7-2(d)) were noticeably different due to their different image positions. To register the sample surface measured at $\gamma_{2}=+20^{\circ}$ with that obtained at $\gamma_{1}=-20^{\circ}$, the image data acquired at $\gamma_{2}=20^{\circ}$ was rotated back by $40^{\circ}$ by multiplying $\mathbf{R}_{\mathrm{x}}\left(-40^{\circ}\right)$ to the $3 \mathrm{D}$ coordinates of each image pixel, where $\mathbf{R}_{\mathbf{x}}$ is the standard 3D rotational matrix around the $\mathrm{x}$-axis. The resulting surface (Fig. 7-2(d), right) appeared very similar to the one shown in Fig. 7-2(c). Due to the alignment errors of the rotational stages, the optimal rotation angle was slightly adjusted within a small range $\left(<2^{\circ}\right)$ to minimize the pixel-wise error between the two surface profiles. Quantitatively, 95\% of the sample surface had $<5$-pixel difference after registration.

During the validation process, the above dual-angle OPT procedure was performed multiple times while placing the EDL muscle at six different positions from $\alpha=-30^{\circ}$ to $+20^{\circ}$ in a step of $10^{\circ}$ around a "validation" axis $\mathbf{V}$ in the y-z plane (Fig. 7-3(a)). The rotational axis $\mathbf{V}$ had a polar angle $\theta_{v}=90^{\circ}-\gamma_{1}$ with the $\mathrm{z}$-axis. Two example 3D image volumes 
obtained at $\alpha=-30^{\circ}$ and $+20^{\circ}$ were illustrated in Fig. 7-3(a) using different colors. These six positions induced considerable changes of fiber orientation in the three orthogonal projection planes $(x-z, z-y$, and $x-y$ for $\mathrm{AC}, \mathrm{AB}$, and $\mathrm{BC}$, respectively). The coordinates and axis $\mathbf{V}$ shown in Fig. 7-3(a) were actually located inside the tissue during the experiment (see Fig. 7-2(a)).
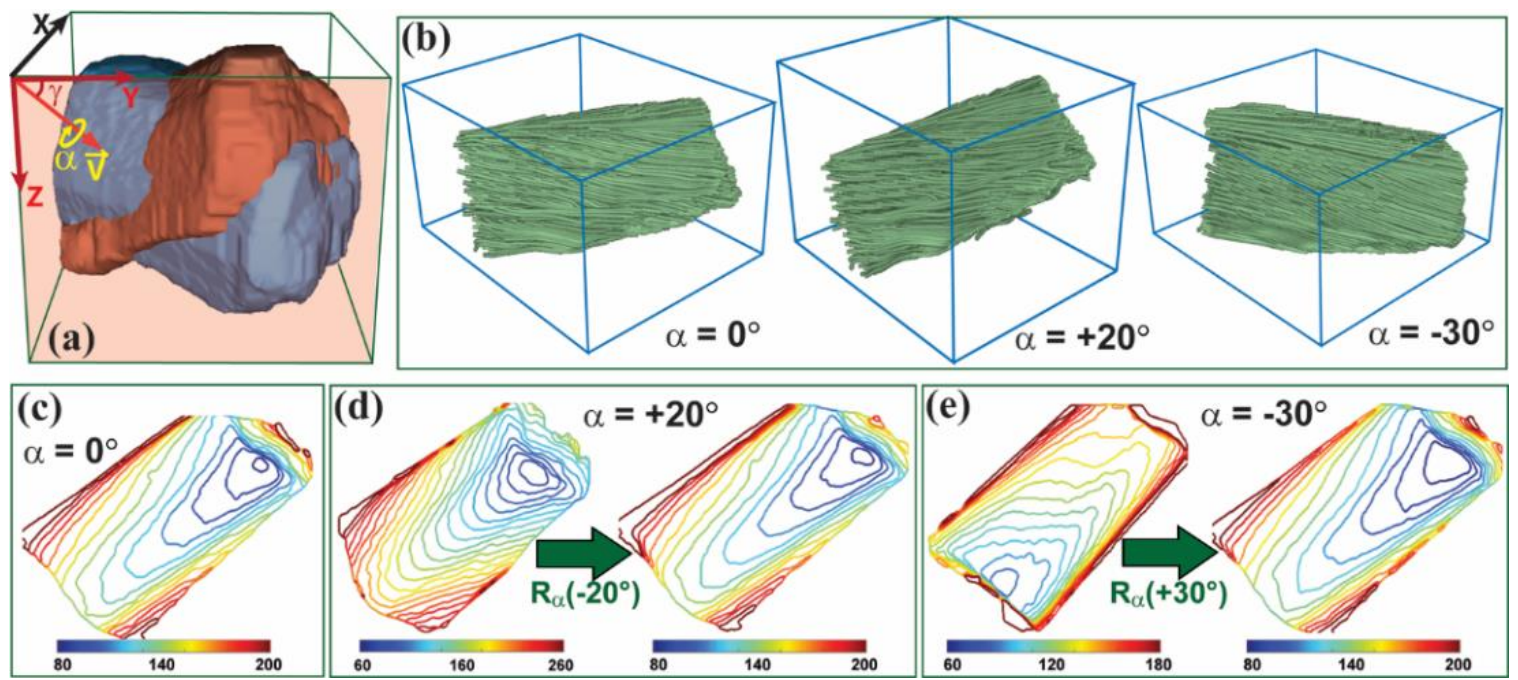

Figure 7-3. 3D dual-angle OPT and registration of multiple image volumes obtained at different 3D positions. (a) A schematic illustration of rotating the EDL muscle from $\alpha=-$ $30^{\circ}$ to $\alpha=+20^{\circ}$ around the validation axis $\mathbf{V}$. (b) The 3D tractography of the EDL muscle placed at $\alpha=0^{\circ}, \alpha=+20^{\circ}$, and $\alpha=-30^{\circ}$ obtained using the dual-angle OPT at each position. (c) The surface profiles of the sample located at $\alpha=0^{\circ}$. The images in (d) and (e) show the corresponding surface profile measured at $\alpha=+20^{\circ}$ and $\alpha=-30^{\circ}$, and after rotating the image volumes back by $-20^{\circ}$ and $+30^{\circ}$ around the validation axis to be registered with (c). The unit in the contour plots (c), (d), and (e) represents the height of the sample surface (in "pixels" along the z-axis).

At each sample position $\alpha$, 3D fiber orientation was constructed using dual-angle OPT obtained at $\gamma_{1}=-20^{\circ}$ and $\gamma_{2}=+20^{\circ}$ following the procedure described in the methods section. Figure 7-3(b) shows example 3D tractography when the EDL muscle was placed at $\alpha=0^{\circ},+20^{\circ}$, and $-30^{\circ}$. Different from the $2 \mathrm{D}$ projected angle shown in Fig. 7-2(b), the 
tractography in Fig. 7-3(b) represented the true 3D fiber orientation in space. These images constructed at different positions $(\alpha)$ were also registered to each other using the same procedure applied in registering the dual-angle images.

Figures 7-3(c) to 7-3(e) show the tissue surface imaged at $\alpha=0^{\circ},+20^{\circ}$, and $-30^{\circ}$ respectively. All these surface contours were from the first position of the dual-angle imaging $\left(\gamma_{1}=-20^{\circ}\right)$. These surface contour patterns showed a significant difference because the EDL muscle was imaged from different angles. However, after rotating the 3D volume back around the axis $\mathbf{V}$ (Fig. 7-3(a)), the resulting surface contours in Figs. 7-3(d) \& 7-3(e) appeared quite similar to the sample surface imaged at $\alpha=0^{\circ}$ (Fig. 7-3(c)). In all cases, 92\% of the surface area had $<5$-pixel difference after rotation, which confirmed the goodness of the image registration.

For the purpose of validation, the directly measured 3D fiber angle at a sample position $\alpha$ was compared with the "expected" angles calculated using 3D fiber angle measured at $\alpha=0^{\circ}$. The "expected" 3D fiber direction $\mathbf{F}^{\mathrm{e}}(\alpha)=\left[f_{\mathrm{x}}(\alpha), f_{\mathrm{y}}(\alpha), f_{\mathrm{z}}(\alpha)\right]^{\mathrm{T}}$ at sample position $\alpha$ can be calculated from the 3D fiber direction $\mathbf{F}(0)$ measured at sample position $\alpha=0$ :

$$
\mathbf{F}^{\mathrm{e}}(\alpha)=\mathbf{R}_{\mathrm{v}}(\alpha) \mathbf{F}(0)=\left[\mathbf{R}_{\mathrm{x}}(-\gamma) \mathbf{R}_{\mathrm{y}}(\alpha) \mathbf{R}_{\mathrm{x}}(\gamma)\right] \mathbf{F}(0)
$$

where $\mathbf{R}_{\mathrm{x}}$ and $\mathbf{R}_{\mathrm{y}}$ are standard rotational matrices around the $\mathrm{x}$ - and $\mathrm{y}$-axis, respectively. To facilitate the comparison among 3D angles, we compared the 2D projected angles of the 3D direction in three orthogonal projection planes calculated in Eq. (7). 
In practice, the 3D angle measured at $\alpha=0^{\circ}$ had experimental errors, which may lead to inaccurate angles calculated at other sample positions. Therefore a "genetic algorithm" based optimization procedure was used to find the best $3 \mathrm{D}$ fiber direction at $\alpha=0^{\circ}$. The original fiber orientation obtained in dual-angle OPT was used as the initial value. True fiber orientation was obtained in the optimization to minimize an error function defined as:

$$
\mathrm{ERR}=\sum_{p} \sum_{i=-30}^{+20}\left|\theta_{p}^{e}\left(\alpha_{0}, \alpha_{i}\right)-\theta_{p}^{m}\left(\alpha_{i}\right)\right|^{2} \quad p \in\{A B, A C, B C\}
$$

where $\theta_{p}^{e}$ stands for the expected fiber angle in the projection plane $p$ calculated by Eq. (9) and $\theta_{p}^{m}$ denotes the measured projection angle from dual-angel OPT at the same projection plane. The optimized 3D fiber angle at sample position of $\alpha=0^{\circ}$ was then used to calculate the expected projection angels at other sample positions.
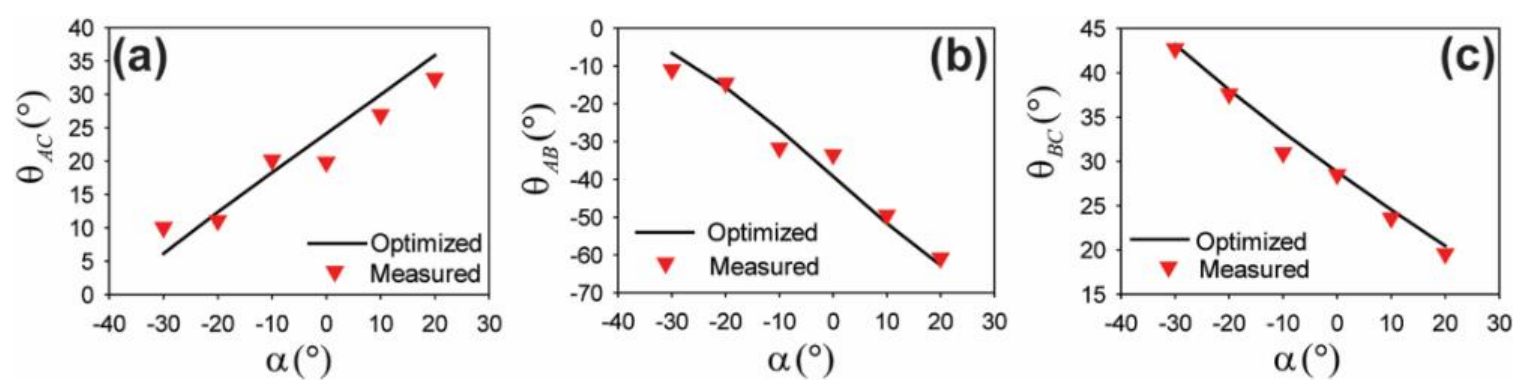

Figure 7-4. Example optimization results. The symbols represented the measured projection angles for a region-of-interest (ROI) in the $\mathrm{AC}(\mathrm{a}), \mathrm{AB}(\mathrm{b})$, and $\mathrm{BC}(\mathrm{c})$ planes at different sample positions. The solid line indicated the optimization results (best-fitted) based on Eq. (10).

Figures 7-4(a-c) show an example of optimization of the fiber orientation measured 
in a $48 \times 48 \times 48 \mu \mathrm{m}^{3}$ region-of-interest (ROI). For this ROI, the polar and azimuthal angles measured from dual-angle OPT at $\alpha=0^{\circ}$ were $73.2^{\circ}$ and $28.7^{\circ}$, respectively; whereas the corresponding optimized angles were $70.8^{\circ}$ and $28.8^{\circ}$. Since the difference in the azimuthal angle was negligible, the overall error (Eq. (10)) was slightly greater in the AC-plane $\left(0.73^{\circ} \pm 2.13^{\circ}\right)$ and $\mathrm{AB}$-plane $\left(-0.25^{\circ} \pm 2.24^{\circ}\right)$ than the BC-plane $\left(0.22^{\circ} \pm 0.98^{\circ}\right)$. Rotating the EDL muscle from $\alpha=-30^{\circ}$ to $\alpha=20^{\circ}$ changed the fiber orientation from $6^{\circ}$ to $35^{\circ}$ in the $\mathrm{AC}$ plane, $-6^{\circ}$ to $-62^{\circ}$ in the $\mathrm{AB}$ plane, and $43^{\circ}$ to $20^{\circ}$ in the $\mathrm{BC}$-plane.
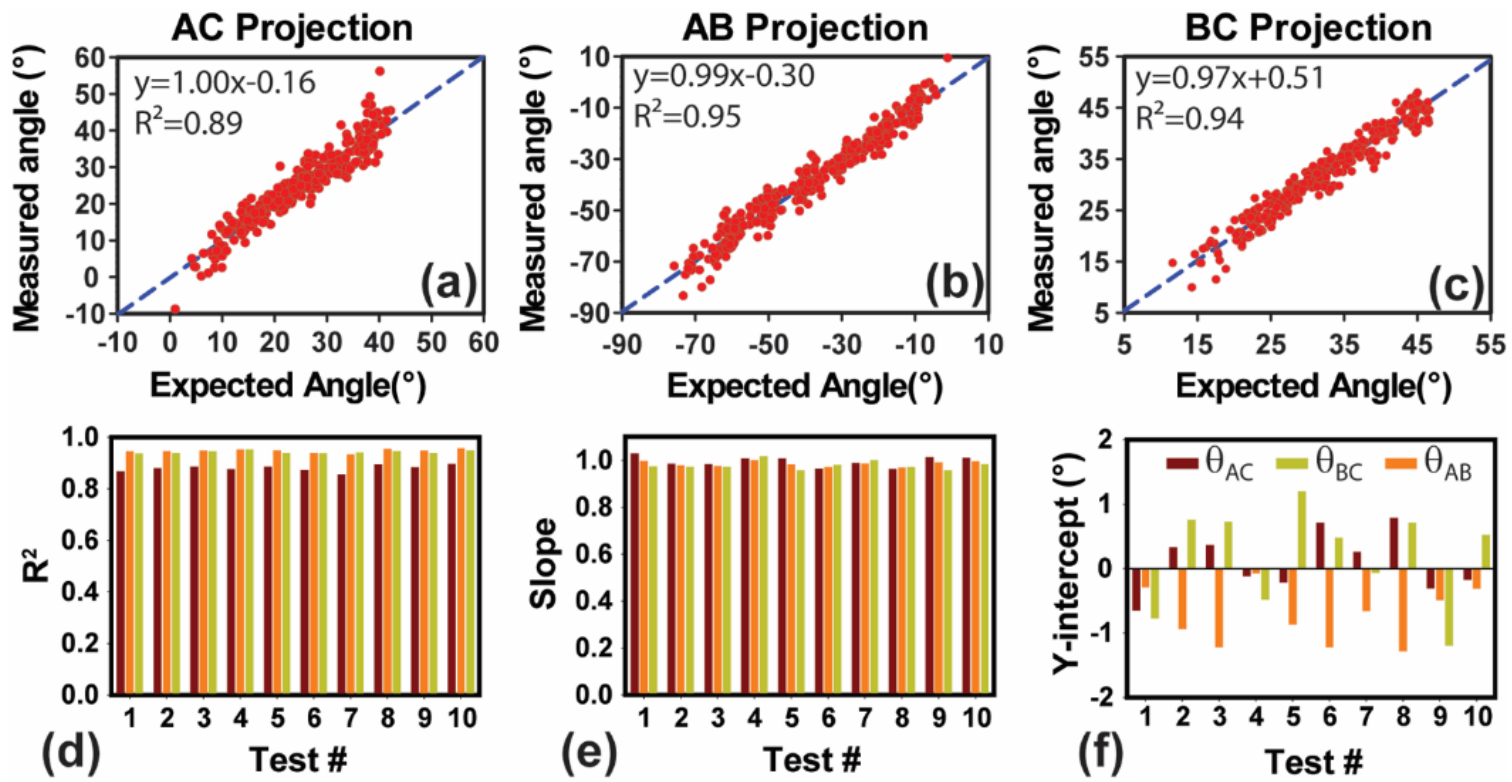

Figure 7-5. The correlation between measured and expected projection angles in fifty randomly selected ROIs $\left(48 \times 48 \times 48 \mu \mathrm{m}^{3}\right)$ from the EDL muscle. The projection angles were calculated in (a) AC-plane, (b) AB-plane, and (c) BC-plane. The regression equation and the coefficient of determination $\left(\mathrm{R}^{2}\right)$ were also shown. The distribution of $(\mathrm{d})$ coefficient of determination $\left(\mathrm{R}^{2}\right)$, (e) slope, and (f) y-intercept of the linear regression analyses between measured and expected fiber angles obtained in ten tests of using 50 random ROIs in the EDL muscle.

Figure 7-5 shows the validation results obtained from 50 randomly selected ROIs 
$\left(\right.$ size $\left.48 \times 48 \times 48 \mu \mathrm{m}^{3}\right)$ in a $0.3 \times 0.3 \times 1.0 \mathrm{~mm}^{3}(\mathrm{~A} \times \mathrm{B} \times \mathrm{C})$ volume of the EDL muscle. Since each ROI was measured at six different sample positions from $\alpha=-30^{\circ}$ to $+20^{\circ}$, there were 300 data points in the correlation analysis between the measured and "expected" projection angles in the AB-, AC- and BC-plane. In all three projection planes, the "expected" angles were highly correlated with the actual measured angles (Fig. 7-5). All three linear regression results showed a close to 1.0 slope and $\leq 0.51^{\circ}$ y-intercept. The coefficients of determination $\left(\mathrm{R}^{2}\right)$ were $0.89,0.95$, and 0.94 in the $\mathrm{AB}-$, $\mathrm{AC}-$, and $\mathrm{BC}$-plane, respectively.

These results suggested that the experimentally measured 3D fiber orientation in dual-angle OPT closely matched with the "expected" results. Among all data points, the mean and standard deviation of the difference between the measured and expected fiber angle were $0.00^{\circ} \pm 3.30^{\circ}, 0.00^{\circ} \pm 4.02^{\circ}$, and $0.14^{\circ} \pm 1.96^{\circ}$ in the $\mathrm{AB}, \mathrm{AC}$, and $\mathrm{BC}$ planes, respectively.

Similar results were obtained when different random sets of ROIs were used in the validation. We repeated ten times the aforementioned procedure of selecting 50 ROIs randomly in the EDL muscle. The mean and standard deviation of the coefficients of determination $\left(\mathrm{R}^{2}\right)$ were $0.88 \pm 0.01,0.95 \pm 0.01$, and $0.94 \pm 0.01$ in the AC-, AB-, and BCplane, respectively (Fig. 7-5(d)). The corresponding slopes of the linear regression were $0.99 \pm 0.02,0.98 \pm 0.01$, and $0.98 \pm 0.02$ (Fig. 7-5(e)); and the y-intercept values were $0.10 \pm 0.46,-0.72 \pm 0.44$, and $0.19 \pm 0.77$ (Fig. 7-5(f)) in the AC-, AB-, and BC-plane respectively. These results indicated a high consistence between the results of dual-angle OPT measurement and the expected values and thus validated dual-angle OPT for 
accurately imaging 3D fiber orientation.

\subsubsection{Tibialis anterior (TA) muscle}

In this section, we applied dual-angle OPT to image the 3D fiber organization in the mouse TA muscle. The muscle was excised from a C57BL/6 mouse and fixed in $4 \%$ paraformaldehyde. The TA sample was imaged at $\gamma_{1}=-25^{\circ}$ and $\gamma_{2}=+25^{\circ}$ in dual-angle OPT.
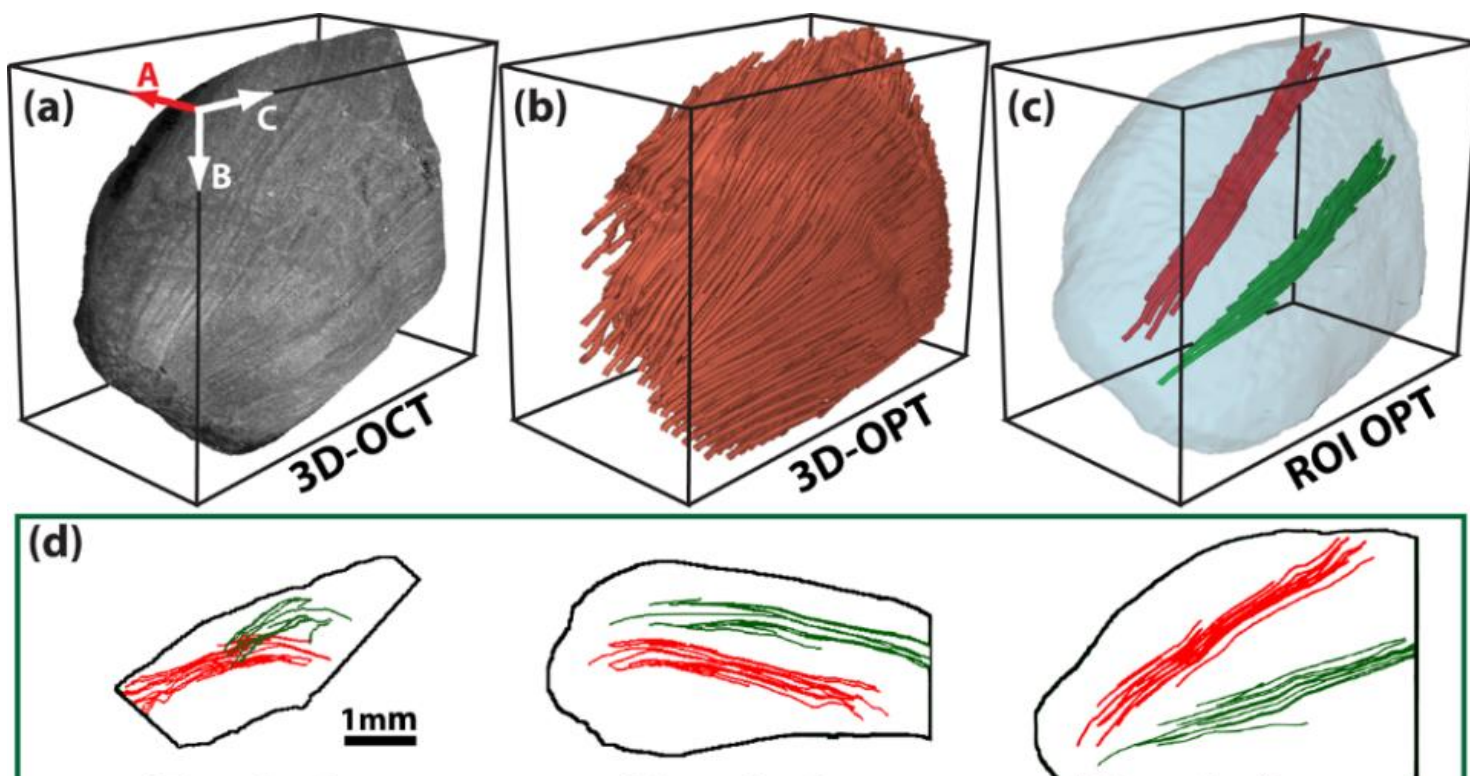

AB projection

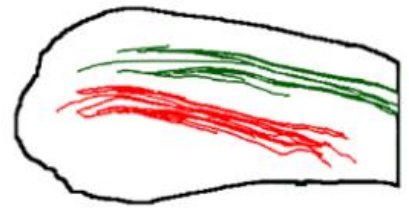

AC projection

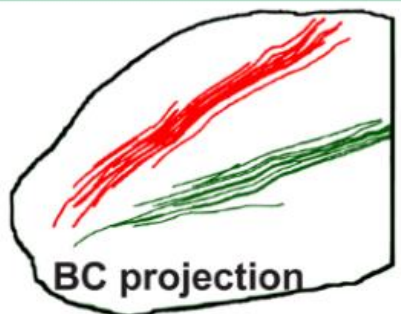

Figure 7-6. Dual-angle OPT of a piece of the mouse tibialis anterior (TA) muscle. (a) The 3D intensity image of the muscle. (b) The 3D tractography obtained in dual-angle OPT. (c) Detailed fiber organization of two separate fiber bundles inside the muscle. (d) The 2D tractography by projecting the $3 \mathrm{D}$ fibers shown in (c) to the $\mathrm{AB}, \mathrm{AC}$, and $\mathrm{BC}$ planes.

Figure 7-6(a) shows the 3D intensity images of the TA muscle imaged at $\gamma_{2}=+25^{\circ}$.

Figure 7-6(b) shows the corresponding 3D tractography constructed in dual-angle OPT.

The 3D tractography indicated that myofibers were mostly aligned along the long axis of 
the TA muscle. Figure 7-6(c) demonstrates the capability of 3D OPT in revealing the detailed fiber architecture by selectively revealing two fiber bundles at two separate locations inside the muscle. As expected, these two fiber bundles were clearly organized along the long axis of the muscle toward the apex. Figure 7-6(d) further demonstrates that the details of the fiber bundles can be studied by projecting the $3 \mathrm{D}$ fiber bundles to $2 \mathrm{D}$ projection planes (the AB-, AC-, and BC-plane).

Because of its large size, the TA muscle myofibers fibers were still discernable in the intensity images. Therefore the intensity images in this case can serve as another validation standard to evaluate the dual-angle OPT. As shown in Fig. 7-6, the 3D fiber orientation obtained in dual-angle OPT can be projected to any $2 \mathrm{D}$ planes in space. For the purpose of this validation, we chose to compare the fiber orientations in the en face (BC) plane. The 3D fiber vectors were projected onto the en face image plane of the sample placed at the second dual-angle position $\left(\gamma_{2}=+25^{\circ}\right)$. The fiber orientations at the same en face plane were also calculated from the corresponding intensity images as described previously (Wang et al. 2014). Briefly, the pixel-wise 2D gradient was calculated using a $3 \times 3$ Sobel window. The distribution of the gradient direction was fitted using the Von Mises distribution within a $200 \times 200 \mu \mathrm{m}^{2}$ evaluation window. The orientation corresponding to the peak of the fitter distribution was assigned as the primary fiber orientation at the center of the evaluation window. 

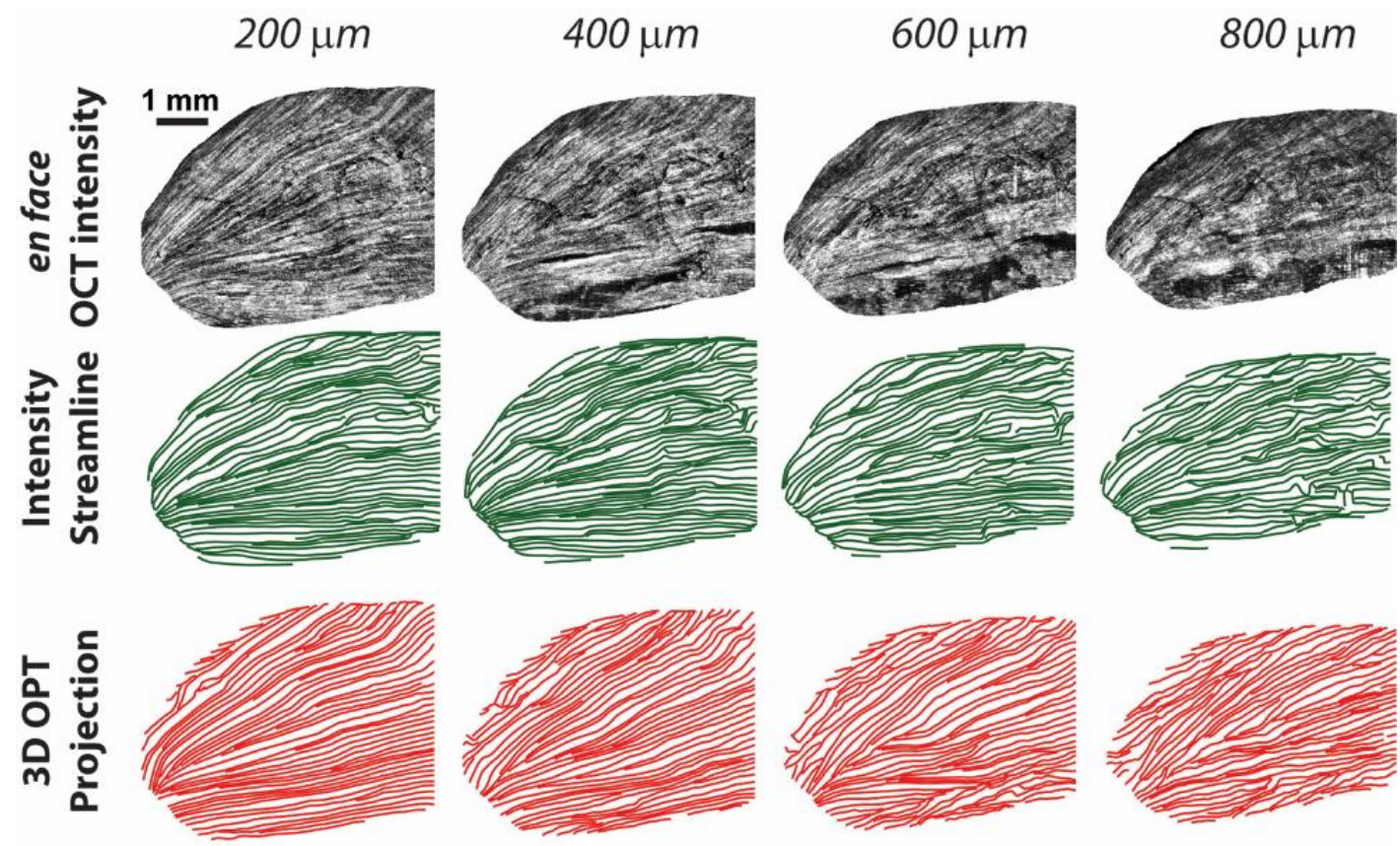

Figure 7-7. A comparison of the fiber organization obtained in dual-angle OPT with that obtained by analyzing OCT intensity profiles in the en face plane at depths from $200 \mu \mathrm{m}$ to $800 \mu \mathrm{m}$. The first row shows the en face intensity obtained in the muscle sample place at $\gamma_{2}=+25^{\circ}$ in dual-angle OPT. The second row shows the tractography calculated from the intensity images. The third row shows the $2 \mathrm{D}$ tractography calculated by projecting the $3 \mathrm{D}$ fiber orientation obtained in dual-angle OPT to the corresponding en face plane (Eq. (7)).

Figure 7-7 shows the comparison of the projection of 3D OPT results with the intensity tractography in the en face plane of the sample imaged at $\gamma_{2}$ at different depths up to $800 \mu \mathrm{m}$. Overall, the dual-angle OPT projection was consistent with intensity based tractography. Myofibers converged along the long muscle axis toward the apex at all depths from the intensity images, which was also evident in the dual-angle OPT results. However, there were also some major discrepancies between these two measurements. The intensitybased tractography appeared to be much noisier, especially at the upper-right part, than the OPT results. The edge-detection based intensity tractography is sensitive to any intensity 
variations. Such variations may not be related to muscle fibers, but can induce artificial fiber orientation changes in the tractography. At depths greater than $\sim 600 \mu \mathrm{m}$, the striated fiber patterns can no longer be clearly resolved in the intensity image. Therefore, the intensity tractography showed larger variations; whereas dual-angle OPT still showed relatively a smooth trend of the fibers toward the apex. When overlaying the OPT results on top of the intensity images, we verified that dual-angle OPT provided a better match with the fiber trajectories than the intensity based method.

\subsubsection{Articular cartilage}

In this section, dual-angle OPT was applied to image the collagen fiber organization in the bovine articular cartilage. The cartilage sample was excised from the middle phalanx and fixed in $4 \%$ paraformaldehyde. This sample was imaged from the cartilage surface (referred to as the "top-scan") using the dual-angle procedures at $\gamma_{1}=-25^{\circ}$ first and then at $\gamma_{2}=+25^{\circ}$.
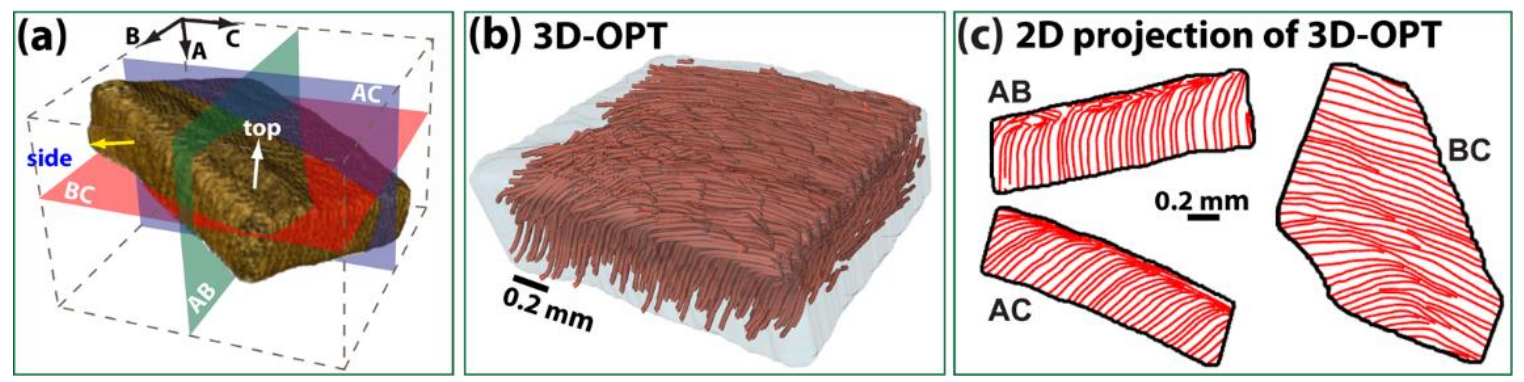

Figure 7-8. 3D visualization of a piece of the bovine cartilage in (a) intensity, (b) 3D fiber tractography from dual-angle measurements, and (c) the 2D tractography by projecting the $3 \mathrm{D}$ fibers shown in (b) to the AB-, AC-, and BC-plane as labeled in (a).

No fibers can be observed from the 3D intensity image of the cartilage (Fig. 7-8(a)). 
However, the constructed 3D fiber tractography from dual-angle OPT (Fig. 7-8(b)) clearly revealed the unique arcade-like fiber organization in the cartilage. In the "superficial" zone, fibers were oriented relatively parallel to the sample surface. Then the fibers arched in the "transitional" zone to be eventually perpendicular toward the boundary between noncalcified and calcified cartilage in the deeper "radial" zones. Moreover, the 3D tractography correctly revealed the "brushing" direction, i.e. all fibers were bended toward the same direction (Lu et al. 2014).

Similar to the example of the TA muscle (Fig. 7-7), the 3D OPT data set can be explored programmingly to provide a detailed view of the fiber architecture. Due to the unique "arcade" 3D fiber structure in the cartilage, the fiber orientation would appear differently when viewed from different angles or in cutting planes made at different orientations. Figure 7-8(c) demonstrated such an effect by showing the fiber organization when projecting the $3 \mathrm{D}$ orientation to three orthogonal 2D cutting planes (Eq. 7). This result underscored the importance of considering the cutting directions when evaluating the histology and scanning electron microscopy (SEM) images of cartilage samples (Clark 1990).

To verify the obtained 3D fiber architecture, one side of the cartilage (indicated in Fig. 7-8(a)) was directly imaged using OPT. The intensity image (Fig. 7-9(a)) showed the boundary between the non-calcified and calcified cartilage, but no fiber structure can be observed. The resulted 2D tractography (Fig. 7-9(a)) clearly uncovered the arcade-like fiber structure that was conventionally used to identify three different zones in the cartilage 
(Clark 1990; Xia et al. 2001; Lee \& Xia 2013). The fiber appeared to be randomized in the calcified region. As a comparison, the 3D fiber orientation data measured at the same location using dual-angle OPT were projected on the same side-scan plane. Although this plane was parallel to the incident light direction or perpendicular to the OPT evaluation plane (Fig. 7-1), the projected 2D tractography (Fig. 7-9(b)) appeared qualitatively in good agreement with the directly measured "side-scan" result (Fig. 7-9(a)).
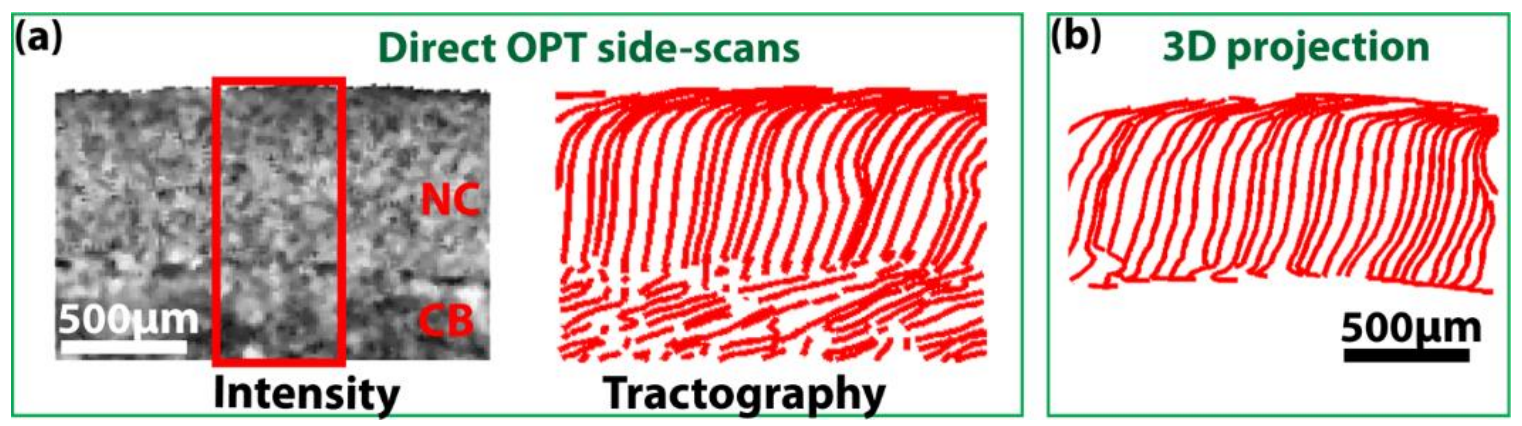

Figure 7-9. (a) The en face intensity and tractography images obtained from direct OPT imaging of the side of the cartilage sample (the "side-scan" as labeled in Fig. 7-8). (b) The tractography by projecting the 3D fiber orientation obtained in dual-angle OPT to the same tissue side as imaged in (a). NC: non-calcified cartilage; $\mathrm{CB}$ : calcified cartilage/bone.

We further quantitatively compared the "side-scan" results with the 3D projection. Figure 7-10(a) shows the depth-dependent fiber orientation obtained by averaging all linescans within a ROI (marked with a red box in Fig. 7-9(a)). The "transitional" segment where the fiber orientation changed from parallel to perpendicular can be well fitted (adjusted $\mathrm{R}^{2}=0.999$ ) using a hyperbolic tangent function as reported in a previous study using polarization light microscopy (PLM) (Xia et al. 2001). The fitted equation, y = 42.06 $\times \tanh [(\mathrm{x}-121.8) / 54.47]+46.13$, was plotted as a blue line in Fig. 7-10(a). The first 
derivative of the fitting equation was shown as the dark-green curve. The boundaries of the transitional zone were determined as the boundary positions of the full-width-halfmaximum (FWHM) of the first derivative curve (Lee \& Xia 2013). This procedure resulted in $\sim 74 \mu \mathrm{m}$ as the boundary between the superficial and transitional zone, and $\sim 170 \mu \mathrm{m}$ as the boundary between the transitional and the radial zone.
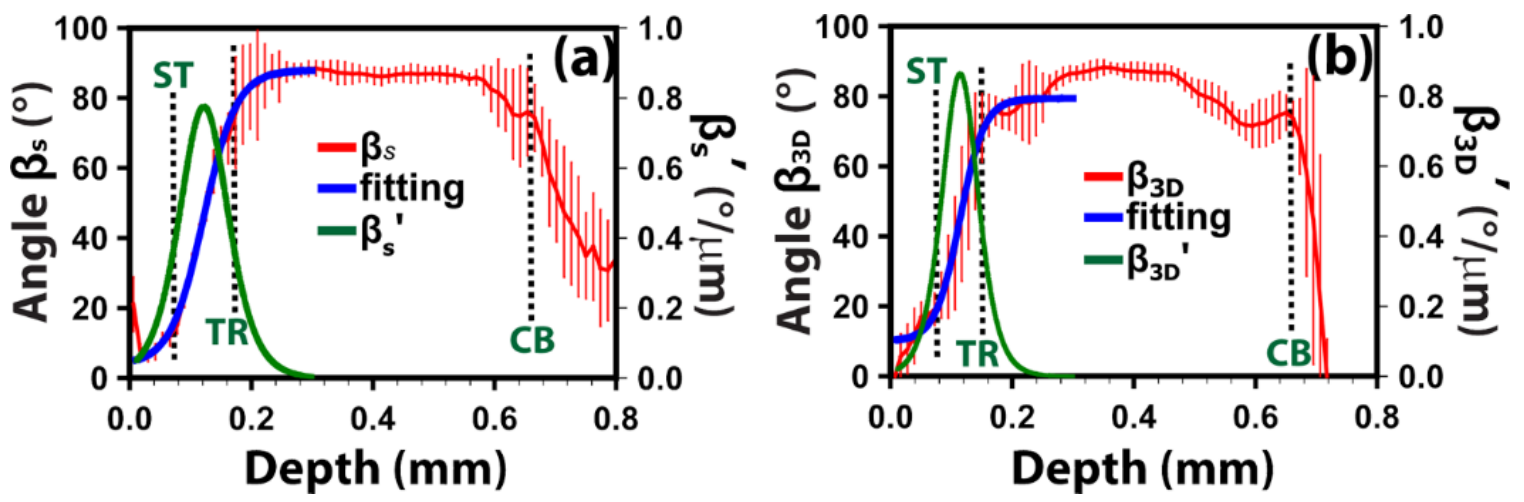

Figure 7-10. (a) The fiber orientation over the depth obtained from the side-scan image by averaging all B-scan inside a $0.5 \mathrm{~mm}$ wide ROI (marked in red box in Fig. 7-9(a)).(b) The orientation change over depth obtained from the projected 2D fiber orientation (Fig. 79(b)). The blue lines in (a) and (b) are fitting results using a hyperbolic tangent function. The green curves are the first order derivative of the curve fitting. The labels "ST", "TR", and "CB" indicate the boundaries between the superficial and transitional zone, the transitional and radial zone, and cartilage and bone, respectively. Error bars indicate standard deviation.

The above procedure was repeated using the projected angle $\theta_{\mathrm{p}}$ calculated from the 3D fiber orientation using dual-angle OPT (Fig. 7-9(b)). We used $n=1.5$ as the optical refractive index of the cartilage as reported in a previous study (Herrmann et al. 1999). The orientation profile can also be well fitted (adjusted $\mathrm{R}^{2}=0.989$ ) using a hyperbolic tangent function: $y=34.69 \times \tanh [(x-113.7) / 40.14]+44.7$. Using the same criteria described in the previous paragraph, the boundary between the superficial zone and the transitional zone 
was located at $\sim 79 \mu \mathrm{m}$ which was consistent with that determined from direct side-scan. The boundary between the transitional zone and the radial zone was at $\sim 150 \mu \mathrm{m}$ which was slightly smaller than that determined from the side-scan. It was noticed that the fiber orientation had larger fluctuation at the junction between the transitional zone and the radial zone, which may have caused larger measurement errors in both cases. Similar observation has been reported previously using PLM (Xia et al. 2001), which was attributed to fiber variation in the transitional zone.

The directly measured side-scan results (Fig. 7-10(a)) indicated that the orientation started to decrease significantly at $\sim 660 \mu \mathrm{m}$. Similarly, the projected angle from 3D

measurements also showed a significant decrease in fiber orientation at $\sim 656 \mu \mathrm{m}$. This location was likely the boundary between the non-calcified cartilage and calcified cartilage or bone. The fiber orientation also had large variations within this region, which may explain the difference between the two measurements within this region. At locations inside the bone region, the side-scan results showed better imaging depth than the 3D projection results.

\subsection{Discussions}

Imaging 3D fiber organization is important to understand mechanical properties and normal physiological functions in fibrous tissues. In this study, 3D fiber orientation was successfully imaged by using a dual-angle OPT procedure. The proposed method utilizes tissue optical polarization properties to calculate actual 3D fiber orientations. The accuracy 
and performance of dual-angle OPT were investigated by testing three different biological tissues. These studies indicated that dual-angle OPT can reliably acquire the full 3D images of microscopic fiber organization in complicated biological tissues.

The EDL muscle was used in the validation test due to its uniform and wellorganized fiber structure. The experiment was carefully designed to ensure the rotation axis and the amount of rotation can induce sufficient changes in fiber orientation. The quantitative comparison between the expected and the experimental values showed consistent accuracy of dual-angle OPT. The two images acquired in the dual-angle test were accurately registered at each sample position. In addition, the sample images acquired at multiple different rotational positions were also registered to the reference location at $\alpha=0^{\circ}$. However, it is challenging to achieve a perfect registration in all 12 images used in the validation. We noticed that a couple of distant data points had relatively large errors in the regression results (Fig. 7-5). A close examination revealed that these points were part of the dual-angle imaging at the two largest rotational positions at $\alpha=+20^{\circ}$ and $\alpha=-30^{\circ}$. This error was likely caused by the large surface inclination at these sample positions. A larger light incident angle induced bigger beam deviation, which was more sensitive to slight errors in image registration.

Although the measured and expected fiber orientations were highly correlated in all three orthogonal projection planes, the correlation in the $\mathrm{AC}$ projection plane was marginally poorer than in the other two planes (Fig. 7-5). We believe this can be attributed to two factors after analyzing the optimization results obtained in all ROIs, First, we found 
that the measured polar angle of the $3 \mathrm{D}$ fibers had a very small $\left(3.15^{\circ} \pm 1.75^{\circ}\right)$ but consistent difference from the optimized results; whereas the corresponding azimuthal angle had better agreement with the optimized values $\left(1.60^{\circ} \pm 1.57^{\circ}\right)$. As shown in Eq. 7 , a larger variation in the polar angle $\theta_{f}$ affected the z-axis related projection planes (AB- and ACplane) more than the BC-plane. Such a slightly bigger variation in the polar angle was likely due to an imperfect alignment of the rotational axis. Second, it is known that a similar amount of error in the regression model may lead to a smaller $\mathrm{R}^{2}$ if the measurements had a smaller range. Fiber orientations changed much greater in the $\mathrm{AB}$-plane $\left(\sim 80^{\circ}\right)$ than in the projection planes associated with the $\mathrm{x}$-axis $\left(\sim 40^{\circ}\right.$ changes in the $\mathrm{AC}$ - and $\mathrm{BC}$-plane). Therefore the measurements in the AC-plane were affected by both factors as discussed above, which resulted in a slightly worse correlation.

The TA muscle was used as another validation because the muscle fibers were discernable in the intensity images as well. This is because the muscle fibers were bigger in the TA muscle than in the EDL muscle as revealed in a histology study. In general, the fiber orientation obtained in dual-angle OPT was consistent with that obtained using intensity based image processing. Such a direct comparison also revealed some important advantages in the OPT method. In order to resolve fibers using intensity based contrast, the image resolution should be sufficiently high to detect intensity changes around the fibers or fiber bundles. Then image processing methods were commonly applied within an evaluation window to determine the primary orientation inside the window. The size of the evaluation window sometimes reached a few hundred-micrometers (Goergen et al. 2012). 
Therefore, the actually spatial resolution was compromised in computing the fiber orientation. In addition, the intensity based image processing may be affected by any intensity changes that are not related to fibers. For example, OCT intensity images may contain the "banding" artifacts in a birefringent tissue caused by the residual polarization effects in the imaging system (Yang et al. 2014). Such non-fiber related structural changes may cause incorrect fiber calculations. This issue became more severe at large depths when intensity contrast deteriorated as shown in Fig. 7-7.

Intensity-based tractography was commonly applied to the en face images (Fleming et al. 2008; Goergen et al. 2012), and thus it can obtain the projected fiber orientation in the en face plane. A computational approach was previously explored to calculate the 3D fiber orientation from the measured 2D orientation in the en face plane (Gan \& Fleming 2013). It relied on the assumption that the fibers were parallel to the sample surface, which is not generally applicable for most fibrous tissues including heart, cartilage, and neural tissues. A structure tensor based algorithm was recently reported to measure 3D fiber orientation by analyzing the 3D intensity variation (Wang et al. 2015a). However, this approach has the same drawback as in any intensity based methods as described above.

As a comparison, OPT can maintain the native spatial resolution of the imaging system. It calculates fiber orientation using the local optic axis on a single-pixel basis. The optic axis calculation in OPT is not affected by structural variations in the intensity image. OPT does not require a spatial resolution that is high enough to resolve individual fibers or fiber bundles. In fact, the intensity images failed to reveal any fibers in both the EDL 
muscle and bovine cartilage. However, OPT still successfully imaged the local fiber orientation in these samples, which represented the averaged fiber orientation at each image pixel. The dual-angle OPT procedure described in this work relied upon basic geometrical transformations and did not need any presumptuous information regarding the fiber structure. The results shown in Fig. 7-7 supported that OPT worked better than intensitybased method especially at larger imaging depths.

The articular cartilage results best demonstrated the advantage of the proposed dual-angle OPT. Articular cartilage is structurally reinforced by a network of collagen fibers which are organized in a special "arcade" architecture (Clark 1990; Lu et al. 2014). A single-scan OPT can still map the fiber orientation in the cartilage similar to imaging applications in other tissue samples (Wang \& Yao 2013; Azinfar et al. 2015; Wang et al. 2015b). However, single-scan OPT measures the projected fiber angle in the 2D evaluation plane as illustrated in Fig. 7-1. These angles measured in single-scan OPT may correctly represent the fiber orientation in the superficial cartilage where collagen fibers are tangential to the sample surface. The fibers are almost perpendicular to the surface or aligned with the incident light in the deep radial zone of the cartilage. Therefore the measured 2D projection angles in the radial zone do not represent the complete picture of the true $3 \mathrm{D}$ fiber orientation there.

Dual-angle OPT clearly revealed the well-known arcade-like fiber organization in the cartilage. The obtained 3D fiber orientation can be projected to the $2 \mathrm{D}$ side plane of the cartilage and compared with the directly measured fiber orientation. Despite the challenge 
of achieving a perfect image registration, the projected fiber orientation is consistent with the directly measured fiber orientation. The unique fiber organization in the cartilage plays a critical role in maintaining its proper mechanical properties. Changes in collagen fiber structure including the orientation are signs of early cartilage degeneration (Shim et al. 2016). Therefore imaging fiber organization in cartilage may be useful for detecting early cartilage diseases. Polarization light microscopy (PLM) (Xia et al. 2001; Lee \& Xia 2013) is currently the standard way for imaging fiber orientation in cartilage. Unfortunately, PLM can only image thin cartilage slices due to its limited imaging depth. Being able to map the true 3D fiber structure in cartilage, dual-angle OPT provides a better nondestructive alternative to PLM and may find many valuable applications in cartilage studies.

The geometric transformations used in dual-angle OPT rely on the local fiber orientation measured in each individual OPT as well as the orientation and location of the corresponding "evaluation plane" (Fig. 7-1). It is important to note that the "cumulative" optic axis measured in conventional PSOCT studies does not represent the correct fiber orientation in tissue (Fan \& Yao 2012c) and cannot be used for constructing the 3D orientation. The surface refraction changes the orientation and location of the evaluation plane (Fig. 7-1). Although its effect may not be significant at small incident angles or in relatively homogeneous samples, correcting its effect can ensure a proper image registration for dual-angle calculation in general situation. The impact of refraction correction was observed in the validation results using EDL muscle due to the large fiber orientation changes induced by sample rotation. Following the exact same procedures, but 
using a refractive index of 1.0, we found that the correlation results shown in Fig. 7-5 were negatively affected especially in the $\mathrm{BC}$ projection where the correlation $\mathrm{R}^{2}$ decreased to 0.79 , the fitting slope decreased to 0.74 , and the intercept increased to $8.99^{\circ}$.

The surface refraction may also introduce a polarization change because of the different transmission in the s- and p-polarized components. As an example, such a transmission difference is $\sim 3.3 \%$ at a $30^{\circ}$ incident angle and 1.5 sample refractive index based on Fresnel's equation. Because this small difference introduced a $<1^{\circ}$ change in polarization orientation, it was not considered in the current study. The current refraction correction method may require a relatively smooth sample surface. For tissues with rough surface, refractive index matching solution might be used to circumvent the need of refraction correction.

It may be beneficial to use a small incident angle as a large incident angle may also affect the effective imaging depth. For example, a 1-mm imaging depth may be reduced to $866 \mu \mathrm{m}$ with a $30^{\circ}$ refractive angle due to oblique optical path. Dual-angle OPT only calculates the fiber orientation in the common image volumes acquired at two different sample positions. Therefore its effective image depth is limited by the bigger refractive angle between the two volumes. In this study, the imaging depth was greater than $800 \mu \mathrm{m}$ in the TA muscle and $\sim 700 \mu \mathrm{m}$ in the cartilage sample when $\pm 25^{\circ}$ incident angles were used in the dual-angle measurements. Limiting the incident angle can also reduce the aforementioned refraction induced polarization changes. Nevertheless, using a light source at a longer wavelength (e.g. $1.3 \mu \mathrm{m})$ may achieve greater imaging depths in each single- 
scan OPT and thus can extend the overall effective imaging depth in dual-angle OPT.

Finally, the need of two images from the same sample acquired at different angles might be challenging for in vivo applications because sample movement may reduce the size of the common volume used in dual-angle reconstruction. Therefore, innovative system designs that can achieve simultaneous dual-angle scanning might be desirable for in vivo imaging.

\subsection{Conclusion}

We proposed a method to nondestructively image 3D fiber orientation in a tissue. This method utilized the recently developed OPT method at two different imaging angles in relation to the sample. Each OPT measured the projected $2 \mathrm{D}$ orientation within a different evaluation plane. The 3D fiber orientation was then constructed using geometrical transformation based on the two measurements. We described an example implementation of this method and conducted several tests to verify the obtained $3 \mathrm{D}$ angles in several different biological tissues. In particular, dual-angle OPT successfully imaged the unique depth-varying fiber orientation in cartilage. As an enabling tool, dual-angle OPT provides a promising platform for studying the important 3D fiber architecture in many fibrous tissues. 


\section{Chapter 8. Summary and future direction}

In Summary, this dissertation research had three major contributions to the development of the OPT technology. First, a comprehensive histology validation study was conducted to verify the accuracy of OPT imaging results. Second, the capability of OPT was demonstrated by imaging heart and skeletal muscle samples of the $m d x$ mice. Third, a dualangle OPT method was developed to image the absolute 3D fiber orientation in tissue.

The comprehensive validation study presented in Chapter 2 has established the accuracy of using OPT for imaging depth-resolved fiber orientation in tissue. Chapter 3 then illustrated OPT's capability to visualize the 3D structure in mouse heart. We successfully demonstrated that OPT can reveal the global fiber structural changes (Chapter 4) and identify the micro-level fiber disorganization in the $m d x$ hearts (Chapter 5). In addition, OPT can visualize and quantify muscle damages in the skeletal muscle of the mdx mice (Chapter 6). In comparison with conventional histological analysis, OPT provides a fast and non-destructive way to visualize the microscopic fiber disorganization in a 3D tissue volume. Therefore it is potentially helpful in detection and studying the disease related tissue damage at early stages when the fiber structural changes are focal and small. Chapter 7 demonstrated that dual-angle OPT can successfully image the absolute tissue 3D fiber orientation. This is valuable for studying fibrous tissue with complicated 3D architecture such as the neural fibers in brain and collagen structure in articular cartilage. 
Overall, this dissertation research clearly demonstrated the value of OPT as a practical imaging tool to accurately map the fiber organization. As an enabling tool, OPT can be applied to image a variety of fibrous tissues beyond hearts and skeletal muscles that have been used in this research. For example, imaging neural fibers may be an important application to study the interconnected neural network in the brain. Further development of the OPT technology to enable in vivo and endoscopic applications will significantly enhance this technology's potential for a greater range of research and clinical applications. 


\section{References}

Agger P., Lakshminrusimha S., Laustsen C., Gugino S., Frandsen J.R., Smerup M., Anderson R.H., Hjortdal V. \& Steinhorn R.H. (2016) The myocardial architecture changes in persistent pulmonary hypertension of the newborn in an ovine animal model. Pediatr Res 79, 565-74.

Alan E. H. Emery F.M., and Rosaline C. M. Quinlivan (2003) Duchenne Muscular Dystrophy. Oxford University Press.

Ambrosi C.M., Fedorov V.V., Schuessler R.B., Rollins A.M. \& Efimov I.R. (2012) Quantification of fiber orientation in the canine atrial pacemaker complex using optical coherence tomography. Journal of Biomedical Optics 17, 071309.

Azinfar L., Ravanfar M., Wang Y., Zhang K., Duan D. \& Yao G. (2015) High resolution imaging of the fibrous microstructure in bovine common carotid artery using optical polarization tractography. Journal of Biophotonics, n/a-n/a.

Barbin I.C.C., Pereira J.A., Bersan Rovere M., de Oliveira Moreira D., Marques M.J. \& Santo Neto H. (2016) Diaphragm degeneration and cardiac structure in mdx mouse: Potential clinical implications for Duchenne muscular dystrophy. Journal of Anatomy 228, 784-91.

Basser P.J., Mattiello J. \& LeBihan D. (1994) MR diffusion tensor spectroscopy and imaging. Biophysical journal 66, 259-67.

Benoist D., Stones R., Drinkhill M.J., Benson A.P., Yang Z., Cassan C., Gilbert S.H., Saint D.A., Cazorla O., Steele D.S., Bernus O. \& White E. (2012) Cardiac arrhythmia mechanisms in rats with heart failure induced by pulmonary hypertension. American Journal of Physiology - Heart and Circulatory Physiology 302, H2381-H95.

Benson A.P., Gilbert S.H., Li P., Newton S.M. \& Holden A.V. (2008) Reconstruction and quantification of diffusion tensor imaging-derived cardiac fibre and sheet structure in ventricular regions used in studies of excitation propagation. Mathematical Modelling of Natural Phenomena 3, 101-30. 
Bostick B., Yue Y., Lai Y., Long C., Li D. \& Duan D. (2008a) Adeno-Associated Virus Serotype-9 Microdystrophin Gene Therapy Ameliorates Electrocardiographic Abnormalities in mdx Mice. Human Gene Therapy 19, 851-6.

Bostick B., Yue Y., Long C. \& Duan D. (2008b) Prevention of dystrophin-deficient cardiomyopathy in twenty-one-month-old carrier mice by mosaic dystrophin expression or complementary dystrophin/utrophin expression. Circulation research 102, 121-30.

Bostick B., Yue Y., Long C., Marschalk N., Fine D.M., Chen J. \& Duan D. (2009) Cardiac Expression of a Mini-dystrophin That Normalizes Skeletal Muscle Force Only Partially Restores Heart Function in Aged Mdx Mice. Molecular Therapy: the Journal of the American Society of Gene Therapy 17, 253-61.

Breckenridge R. (2010) Heart failure and mouse models. Disease Models and Mechanisms 3, 138-43.

Buckberg G., Hoffman J.I.E., Mahajan A., Saleh S. \& Coghlan C. (2008) Cardiac Mechanics Revisited. The Relationship of Cardiac Architecture to Ventricular Function 118, 2571-87.

Cheng Y.J., Lang D., Caruthers S.D., Efimov I.R., Chen J. \& Wickline S.A. (2012) Focal but reversible diastolic sheet dysfunction reflects regional calcium mishandling in dystrophic mdx mouse hearts. American Journal of Physiology - Heart and Circulatory Physiology 303.

Ciszek B., Skubiszewska D. \& Ratajska A. (2007) The anatomy of the cardiac veins in mice. Journal of Anatomy 211, 53-63.

Clark J.M. (1990) The organisation of collagen fibrils in the superficial zones of articular cartilage. Journal of Anatomy 171, 117-30.

Clayton R.H., Abdalhamid S., Bloor R., Kyprianou G., Kotagiri K., Lee J., Mane A. \& White R. (2010) Transmural changes in fibre helix angle in normal and failing canine ventricles. pp. 915-8.

Cohn J.N., Ferrari R. \& Sharpe N. (2000) Cardiac remodeling-concepts and clinical implications: A consensus paper from an International Forum on Cardiac Remodeling. Journal of the American College of Cardiology 35, 569-82. 
Cui W., Jang A., Zhang P., Thompson B., Townsend D., Metzger J.M. \& Zhang J. (2015) Early detection of myocardial bioenergetic deficits: A 9.4 tesla complete non invasive 31P MR spectroscopy study in mice with muscular dystrophy. PLoS ONE 10.

De Bruin P.F., Ueki J., Bush A., Khan Y., Watson A. \& Pride N.B. (1997) Diaphragm thickness and inspiratory strength in patients with Duchenne muscular dystrophy. Thorax 52, 472-5.

Doevendans P.A., J. Daemen M., de Muinck E.D. \& Smits J.F. (1998) Cardiovascular phenotyping in mice. Cardiovascular Research 39, 34-49.

Duan D. (2006) Challenges and opportunities in dystrophin-deficient cardiomyopathy gene therapy. Human Molecular Genetics 15, R253-R61.

Dunn J.F. \& Zaim-Wadghiri Y. (1999) Quantitative magnetic resonance imaging of the mdx mouse model of duchenne muscular dystrophy. Muscle and Nerve 22, 136771.

Emery A.E.H. \& Muntoni F. (2003) Duchenne Muscular Dystrophy. Oxford University Press.

Facon D., Ozanne A., Fillard P., Lepeintre J.F., Tournoux-Facon C. \& Ducreux D. (2005) MR diffusion tensor imaging and fiber tracking in spinal cord compression. American Journal of Neuroradiology 26, 1587-94.

Fan C. \& Yao G. (2010a) Correcting optical-axis calculation in polarization-sensitive optical coherence tomography. IEEE Transactions on Biomedical Engineering 57, 2556-9.

Fan C. \& Yao G. (2010b) Single camera spectral domain polarization-sensitive optical coherence tomography using offset B-scan modulation. Optics Express 18, 72817.

Fan C. \& Yao G. (2012a) Full-range spectral domain Jones matrix optical coherence tomography using a single spectral camera. Optics Express 20, 22360-71.

Fan C. \& Yao G. (2012b) Mapping local optic axis in birefringent samples using polarization-sensitive optical coherence tomography. Journal of Biomedical Optics 17. 
Fan C. \& Yao G. (2012c) Mapping local retardance in birefringent samples using polarization sensitive optical coherence tomography. Optics Letters 37, 1415-7.

Fan C. \& Yao G. (2013) Imaging myocardial fiber orientation using polarization sensitive optical coherence tomography. Biomedical Optics Express 4, 460-5.

Fayssoil A., Renault G., Guerchet N., Marchiol-Fournigault C., Fougerousse F. \& Richard I. (2013) Cardiac characterization of mdx mice using high-resolution doppler echocardiography. Journal of Ultrasound in Medicine 32, 757-61.

Fenton F. \& Karma A. (1998) Vortex dynamics in three-dimensional continuous myocardium with fiber rotation: Filament instability and fibrillation. Chaos $\mathbf{8}, 20-$ 47.

Fleming C.P., Ripplinger C.M., Webb B., Efimov I.R. \& Rollins A.M. (2008) Quantification of cardiac fiber orientation using optical coherence tomography. Journal of Biomedical Optics 13.

Froeling M., Oudeman J., Strijkers G.J., Maas M., Drost M.R., Nicolay K. \& Nederveen A.J. (2014) Muscle Changes Detected with Diffusion-Tensor Imaging after LongDistance Running. Radiology 274, 548-62.

Gan Y. \& Fleming C.P. (2013) Extracting three-dimensional orientation and tractography of myofibers using optical coherence tomography. Biomedical Optics Express 4, 2150-65.

Gilbert S.H., Benson A.P., Li P. \& Holden A.V. (2007) Regional localisation of left ventricular sheet structure: integration with current models of cardiac fibre, sheet and band structure. European Journal of Cardio-thoracic Surgery 32, 231-49.

Goergen C.J., Radhakrishnan H., Sakadžix́ S., Mandeville E.T., Lo E.H., Sosnovik D.E. \& Srinivasan V.J. (2012) Optical coherence tractography using intrinsic contrast. Optics Letters 37, 3882-4.

Hakim C.H., Grange R.W. \& Duan D. (2011) The passive mechanical properties of the extensor digitorum longus muscle are compromised in 2-to 20-mo-old mdx mice. Journal of Applied Physiology 110, 1656-63.

Healy L.J., Jiang Y. \& Hsu E.W. (2011) Quantitative comparison of myocardial fiber structure between mice, rabbit, and sheep using diffusion tensor cardiovascular magnetic resonance. Journal of Cardiovascular Magnetic Resonance $\mathbf{1 3 .}$ 
Helm P.A., Younes L., Beg M.F., Ennis D.B., Leclercq C., Faris O.P., McVeigh E., Kass D., Miller M.I. \& Winslow R.L. (2006) Evidence of structural remodeling in the dyssynchronous failing heart. Circulation Research 98, 125-32.

Herrmann J.M., Pitris C., Bouma B.E., Boppart S.A., Jesser C.A., Stamper D.L., Fujimoto J.G. \& Brezinski M.E. (1999) High resolution imaging of normal and osteoarthritic cartilage with optical coherence tomography. Journal of Rheumatology 26, 627-35.

Hewing B. \& Fisher E.A. (2012) Preclinical mouse models and methods for the discovery of the causes and treatments of atherosclerosis. Expert Opinion on Drug Discovery 7, 207-16.

Hitzenberger C.K., Götzinger E., Sticker M., Pircher M. \& Percher A.F. (2001) Measurement and imaging of birefringence and optic axis orientation by phase resolved polarization sensitive optical coherence tomography. Optics Express 9 , 780-90.

Hoffman E.P., Brown Jr R.H. \& Kunkel L.M. (1987) Dystrophin: The protein product of the duchenne muscular dystrophy locus. Cell 51, 919-28.

Hsu E.W., Muzikant A.L., Matulevicius S.A., Penland R.C. \& Henriquez C.S. (1998) Magnetic resonance myocardial fiber-orientation mapping with direct histological correlation. American Journal of Physiology - Heart and Circulatory Physiology 274, H1627-H34.

Huang D., Swanson E.A., Lin C.P., Schuman J.S., Stinson W.G., Chang W., Hee M.R., Flotte T., Gregory K., Puliafito C.A. \& Fujimoto J.G. (1991) Optical coherence tomography. Science 254, 1178-81.

Humbertclaude V., Hamroun D., Bezzou K., Bérard C., Boespflug-Tanguy O., Bommelaer C., Campana-Salort E., Cances C., Chabrol B., Commare M.-C., Cuisset J.-M., de Lattre C., Desnuelle C., Echenne B., Halbert C., Jonquet O., Labarre-Vila A., N'Guyen-Morel M.-A., Pages M., Pepin J.-L., Petitjean T., Pouget J., Ollagnon-Roman E., Richelme C., Rivier F., Sacconi S., Tiffreau V., Vuillerot C., Picot M.-C., Claustres M., Béroud C. \& Tuffery-Giraud S. (2012) Motor and respiratory heterogeneity in Duchenne patients: Implication for clinical trials. European Journal of Paediatric Neurology 16, 149-60.

Jiang Y., Pandya K., Smithies O. \& Hsu E.W. (2004) Three-dimensional diffusion tensor microscopy of fixed mouse hearts. Magnetic Resonance in Medicine 52, 453-60. 
Jiao S., Todorović M., Stoica G. \& Wang L.V. (2005) Fiber-based polarization-sensitive Mueller matrix optical coherence tomography with continuous source polarization modulation. Applied Optics 44, 5463-7.

Karlon W.J., Covell J.W., McCulloch A.D., Hunter J.J. \& Omens J.H. (1998) Automated measurement of myofiber disarray in transgenic mice with ventricular expression of ras. Anatomical Record 252, 612-25.

Kasaragod D.K., Lu Z., Jacobs J. \& Matcher S.J. (2012) Experimental validation of an extended Jones matrix calculus model to study the 3D structural orientation of the collagen fibers in articular cartilage using polarization-sensitive optical coherence tomography. Biomedical Optics Express 3, 378-87.

Kemp N.J., Zaatari H.N., Park J., Rylander Iii H.G. \& Milner T.E. (2005) Depth-resolved optic axis orientation in multiple layered anisotropic tissues measured with enhanced polarization-sensitive optical coherence tomography (EPS-OCT). Optics Express 13, 4507-18.

Kido M., Otani H., Kyoi S., Sumida T., Fujiwara H., Okada T. \& Imamura H. (2004) Ischemic preconditioning-mediated restoration of membrane dystrophin during reperfusion correlates with protection against contraction-induced myocardial injury. American Journal of Physiology - Heart and Circulatory Physiology 287, H81-H90.

Kim J., Kim D.C. \& Back S.H. (2009) Demonstration of high lateral resolution in laser confocal microscopy using annular and radially polarized light. Microscopy Research and Technique 72, 441-6.

Kléber A.G. \& Rudy Y. (2004) Basic Mechanisms of Cardiac Impulse Propagation and Associated Arrhythmias. Physiological Reviews 84, 431-88.

Klyen B.R., Armstrong J.J., Adie S.G., Radley H.G., Grounds M.D. \& Sampson D.D. (2008) Three-dimensional optical coherence tomography of whole-muscle autografts as a precursor to morphological assessment of muscular dystrophy in mice. Journal of Biomedical Optics 13.

Klyen B.R., Scolaro L., Shavlakadze T., Grounds M.D. \& Sampson D.D. (2014) Optical coherence tomography can assess skeletal muscle tissue from mouse models of muscular dystrophy by parametric imaging of the attenuation coefficient.

Biomedical Optics Express 5, 1217-32. 
Klyen B.R., Shavlakadze T., Radley-Crabb H.G., Grounds M.D. \& Sampson D.D. (2011) Identification of muscle necrosis in the mdx mouse model of Duchenne muscular dystrophy using three-dimensional optical coherence tomography. Journal of Biomedical Optics 16.

Lee J.I.H. \& Xia Y. (2013) Quantitative zonal differentiation of articular cartilage by microscopic magnetic resonance imaging, polarized light microscopy, and Fourier-transform infrared imaging. Microscopy Research and Technique 76, 625-32.

Lee W.N., Pernot M., Couade M., Messas E., Bruneval P., Bel A., Hagège A.A., Fink M. \& Tanter M. (2012) Mapping myocardial fiber orientation using echocardiography-based shear wave imaging. IEEE Transactions on Medical Imaging 31, 554-62.

LeGrice I.J., Smaill B.H., Chai L.Z., Edgar S.G., Gavin J.B. \& Hunter P.J. (1995a) Laminar structure of the heart: Ventricular myocyte arrangement and connective tissue architecture in the dog. American Journal of Physiology - Heart and Circulatory Physiology 269, H571-H82.

LeGrice I.J., Takayama Y. \& Covell J.W. (1995b) Transverse shear along myocardial cleavage planes provides a mechanism for normal systolic wall thickening. Circulation Research 77, 182-93.

Li X., Ranasinghesagara J.C. \& Yao G. (2008) Polarization-sensitive reflectance imaging in skeletal muscle. Optics Express 16, 9927-35.

Liu C.J., Black A.J., Wang H. \& Akkin T. (2016) Quantifying three-dimensional optic axis using polarization-sensitive optical coherence tomography. Journal of Biomedical Optics 21, 070501-.

Lovering R.M., Shah S.B., Pratt S.J.P., Gong W. \& Chen Y. (2013) Architecture of healthy and dystrophic muscles detected by optical coherence tomography. Muscle and Nerve 47, 588-90.

Lu Z., Kasaragod D. \& Matcher S.J. (2014) Conical scan polarization-sensitive optical coherence tomography. Biomedical Optics Express 5, 752-62.

Makita S., Yamanari M. \& Yasuno Y. (2010) Generalized Jones matrix optical coherence tomography: Performance and local birefringence imaging. Optics Express 18, 854-76. 
McMillan A.B., Shi D., Pratt S.J.P. \& Lovering R.M. (2011) Diffusion tensor MRI to assess damage in healthy and dystrophic skeletal muscle after lengthening contractions. Journal of Biomedicine and Biotechnology 2011.

Mekkaoui C., Chen I.Y., Chen H.H., Kostis W.J., Pereira F., Jackowski M.P. \& Sosnovik D.E. (2015) Differential response of the left and right ventricles to pressure overload revealed with diffusion tensor MRI tractography of the heart in vivo. Journal of Cardiovascular Magnetic Resonance 17, 1-2.

Mekkaoui C., Huang S., Chen H.H., Dai G., Reese T.G., Kostis W.J., Thiagalingam A., Maurovich-Horvat P., Ruskin J.N., Hoffmann U., Jackowski M.P. \& Sosnovik D.E. (2012) Fiber architecture in remodeled myocardium revealed with a quantitative diffusion CMR tractography framework and histological validation. Journal of Cardiovascular Magnetic Resonance 14.

Mercuri E. \& Muntoni F. (2013) Muscular dystrophy: New challenges and review of the current clinical trials. Current Opinion in Pediatrics 25, 701-7.

Meyers T.A. \& Townsend D. (2015) Early right ventricular fibrosis and reduction in biventricular cardiac reserve in the dystrophin-deficient mdx heart. American Journal of Physiology - Heart and Circulatory Physiology 308, H303-H15.

Mittelstaedt D., Xia Y., Shmelyov A., Casciani N. \& Bidthanapally A. (2011) Quantitative determination of morphological and territorial structures of articular cartilage from both perpendicular and parallel sections by polarized light microscopy. Connective Tissue Research 52, 512-22.

Niederer P.F., Lunkenheimer P.P. \& Cryer C.W. (2004) On the significance of fiber branching in the human myocardium. Biomechanics and Modeling in Mechanobiology 3, 1-5.

Nielles-Vallespin S., Mekkaoui C., Gatehouse P., Reese T.G., Keegan J., Ferreira P.F., Collins S., Speier P., Feiweier T., De Silva R., Jackowski M.P., Pennell D.J., Sosnovik D.E. \& Firmin D. (2013) In vivo diffusion tensor MRI of the human heart: Reproducibility of breath-hold and navigator-based approaches. Magnetic Resonance in Medicine 70, 454-65.

Nigro G., Comi L.I., Politano L. \& Bain R.J.I. (1990) The incidence and evolution of cardiomyopathy in Duchenne muscular dystrophy. International Journal of Cardiology 26, 271-7. 
Ortiz S., Siedlecki D., Grulkowski I., Remon L., Pascual D., Wojtkowski M. \& Marcos S. (2010) Optical distortion correction in Optical Coherence Tomography for quantitative ocular anterior segment by three-dimensional imaging. Optics Express 18, 2782-96.

Papadacci C., Tanter M., Pernot M. \& Fink M. (2014) Ultrasound backscatter tensor imaging (BTI): Analysis of the spatial coherence of ultrasonic speckle in anisotropic soft tissues. IEEE Transactions on Ultrasonics, Ferroelectrics, and Frequency Control 61, 986-96.

Park B.H., Pierce M.C., Cense B. \& De Boer J.F. (2004) Jones matrix analysis for a polarization-sensitive optical coherence tomography system using fiber-optic components. Optics Letters 29, 2512-4.

Pasquesi J.J., Schlachter S.C., Boppart M.D., Chaney E., Kaufman S.J. \& Boppart S.A. (2006) In vivo detection of exercise-induced ultrastructural changes in genetically-altered murine skeletal muscle using polarization-sensitive optical coherence tomography. Optics Express 14, 1547-56.

Pirolo J.S., Hutchins G.M., Moore G.W. \& Weisfeldt M.L. (1982) Myocyte disarray develops in papillary muscles released from normal tension after mitral valve replacement. Circulation 66, 841-6.

Ponrartana S., Ramos-Platt L., Wren T., Hu H., Perkins T., Chia J. \& Gilsanz V. (2014) Effectiveness of diffusion tensor imaging in assessing disease severity in Duchenne muscular dystrophy: preliminary study. Pediatric Radiology, 1-8.

Radley H.G., Davies M.J. \& Grounds M.D. (2008) Reduced muscle necrosis and longterm benefits in dystrophic mdx mice after cV1q (blockade of TNF) treatment. Neuromuscular Disorders 18, 227-38.

Radley H.G., De Luca A., Lynch G.S. \& Grounds M.D. (2007) Duchenne muscular dystrophy: Focus on pharmaceutical and nutritional interventions. International Journal of Biochemistry and Cell Biology 39, 469-77.

Rohde G.K., Aldroubi A. \& Dawant B.M. (2003) The adaptive bases algorithm for intensity-based nonrigid image registration. IEEE Transactions on Medical Imaging 22, 1470-9. 
Ryan J.J. \& Archer S.L. (2014) The Right Ventricle in Pulmonary Arterial Hypertension: Disorders of metabolism, angiogenesis and adrenergic signaling in right ventricular failure. Circulation Research 115, 176-88.

Sasaoka T., Imamura M., Araishi K., Noguchi S., Mizuno Y., Takagoshi N., Hama H., Wakabayashi-Takai E., Yoshimoto-Matsuda Y., Nonaka I., Kaneko K., Yoshida M. \& Ozawa E. (2003) Pathological analysis of muscle hypertrophy and degeneration in muscular dystrophy in $\gamma$-sarcoglycan-deficient mice.

Neuromuscular Disorders 13, 193-206.

Saupe N., White L.M., Stainsby J., Tomlinson G. \& Sussman M.S. (2009) Diffusion tensor imaging and fiber tractography of skeletal muscle: Optimization of b value for imaging at 1.5 T. American Journal of Roentgenology 192, W282-W90.

Scolaro L., McLaughlin R.A., Klyen B.R., Wood B.A., Robbins P.D., Saunders C.M., Jacques S.L. \& Sampson D.D. (2012) Parametric imaging of the local attenuation coefficient in human axillary lymph nodes assessed using optical coherence tomography. Biomedical Optics Express 3, 366-79.

Shim V.B., Besier T.F., Lloyd D.G., Mithraratne K. \& Fernandez J.F. (2016) The influence and biomechanical role of cartilage split line pattern on tibiofemoral cartilage stress distribution during the stance phase of gait. Biomechanics and Modeling in Mechanobiology 15, 195-204.

Shin J.H., Bostick B., Fine D.M., Yue Y. \& Duan D. (2010) Duchenne cardiomyopathy gene therapy. In: Muscle Gene Therapy (pp. 141-62.

Shin J.H., Hakim C.H., Zhang K. \& Duan D. (2011) Genotyping mdx, mdx3cv, and $\mathrm{mdx} 4 \mathrm{cv}$ mice by primer competition polymerase chain reaction. Muscle and Nerve 43, 283-6.

Sicinski P., Geng Y., Ryder-Cook A., Barnard E., Darlison M. \& Barnard P. (1989) The molecular basis of muscular dystrophy in the mdx mouse: a point mutation. Science 244, 1578-80.

Sosnovik D.E., Mekkaoui C., Huang S., Chen H.H., Dai G., Stoeck C.T., Ngoy S., Guan J., Wang R., Kostis W.J., Jackowski M.P., Wedeen V.J., Kozerke S. \& Liao R. (2014) Microstructural Impact of Ischemia and Bone Marrow-Derived Cell Therapy Revealed With Diffusion Tensor Magnetic Resonance Imaging Tractography of the Heart In VivoCLINICAL PERSPECTIVE. Circulation 129, 1731-41. 
Sosnovik D.E., Wang R., Dai G., Reese T.G. \& Wedeen V.J. (2009a) Diffusion MR tractography of the heart. Journal of Cardiovascular Magnetic Resonance 11.

Sosnovik D.E., Wang R., Dai G., Wang T., Aikawa E., Novikov M., Rosenzweig A., Gilbert R.J. \& Wedeen V.J. (2009b) Diffusion spectrum mri tractography reveals the presence of a complex network of residual myofibers in infarcted myocardium. Circulation: Cardiovascular Imaging 2, 206-12.

Spurney C.F. (2011) Cardiomyopathy of duchenne muscular dystrophy: Current understanding and future directions. Muscle and Nerve 44, 8-19.

Streeter D.D. \& Bassett D.L. (1966) An engineering analysis of myocardial fiber orientation in pig's left ventricle in systole. The Anatomical Record 155, 503-11.

Streeter Jr D.D., Spotnitz H.M., Patel D.P., Ross Jr J. \& Sonnenblick E.H. (1969) Fiber orientation in the canine left ventricle during diastole and systole. Circulation research 24, 339-47.

Strijkers G.J., Bouts A., Blankesteijn W.M., Peeters T.H.J.M., Vilanova A., van Prooijen M.C., Sanders H.M.H.F., Heijman E. \& Nicolay K. (2009) Diffusion tensor imaging of left ventricular remodeling in response to myocardial infarction in the mouse. NMR in Biomedicine 22, 182-90.

Stuckey D.J., Carr C.A., Camelliti P., Tyler D.J., Davies K.E. \& Clarke K. (2012) In vivo MRI Characterization of Progressive Cardiac Dysfunction in the mdx Mouse Model of Muscular Dystrophy. PLoS ONE 7, e28569.

Taccardi B., Macchi E., Lux R.L., Ershler P.R., Spaggiari S., Baruffi S. \& Vyhmeister Y. (1994) Effect of myocardial fiber direction on epicardial potentials. Circulation 90, 3076-90.

Taylor E.N., Hoffman M.P., Barefield D.Y., Aninwene G.E., Abrishamchi A.D., Lynch T.L., Govindan S., Osinska H., Robbins J., Sadayappan S. \& Gilbert R.J. (2016) Alterations in Multi-Scale Cardiac Architecture in Association With Phosphorylation of Myosin Binding Protein-C. Journal of the American Heart Association: Cardiovascular and Cerebrovascular Disease 5, e002836.

Todorović M., Jiao S., Ai J., Pereda-Cubián D., Stoica G. \& Wang L.V. (2008) In vivo burn imaging using Mueller optical coherence tomography. Optics Express 16, 10279-84. 
Todorović M., Jiao S., Wang L.V. \& Stoica G. (2004) Determination of local polarization properties of biological samples in the presence of diattenuation by use of Mueller optical coherence tomography. Optics Letters 29, 2402-4.

Tseng W.Y.I., Dou J., Reese T.G. \& Van Wedeen J. (2006) Imaging myocardial fiber disarray and intramural strain hypokinesis in hypertrophic cardiomyopathy with MRI. Journal of Magnetic Resonance Imaging 23, 1-8.

Ugryumova N., Gangnus S.V. \& Matcher S.J. (2006) Three-dimensional optic axis determination using variable-incidence-angle polarization-optical coherence tomography. Optics Letters 31, 2305-7.

Ugryumova N., Jacobs J., Bonesi M. \& Matcher S.J. (2009) Novel optical imaging technique to determine the 3-D orientation of collagen fibers in cartilage: variable-incidence angle polarization-sensitive optical coherence tomography. Osteoarthritis and Cartilage 17, 33-42.

Valentine B.A., Cummings J.F. \& Cooper B.J. (1989) Development of Duchenne-type cardiomyopathy. Morphologic studies in a canine model. The American Journal of Pathology 135, 671-8.

Van Donkelaar C.C., Kretzers L.J.G., Bovendeerd P.H.M., Lataster L.M.A., Nicolay K., Janssen J.D. \& Drost M.R. (1999) Diffusion tensor imaging in biomechanical studies of skeletal muscle function. Journal of Anatomy 194, 79-88.

Vetter F.J. \& McCulloch A.D. (1998) Three-dimensional analysis of regional cardiac function: A model of rabbit ventricular anatomy. Progress in Biophysics and Molecular Biology 69, 157-83.

Vetter F.J., Simons S.B., Mironov S., Hyatt C.J. \& Pertsov A.M. (2005) Epicardial fiber organization in swine right ventricle and its impact on propagation. Circulation Research 96, 244-51.

Wallenburg M.A., Wood M.F.G., Ghosh N. \& Vitkin I.A. (2010) Polarimetry-based method to extract geometry-independent metrics of tissue anisotropy. Optics Letters 35, 2570-2.

Wang H., Lenglet C. \& Akkin T. (2015a) Structure tensor analysis of serial optical coherence scanner images for mapping fiber orientations and tractography in the brain. Journal of Biomedical Optics 20. 
Wang T.T., Kwon H.S., Dai G., Wang R., Mijailovich S.M., Moss R.L., So P.T.C., Wedeen V.J. \& Gilbert R.J. (2010) Resolving myoarchitectural disarray in the mouse ventricular wall with diffusion spectrum magnetic resonance imaging. Annals of Biomedical Engineering 38, 2841-50.

Wang Y., Ravanfar M., Zhang K., Duan D. \& Yao G. (2016) Mapping 3D fiber orientation in tissue using dual-angle optical polarization tractography. Biomedical Optics Express 7, 3855-70.

Wang Y. \& Yao G. (2013) Optical tractography of the mouse heart using polarizationsensitive optical coherence tomography. Biomedical Optics Express 4, 2540-5.

Wang Y., Zhang K., Duan D. \& Yao G. (2017) Heart structural remodeling in a mouse model of Duchenne cardiomyopathy revealed using optical polarization tractography [Invited]. Biomedical Optics Express 8, 1271-6.

Wang Y., Zhang K., Wasala N.B., Duan D. \& Yao G. (2015b) Optical polarization tractography revealed significant fiber disarray in skeletal muscles of a mouse model for Duchenne muscular dystrophy. Biomedical Optics Express 6, 347-52.

Wang Y., Zhang K., Wasala N.B., Yao X., Duan D. \& Yao G. (2014) Histology validation of mapping depth-resolved cardiac fiber orientation in fresh mouse heart using optical polarization tractography. Biomedical Optics Express 5, 284355 .

Weber K.T. (1989) Cardiac interstitium in health and disease: The fibrillar collagen network. Journal of the American College of Cardiology 13, 1637-52.

Xia Y., Moody J.B., Burton-Wurster N. \& Lust G. (2001) Quantitative in situ correlation between microscopic MRI and polarized light microscopy studies of articular cartilage. Osteoarthritis and Cartilage 9, 393-406.

Yang X., Chin L., Klyen B.R., Shavlakadze T., McLaughlin R.A., Grounds M.D. \& Sampson D.D. (2013) Quantitative assessment of muscle damage in the mdx mouse model of Duchenne muscular dystrophy using polarization-sensitive optical coherence tomography. Journal of Applied Physiology 115, 1393-401.

Yang X., Lorenser D., McLaughlin R.A., Kirk R.W., Edmond M., Simpson M.C., Grounds M.D. \& Sampson D.D. (2014) Imaging deep skeletal muscle structure using a high-sensitivity ultrathin side-viewing optical coherence tomography needle probe. Biomedical Optics Express 5, 136-48. 
Zaidman C.M., Connolly A.M., Malkus E.C., Florence J.M. \& Pestronk A. (2010) Quantitative ultrasound using backscatter analysis in Duchenne and Becker muscular dystrophy. Neuromuscular Disorders 20, 805-9.

Zhang J., Richards L.J., Yarowsky P., Huang H., Van Zijl P.C.M. \& Mori S. (2003) Three-dimensional anatomical characterization of the developing mouse brain by diffusion tensor microimaging. NeuroImage 20, 1639-48.

Zhao M., Kuo A.N. \& Izatt J.A. (2010) 3D refraction correction and extraction of clinical parameters from spectral domain optical coherence tomography of the cornea. Optics Express 18, 8923-36. 


\section{VITA}

Yuanbo Wang was born in Hebei, China. He received his B.E. degree in Telecommunication Engineering in 2004 from Hebei University, Baoding, China. He also received an M.S. degree in Telecommunication \& Information System in 2005 from University of Essex, Colchester, UK; and an M.S. degree in Photonics in 2010 from Boston University, Boston, USA. He started his PhD program in Bioengineering in August 2012 at University of Missouri. His research interest is biomedical optical imaging. 